\title{
Measurement of the Helicity of $W$ Bosons in Top Quark Decays
}

\author{
by
}

\section{David J. Winn}
A dissertation submitted in partial fulfillment
of the requirements for the degree of
Doctor of Philosophy
(Physics)
in The University of Michigan
2000

Doctoral Committee:

\author{
Associate Professor Dante Amidei, Chair \\ Professor Timothy Chupp \\ Professor Martin Einhorn \\ Associate Professor Keith Riles \\ Professor Ralph Williams
}




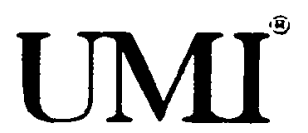

\section{UMI Microform9977286}

Copyright 2000 by Bell \& Howell Information and Leaming Company.

All rights reserved. This microform edition is protected against unauthorized copying under Title 17, United States Code.

Bell \& Howell Information and Learning Company

300 North Zeeb Road

P.O. Box 1346

Ann Arbor, MI 48106-1346 


\section{Table of Contents}

List of Tables $\ldots \ldots \ldots \ldots \ldots \ldots \ldots \ldots \ldots \ldots \ldots \ldots \ldots$

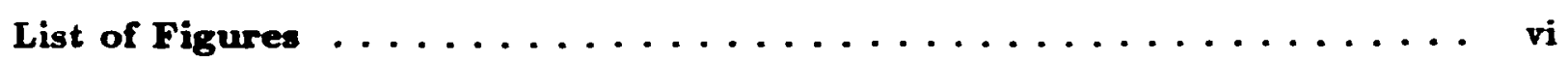

List of Appendices $\ldots \ldots \ldots \ldots \ldots \ldots \ldots \ldots \ldots \ldots \ldots$

Chapter

1. The Standard Model and the Top Quark .............. 1

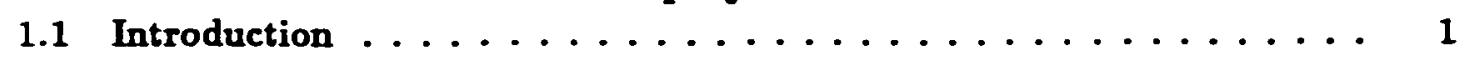

1.2 The Standard Model $\ldots \ldots \ldots \ldots \ldots \ldots \ldots$

1.2.1 Quantum Chromodynamics ............... 3

1.2.2 The Electroweak Force .................. 4

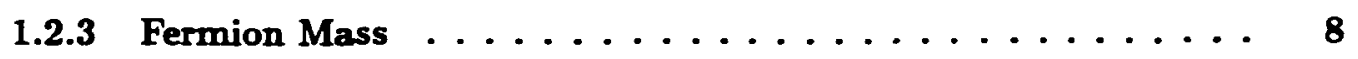

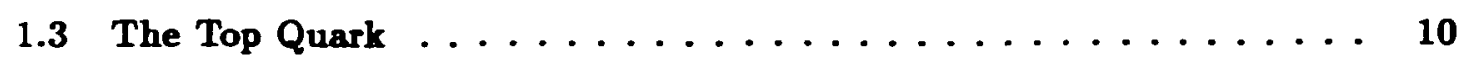

1.3.1 Identifying Top Quark Events ................. 11

1.4 Strategy for Measuring W Helicity . . . . . . . . . . . . . . . . 12

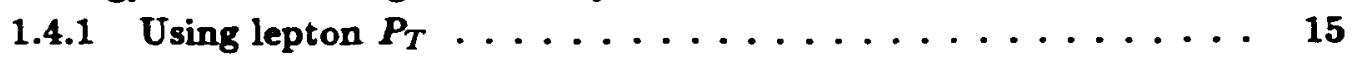

2. The Fermilab Tevatron and the CDF Detector $\ldots \ldots \ldots \ldots \ldots \ldots$

2.1 The Tevatron . . . . . . . . . . . . . . . . . . 17

2.2 The Collider Detector at Fermilab ................. 18

2.2.1 The Tracking Systems ................... 22

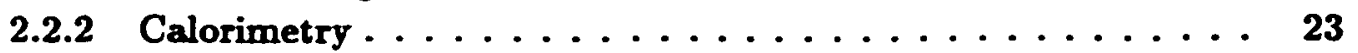

2.2.3 Muon Detectors ..................... 24

2.2.4 The CDF Trigger System ................. 26

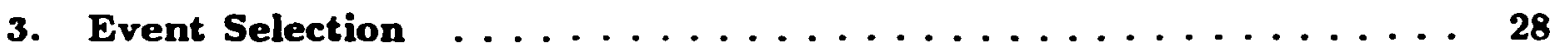

3.1 Inclusive High $P_{T}$ Electrons . . . . . . . . . . . . . . 29

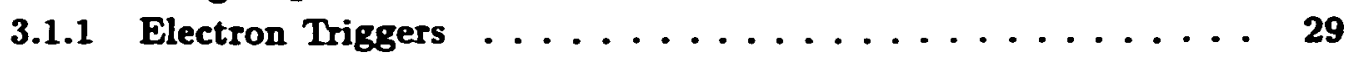

3.1.2 Offline Electron Selection . . . . . . . . . . . . . 29

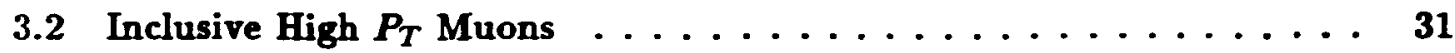

3.2.1 Muon Triggers ..................... 32

ii 
3.3 Offline Muon Selection ..................... 32

3.4 Lepton + Jet Event Selection . . . . . . . . . . . . . . 33

$3.4 .1 \quad Z$ removal . . . . . . . . . . . . . . . . . . 34

3.4.2 Silicon Vertex Tracker (SVX) Tagging Sample . . . . . . . . 35

3.4.3 Soft Lepton Tag (SLT) sample ............... 35

3.4 .4 No-tag Sample .................... 36

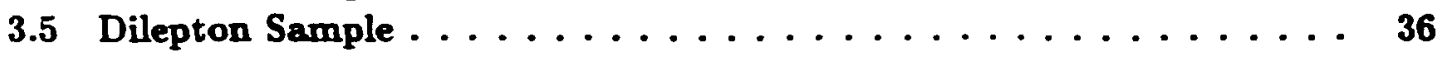

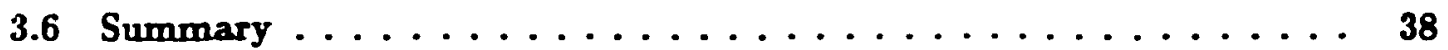

4. Models for Signal and Background . . . . . . . . . . . . 39

4.1 GGWIG: A Custom HERWIG Monte Carlo Generator . . . . . . . . . 41

4.2 PYTHIA ........................ 41

4.2.1 Background Models ...................42 42

5. Measuring the Helicity of the $\mathbf{w}$ Boson $\ldots \ldots \ldots \ldots \ldots$

5.1 Likelihood Function . . . . . . . . . . . . . . . . . 46

5.2 Verification of the Likelihood Technique . . . . . . . . . . . 48

5.2.1 Consistency and Sensitivity . . . . . . . . . . . 48

5.2.2 Investigation of the Background Fraction Term in the Likelihood 50

5.3 Acceptance Bias . . . . . . . . . . . . . . . . . 50

5.3.1 Acceptance Correction for Lepton + Jets . . . . . . . . . . 51

5.3 .2 Acceptance Correction for Dileptons . . . . . . . . . . . 53

5.3.3 Computing Acceptance Corrections with Pythia . . . . . . . 55

5.3.4 Combining the Corrections . . . . . . . . . . . . . 56

5.4 Measurement of $\mathcal{F}_{0} \ldots \ldots \ldots \ldots \ldots \ldots \ldots \ldots$

5.5 Measurement of $\mathcal{F}_{+} \ldots \ldots \ldots \ldots \ldots \ldots \ldots \ldots$

6. Systematic Uncertainties .................... 62

6.1 Top Mass Bias . . . . . . . . . . . . . . . . . . 63

6.1.1 Non-W Background Normalization . . . . . . . . . . 63

$6.2 \quad b$ Quark Tagging Efficiency .................... 66

6.3 Monte Carlo Statistics . . . . . . . . . . . . . . . . . . 67

6.4 Acceptance Uncertainties ..................... 68

6.5 Non-W Background Shape . . . . . . . . . . . . . . . . 69

6.6 Gluon Radiation ... . . . . . . . . . . . . . . . . . 75

6.7 Parton Distribution Functions . . . . . . . . . . . . 77

6.8 Total Systematic Uncertainty . . . . . . . . . . . . . . 78

7. Conclusions $\ldots \ldots \ldots \ldots \ldots \ldots \ldots \ldots \ldots \ldots \ldots \ldots \ldots \ldots \ldots$

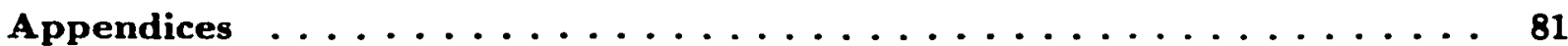

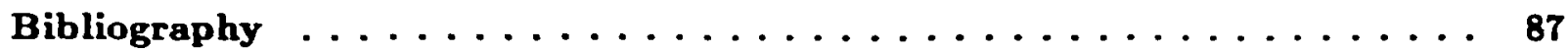

iii 


\section{List of Tables}

Table

1.1 Mass and charge of the gauge bosons in the standard model. . . . . . . . 8

1.2 Mass and charge of the fermions in the standard model. . . . . . . . . . . 9

2.1 Summary CDF calorimetry properties. The symbol $\oplus$ signifies addition in quadrature. Energy resolutions for the electromagnetic calorimeters are for incident electrons and photons; for the hadronic calorimeters, they are for incident isolated pions. Energy is in GeV. Thicknesses are given in radiation lengths $\left(X_{0}\right)$ for electromagnetic calorimeters, and in interaction lengths $\left(\lambda_{0}\right)$ for hadronic calorimeters. . . . . . . . . . . . . . . . . . .

3.1 We list here the differences between the loose and tight selection requirements for electrons. . . . . . . . . . . . . . . . . . . .

3.2 Observed events and predicted backgrounds for the lepton + jet data subsamples. The lepton + jet entry is the sum of the three entries which precede it.

4.1 A table of dilepton backgrounds . . . . . . . . . . . . .

5.1 Number of events which satisfy selection cuts in our acceptance calculation. The third column is reproduced from Ref . . . . . . . . . . . . . 53

5.2 Acceptances for the three helicity categories in dilepton events. . . . . . . . 54

5.3 Helicity dependent acceptances for Pythia Monte Carlo. Errors are statistical. 56

5.4 Fits to the data for the longitudinal W fraction using GGWIG templates. . 60

5.5 The table entries contain the right-handed fraction returned from fits to the data with various mixes of non-isolated leptons in the background model.

6.1 Preliminary estimate of changes to the backgrounds when the new $b$ tag

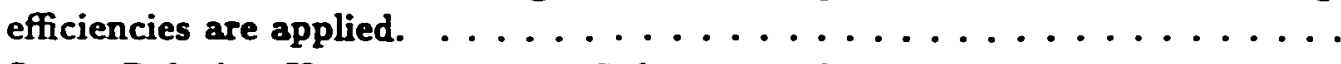

6.2 Some Relative Uncertainties on Dilepton and Lepton + Jet acceptances. Only acceptance uncertainties which are uncorrelated to any shape uncertainties are listed in this table. These uncertainties are taken from Ref. . . . 
6.3 Fit results for the longitudinal fraction using several different non-W background models. One-half the range of the results which use Pythia Monte Cario as a non-W background model is quoted as the non-W background shape uncertainty. ....................... 72

6.4 Table of uncertainties . . . . . . . . . . . . . . . . 78 


\section{List of Figures}

Figure

1.1 Lepton $\boldsymbol{P}_{T}$ distributions for the three $W$ helicities. The solid circles are from negative helicity $W^{+}$and positive helicity $W^{-}$, the open circles are from longitudinal $W^{+}$and $W^{-}$, and the closed squares are from positive helicity $W^{+}$and negative helicity $W^{-}$. All three distributions are normalized to the same area. . . . . . . . . . . . . . . . . . . . 16

2.1 A schematic diagram of the Tevatron collider complex. . . . . . . . . . . . 19

2.2 A schematic diagram of the CDF detector plant. Note that CDF has a cylindrical symmetry and that this picture shows only a quarter of the detector. For the entire detector rotate this picture about the beamline and add its mirror image scross the left side of the picture. . . . . . . . . . . .

3.1 The lepton $P_{T}$ distributions for the $1+$ jet and dilepton samples. We shall extract the longitudinal helicity fraction from these distributions by fitting them to negative helicity and longitudinal models using a maximum loglikelihood technique. .....................

4.1 Comparison between lepton $P_{T}$ distributions from GGWIG set to $70 \%$ longitudinal $W$ compostion and HERWIG. In both samples the top mass is $\mathbf{1 7 5}$

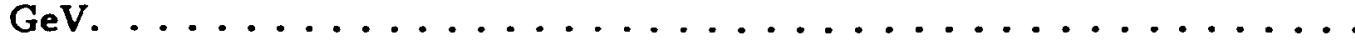

4.2 Lepton $P_{T}$ distribution from VECBOS with $\mathrm{W}+3$ Jet matrix elements compared with lepton + jet data from run $\mathrm{I} . \ldots \ldots \ldots \ldots$

5.1 The circles in this plot represent the $P_{T}$ distributions for leptons from negative helicity and longitudinal $W$ Bosons, as predicted by GGWIG. The smooth lines are the best fit to the Monte Carlo distributions using the product of an exponential and a polynomial function. . . . . . . . . . . .

5.2 Pseudo-experiment study of the maximum likelihood procedure. Top plot is distribution of returned values for input $\mathcal{F}_{0}=0.70$, bottom plot is the distribution of the pulls. .................... 49

5.3 Checks of the sensitivity to the background assumptions. . . . . . . . 51 
5.4 Here we show the acceptance corrections for both the lepton +jets and dilepton samples as a function of $\mathcal{F}_{0}^{\text {fit }}$. We do not show the combined correction because it is so close to the lepton + jet correction that they cannot be distinguished from one another on the scale of this figure. . . . . . . . . .

5.5 Lepton $P_{T}$ distributions for the lepton +jet and dilepton subsamples. The lepton + jet subsamples are added together to simplify presentation. The data (points) are compared with the result of the combined fit (solid line) and with the background component of the fit (dashed line). . . . . . . .

5.6 Results of a pseudo-experiment study of the sensitivity of the positive helicity measurement. The width of the Gaussian distribution is only 0.15 , but when compared to the width of the physically allowable region $(0.0$ to 0.30$)$ it appears that we have very little sensitivity. . . . . . . . . . . .

6.1 Comparison of negative helicity and longitudinal templates at different top mass values. . . . . . . . . . . . . . . . . . . .

6.2 Measured helicity fraction versus the mass of top which is used in our analysis templates. The uncertainties include only the uncertainty on the Monte Carlo template because all other uncertainties in these measurements are highly

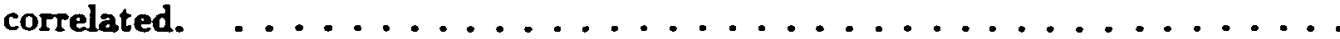

6.3 Lepton $P_{T}$ spectrum in our PYTHIA $b \bar{b}$ sample as a function of $\mathscr{H}_{T}$ and lepton isolation. In region $A F_{T}<15$ and $I<0.1$, in regien $B E_{T}<15$ and $I>0.2$, in region $\mathrm{C} \mathbb{F}_{T}>20$ and $I>0.2$, and finally, in region $\mathrm{D} \boldsymbol{F}_{T}>20$ and $I<0.1$.

6.4 Comparison of Pythia $b \bar{b}$ Monte Carlo to Run $\mathrm{Ib}$ data in several kinematic variables. The events in these plots come from region $B$.

6.5 Comparison of Pythia $b \bar{b}$ Monte Carlo to Run Ib data in several kinematic variables. The events in these plots come from region $\mathrm{C} . \quad \ldots \ldots \ldots \ldots$

6.6 Comparison of the Pythia lepton $P_{T}$ spectrum with radiation on to the Pythia lepton $\boldsymbol{P}_{T}$ spectrum with initial or final state radiation turned off. . . . . . . 


\section{List of Appendices}

\section{Appendix}

A. The CDF Collaboration $\ldots \ldots \ldots \ldots \ldots \ldots \ldots \ldots$

viii 


\section{Chapter 1}

\section{The Standard Model and the Top Quark}

\section{$1.1 \quad$ Introduction}

This thesis describes a measurement of the decay properties of the top quark ${ }^{1}$. The six quarks are fundamental building blocks matter in the universe. The most common quarks, named up and down, combine to form the protons and neutrons which exist at the nucleus of all atoms. Together with electrons they make up all matter that can be touched or seen with human senses. The top quark, which was discovered recently by the CDF and D0 collaborations $[2,3]$, is the most massive of the six quarks. The decay properties of all the quarks are governed by the weak interaction of the standard model of particle physics, so the enormous mass of the top quark provides us with an opportunity to study the weak interaction at a much higher mass scale than was previously available.

In the remainder of this chapter we describe the standard model [4]. Our focus will be the weak sector of the standard model and its predictions about the helicity of $W$ bosons in top quark decays. In chapter 2 we describe the CDF detector apparatus. Event selection is described in chapter 3 , and we describe the models for the signals and the backgrounds that are used in this analysis in chapter 4 . The methods used to measure the helicity of the $W$ boson are outlined in chapter 5 , and the systematic uncertainties in the measurement are discussed in chapter 6.

\footnotetext{
${ }^{1}$ This measurement was first published in [1].
} 


\subsection{The Standard Model}

The standard model of high energy physics is a theory which successfully describes the interactions of all fundamental particles which have been observed to date. The building blocks of the theory are the quarks and leptons. These particles are spin-1/2 fermions. Each generation contains two leptons and two quarks, for a total of six quarks and six leptons in the three generations. The quarks and leptons interact via four forces: electromagnetism, the weak force, the strong force, and gravity. According to the standard model they also interact via a fifth force associated with the origin of their masses ${ }^{2}$.

Quarks are fermions that interact with the strong force. There are two types of quark. $U_{p}$ type quarks possess an electrical charge of $2 / 3$, and down type quarks possess an electrical charge of $-1 / 3$. The up type quarks are up $(u)$, charm $(c)$, and top $(t)$ and the down type quarks are down (d), strange (s), and bottom (b).

Leptons are fermions which interact with the weak force and the electromagnetic force. Leptons come in two types, charged leptons and neutrinos. The charged leptons have an electrical charge of -1 and are named the electron (e), the muon ( $\mu$ ), and the tau $(\tau)$. The neutrinos have 0 electrical charge, and interact with other particles only via the weak force. The neutrinos are named for their weak partner. Thus we have the electron neutrino $\left(\nu_{e}\right)$, the muon neutrino $\left(\nu_{\mu}\right)$, and the tau neutrino $\left(\nu_{\tau}\right)$. Note that all neutrinos (anti-neutrinos) are left (right) handed ${ }^{3}$.

The photon $(\gamma)$ is a massless spin-1 gauge boson that mediates the electromagnetic force. The weak force is mediated by the $W$ and $Z$, which are both massive spin-1 gauge bosons. The strong force is mediated by eight spin-1 gluons $(g)$, which are massless. The gravitational force is so weak compared to the other three that we have not been able to observe the gauge boson that mediates it. If it exists it would be a massless spin-2 boson called a graviton.

The theories which describe the interactions between the fermions and the gauge

\footnotetext{
${ }^{2}$ This force is called the Higgs force. The standard model description of this force requires the existence of the Higgs boson, which has not yet been discovered

${ }^{3}$ The handedness, or chirality, property of the fermions is discussed in Section 1.2.2
} 
bosons are local gauge theories. They are so called because the Lagrangian function which summarizes the interactions is invariant under an appropriate local gauge transformation. For instance, quantum electrodynamics (QED), the theory of electromagnetic interactions, is invariant under the following local gauge transformation:

$$
\begin{aligned}
\psi(x) & \rightarrow \exp [\operatorname{ie\Lambda }(x)] \psi(x) \\
A_{\mu}(x) & \rightarrow A_{\mu}(x)+\partial \Lambda(x) / \partial x_{\mu}
\end{aligned}
$$

where $\psi(x)$ is the fermion field, $A_{\mu}(x)$ is the photon field, $e$ is the strength of the photonfermion coupling, and $\Lambda(x)$ is any arbitrary differentiable function of $x$. The phase factor $\exp [i e \Lambda(x)]$ belongs to the symmetry group $U(1)$ of unitary transformations in one dimension. The importance of gauge invariance is difficult to overstate. If we postulate that the fermion field is locally invariant under a U(1) gauge transformation then the existence of the photon field is mandatory, and the nature of its coupling to the fermion field is completely specified. Note that the photon must be massless in order for the theory to be exactly $U(1)$ invariant. The strong and weak forces are also described by local gauge theories.

\subsubsection{Quantum Chromodynamics}

The portion of the standard model that describes the strong force is referred to as quantum chromodynamics (QCD). In QCD the quark fields possess a color charge which can have three different values: blue, yellow, and red ${ }^{4}$ The three quark color fields form a triplet field $\psi=\left(\psi_{r}, \psi_{g}, \psi_{b}\right)$ which is invariant under the SU(3) group of local gauge transformations. This local gauge invariance requires the existence of eight massless gauge boson fields. These are the gluons mentioned above. Each of the gluon fields carries a double color charge.

A subtle consequence of the fact that the gluons carry the charge of the interactions that they mediate is that the force between two color charges increases as the distance

\footnotetext{
${ }^{1}$ The color charge is so named because its additive properties are similar to the visible spectrum of light. Red, yellow, and blue color charges sum to sero when present in equal measure, just as red, yellow, and blue light become white light when mired together.
} 
between them increases. This is in direct contrast to the electromagnetic force, which decreases as a function of the distance between two charges. This property of the strong force implies that color charges cannot freely exist, because the energy used to separate two or more color charged particles will in general create new color charged particles that will bind with the original particles to form new color neutral composites. This is called color confinement. A second consequence of gluon color charge is that the color charge carried by a quark is diffused outward by the virtual gluon field which surrounds it. This means that QCD interactions at short range have a much smaller coupling than QCD interactions at long range. This property is called asymptotic freedom.

QCD interactions are very complicated. An interaction which creates a quark will inevitably be followed by a flurry of QCD activity called "hadronization" wherein the quark is confined within a color neutral hadron. In general, this process produces many hadrons in addition to the one that contains the quark produced by the original interaction, and the energy and momentum of the original quark can only be measured accurately if all of the hadrons that are produced can be observed, and that is a very difficult endeavor. Thus, it is often more convenient to focus on measurements that involve the colorless leptons, which can be observed directly.

\subsubsection{The Electroweak Force}

The electromagnetic and weak forces are described by a single local gauge theory, called the electroweak theory. The gauge group of the electroweak theory is $S U(2)_{L} \times U(1)$. The subscript $L$ refers to the chirality of the fermions that the gauge transformation operates on. Chirality is a property which is related to the spin and helicity of the fermion:

Helicity Helicity is the projection of a particle's spin along its direction of motion. Because spin is quantized, there are a limited number of helicity values that a particle can possess. The number of possible spin projections is $2 j+1$, where $j$ is the spin of the particle, and the values range from $-1 j$ to $+1 j$ in integer increments. Thus a spin- $\frac{1}{2}$ particle can have a helicity value of $-\frac{1}{2}$ or $\frac{1}{2}$ and a spin-1 particle can have a helicity 
value of $-1,0$, or 1 . Note that throughout this document we will often refer to the 0 helicity state of a vector boson as its "longitudinal" mode. This nomenclature derives from the fact that the polarization vector of the boson points along its direction of motion when it has 0 helicity. For massive particles helicity is not Lorentz invariant, so the frame in which it is measured must be specified.

Chirality An important basis set for the fermion spinors is called the chiral basis. For massless fermions the chiral spinors correspond to the $+\frac{1}{2}$ and $-\frac{1}{2}$ helicity states for the fermion and the anti-fermion. The $+\frac{1}{2}$ state is called a right-handed chiral state and is denoted by the subseript $R$. Similarly, the $-\frac{1}{2}$ state is called a left-handed chiral state and is denoted by the subscript $L$. For massive fermions the right and left handed chiral states are orthogonal mixtures of the positive and negative helicity states. Chirality is important in the theory of electroweak interactions, because the left chiral fields transform as a doublet under an electroweak gauge rotation whereas the right chiral fields transform as a singlet.

Under the U(1) hypercharge gauge group each fermion field is multiplied by a phase factor, analogous to the electromagnetic phase factor described above. Under the $\mathrm{SU}(2)_{L}$ portion of the gauge group, fermion fields transform as doublets or singlets as follows:

Quarks $\left[\begin{array}{l}u \\ d\end{array}\right]_{L},\left[\begin{array}{l}c \\ s\end{array}\right]_{L},\left[\begin{array}{l}t \\ b\end{array}\right]_{L}, u_{R}, d_{R}, c_{R}, s_{R}, t_{R}, b_{R}$.

Leptons $\left[\begin{array}{c}e \\ \nu_{e}\end{array}\right]_{L},\left[\begin{array}{c}\mu \\ \nu_{\mu}\end{array}\right]_{L},\left[\begin{array}{c}\tau \\ \nu_{\tau}\end{array}\right]_{L}, e_{R}, \mu_{R}, \tau_{R}$.

The pairs of left-handed fermions are called weak isospin doublets. The right-handed ( $R$ ) particles are weak isospin singlets, and are unaffected by $S U(2)_{L}$ transformations. The weak isospin doublets transform as:

$$
\left(\psi_{L}^{\dagger}, \psi_{L}^{\frac{1}{L}}\right) \rightarrow \exp [i g \Lambda(x)]\left(\psi_{L}^{\dagger}, \psi_{L}^{\frac{1}{}}\right)
$$


The $\uparrow$ and $\downarrow$ refer to the upper and lower fermion in the parentheses above, and $\Lambda(x)$ is a $2 \times 2$ matrix which operates on the fermion doublet. Note that the off-diagonal elements of $\Lambda$ can transform one of the fermions in the doublet into the other fermion in the doublet.

Four gauge boson fields are required to maintain electroweak gauge invariance. These fields have been observed experimentally, but three of them (the $W^{+}, W^{-}$, and the $Z$ ) are massive. This means that electroweak symmetry is broken in nature.

The unbroken gauge group requires four massless gauge bosons, three for the $S U(2)_{L}$ gauge group and one for the U(1) gauge group. For convenience, we will call the three gauge bosons of the $\mathrm{SU}(2)_{L}$ group $W_{1}, W_{2}$, and $W_{3}$, and the gauge bosons of the U(1) group $B$. Note that the electroweak gauge fields that have been observed experimentally are a linear combination of these four fields. The gauge bosons have a spin of 1 . However, they have only 2 spin degrees of freedom, because massless vector bosons are forbidden from having a longitudinal polarization.

The exact mechanism whereby the electroweak gauge symmetry is broken has not yet been established experimentally. The standard model method for breaking the symmetry is called the Higgs mechanism. In the Higgs mechanism, a complex scalar doublet $\Phi=\left(\phi^{+}, \phi^{0}\right)$ is introduced. This scalar field is named the Higgs doublet, and it is invariant under $\mathrm{SU}(2)_{L}$ gauge transformations. This doublet has electroweak quantum numbers, and thus interacts with the bosons of the electroweak gauge group. The Higgs doublet also has a self interaction, with potential:

$$
V=\mu^{2}|\Phi|^{2}+\lambda|\Phi|^{4}
$$

where $|\Phi|^{2}=\left(\phi^{+}\right)^{2}+\left(\phi^{0}\right)^{2}$. For $\mu^{2}<0$ the ground state of $|\Phi|^{2}$ occurs at $|\Phi|^{2}=-\frac{1}{2} \mu^{2} / \lambda$. In other words, the theory has a non-zero vacuum expectation value (vev).

It is inconvenient to work with a field that has a non-zero vacuum expectation value, so the Higgs doublet is redefined as follows:

$$
\Phi(x)=\exp \left(\frac{i \xi(x) \cdot \tau}{2 v}\right)\left(\begin{array}{c}
0 \\
(v+H(x)) / \sqrt{2}
\end{array}\right),
$$


where $v$ is the modulus of the vev $\left(v / \sqrt{2}=\left(-\mu^{2} / 2 \lambda\right)^{\frac{1}{2}}\right.$, the $\xi$ are three scalar fields called Goldstone bosons, the $\tau$ are the Pauli spin matrices, and $\boldsymbol{H}(\boldsymbol{x})$ is a scalar field called the Higgs boson. All of the scalar fields have zero vev in this formulation. Note that the Goldstone bosons can be removed by a finite gauge transformation. The gauge in which they appear to vanish is called the unitary gauge. The degrees of freedom represented by the Goldstone bosons are absorbed into the $W$ and $Z$ bosons, which thereby acquire a longitudinal degree of freedom and a mass.

We now redefine the gauge bosons of the theory as follows:

$$
\begin{aligned}
W^{+} & =W^{1}-i W^{2} \\
W^{-} & =W^{1}+i W^{2} \\
Z & =\sin \theta_{w} B+\cos \theta_{w} W^{3} \\
A & =\cos \theta_{w} B-\sin \theta_{w} W^{3}
\end{aligned}
$$

The parameter $\theta_{w}$ is called the electroweak mixing angle, and its value must be determined experimentally. $A$ is the photon of QED. Note that the linear combinations that compose the $A$ and the $Z$ were chosen to guarantee that the neutrino does not interact with the photon $(A)$. The $W^{+}$and $W^{-}$interactions violate parity maximally, because they do not interact with right-handed fermions (or left-handed anti-fermions). The $Z$ boson does interact with both left-handed and right-handed fermions, but the interaction is not symmetric, so the $Z$ boson interactions also violate parity. The $A$ field (also known as the $\gamma$, or photon field) interacts with both left- and right-handed fermions with equal strength, and thus does not violate parity. The $W$ and the $Z$ gain a longitudinal degree of freedom and a mass. The theory predicts that the mass of the $W$ and the $Z$ are related by the weak mixing angle: $M_{Z}=M_{W} / \cos \theta_{w}$. The scale of the weak boson masses is set by the vacuum expectation value of $\Phi$ field, and is given by $M_{Z}=\frac{1}{2}(v e v) e / \sin \theta_{w} \cos \theta_{w}$, where $e$ is the charge of an electron. Since the mass of the $Z$ has been very well measured (see table 1.1) we can use it to calculate the vev: $v=M_{Z} \sin \theta_{w} \cos \theta_{w}=246 \mathrm{GeV}$. 


\begin{tabular}{lccc}
\hline \hline Force & Particle & Charge(e) & Mass $\left(\mathrm{GeV} / c^{2}\right)$ \\
\hline Electromagnetic & Photon $(\gamma)$ & 0 & 0 \\
Strong & Gluon $(g)$ & 0 & 0 \\
Weak (charged) & $W$ Boson $\left(W^{ \pm}\right)$ & \pm 1 & 80.3 \\
Weak (neutral) & $Z$ Boson $(Z)$ & 0 & 91.2 \\
Gravitational & Graviton $(G)$ & 0 & 0 \\
\hline
\end{tabular}

Table 1.1: Mass and charge of the gauge bosons in the standard model.

To summarize, the electroweak symmetry is broken because the gauge bosons of the theory interact with a scalar Higgs doublet field that possesses a non-zero vacuum expectation value. This interaction generates a mass and a longitudinal degree of freedom for the $W$ and the $Z$. This can be seen explicitly by transforming the fields into the unitary gauge. The photon does not couple to the scalar field and thus remains a massless boson with only transverse degrees of freedom. The fourth component of the Higgs doublet is a physical scalar particle called the Higgs boson. The Higgs boson has not yet been observed experimentally.

\subsubsection{Fermion Mass}

The fermions in the standard model obtain a mass via a Yukawa coupling to the Higgs doublet. The coupling strength of the fermions to the Higgs doublet determines the mass of the fermions $m_{f}=y_{f} v$, where $y_{f}$ is the coupling constant and $v$ is the vacuum expectation value. Nothing in the theory predicts what this mass will be, so each fermion mass must be measured experimentally. This is one of the unsatisfying features of the standard model, since it introduces several parameters whose value cannot be predicted by the theory.

The massive fermions also acquire a Yukawa coupling to the physical Higgs boson. This coupling is also proportional to the mass of the fermion, so it is quite small for the lighter quarks and leptons. The couplings to the three Higgs degrees of freedom which vanish in the unitary gauge become the coupling of the fermions to the longitudinal mode 
of the gauge bosons. These couplings are also proportional to the mass of the fermion. This implies that the lighter quarks and leptons will couple primarily to the transverse modes, whereas a heavy fermion like the top quark will predominantly couple to the longitudinal mode. This thesis is about the first measurement of the coupling of the top quark to the longitudinal mode of the $W$ boson.

\begin{tabular}{lccc}
\hline \hline Generation & Particle & Charge(e) & Mass $\left(\mathrm{GeV} / c^{2}\right)$ \\
\hline \multirow{3}{*}{ First } & Electron $(e)$ & -1 & $5.1 \times 10^{-4}$ \\
& Electron neutrino $\left(\nu_{e}\right)$ & 0 & $<15 \times 10^{-9}$ \\
& Up quark $(u)$ & $2 / 3$ & $1.5--5 \times 10^{-3}$ \\
& Down quark $(d)-1 / 3$ & $3--9 \times 10^{-3}$ & \\
\hline \multirow{3}{*}{ Second } & Muon $(\mu)$ & -1 & $1.06 \times 10^{-1}$ \\
& Muon neutrino $\left(\nu_{\mu}\right)$ & 0 & $<1.7 \times 10^{-4}$ \\
& Charm quark $(c)$ & $2 / 3$ & $1.1--1.4$ \\
& Strange quark (s) & $-1 / 3$ & $6--17 \times 10^{-2}$ \\
\hline \multirow{3}{*}{ Third } & Tau ( $\tau)$ & -1 & 1.78 \\
& Tau neutrino $\left(\nu_{\tau}\right)$ & 0 & $<1.8 \times 10^{-2}$ \\
& Top quark $(t)$ & $2 / 3$ & $173.8 \pm 5.2$ \\
& Bottom quark $(b)$ & $-1 / 3$ & $4.1--4.4$ \\
\hline
\end{tabular}

Table 1.2: Mass and charge of the fermions in the standard model.

The Yukawa couplings of the fermions to the Higgs sector are independent of the coupling of the fermions to the electroweak gauge sector of the standard model. For quarks, the mass eigenstates and electroweak eigenstates can be related by a matrix called the Cabibo-Kobayashi-Maskawa (CKM) matrix:

$$
\left(\begin{array}{c}
d \\
s \\
b
\end{array}\right)_{\text {weak }}=\left(\begin{array}{ccc}
V_{u d} & V_{u s} & V_{u b} \\
V_{c d} & V_{c s} & V_{c b} \\
V_{l d} & V_{t s} & V_{t b}
\end{array}\right)_{C K M}\left(\begin{array}{c}
d \\
s \\
b
\end{array}\right)_{\text {mass }} \cdot
$$

There are four independent parameters in the CKM matrix, and they are often parametrized as three angles and a complex phase. The complex phase can give rise to charge-parity (CP) violating effects. The elements of the CKM matrix must be determined by experiment. The diagonal elements are all close to 1 , but the off-diagonal elements are non-zero, so quarks 
can change generation in interactions with the $W$ boson. If neutrinos have mass then a similar parameterization will deseribe lepton mass mixing.

\subsection{The Top Quark}

The top quark was discovered in 1995 by the CDF and DO collaborations [2, 3]. The dominant production mode for top quarks at CDF is $q \bar{q} \stackrel{g}{\rightarrow} t \bar{t}$. At Tevatron energies approximately $10 \%$ of the $t \overline{\mathrm{t}}$ pairs are produced via $g g \stackrel{g}{\rightarrow} t \overline{\mathrm{t}}$. Note that in both of these production modes gluons can be radiated from all of the quark and gluon lines involved in the interaction. This gluon radiation is an important source of systematic uncertainty in many top quark measurements, because the gluons can produce hadronic jets that mimic the decay products of the top quark.

In the standard model the top decays almost exclusively to a $b$ quark and $a W$ boson. An interesting feature of the top quark is its mass of $m_{t}=174.3 \pm 5.1 \mathrm{GeV}$, which is considerably more than the mass of the $W$ boson. This allows the top quark to decay to a real $W$ boson, in contrast to all other fermions, which decay to leptonic or hadronic final states via a virtual $W$. Furthermore, as mentioned in the previous section, the massive top quark will decay to the longitudinal mode of the $W$ a large fraction of the time, given by the following equation:

$$
\mathcal{F}_{0}=\frac{\frac{1}{2} m_{t}^{2} / M_{W}^{2}}{1+\frac{1}{2} m_{t}^{2} / M_{W}^{2}},
$$

where $\mathcal{F}_{0}$ is the fraction of longitudinal $W$ bosons that are produced in top decay.

Note that the chiral nature of the coupling of the $W$ requires the $b(\bar{b})$ to be in the right-handed (left-handed) chiral state. The left (right) chiral state is a superposition of the $b$ quark positive and negative helicity states, but since the mass of the $b$ is very small compared to the mass of the $W$ and $t$ the positive (negative) helicity part of the left (right) chiral state will be very small. The $b(\bar{b})$ will thus have negative (positive) helicity, and, in the rest frame of the top quark, this will in restrict the helicity of the $W^{+}\left(W^{-}\right)$to negative (positive) or longitudinal values, because the helicities of the $W$ and the $b$ must sum to $\frac{1}{2}$, 
the spin of the top quark. These considerations in concert with equation 1.3 completely specify the helicity distribution of the $W$ for any given top mass. Any deviation from this predicted helicity distribution would be a violation of the standard model and would thus be very interesting.

\subsubsection{Identifying Top Quark Events}

The top quark and the $W$ boson both decay before they can be observed by the instrumentation of the CDF detector. Thus, we must identify $t \bar{t}$ events by the decay products of the top and the $W$. Both the top and anti-top quarks decay to a bottom (or anti-bottom) quark and a $W$ boson. The $W$ then decays almost instantly into one of the weak isospin fermion doublets which is allowed by the energy of the $W$. The signal for $t \bar{t}$ production depends upon the type of fermions into which the two $W$ bosons decay. There are nine possible decay channels for the $W^{+}, 5$ one for each lepton pair $\left(\overline{e_{R}} \nu_{e L}, \overline{\mu_{R}} \nu_{\mu L}\right.$, and $\left.\overline{\tau_{R}} \nu_{\tau L}\right)$ and three for each quark pair $\left(u_{L} \overline{d_{R}}, c_{L} \overline{s_{R}}\right)$. The quarks are counted three times because they have three color degrees of freedom. The probability of each of the $W$ decay channels is roughly the same, because the mass of all of the final state fermions is very small compared to the mass of the $W$. In the following paragraphs we will discuss each of the top decay channels. Note that events where one or both of the $W$ 's decay leptonically are of particular interest to us, for reasons that we will outline in section 1.4.

If both $W$ bosons decay into quark/anti-quark pairs then the signature for the $t \bar{t}$ event will be six hadronic jets, two of which come from bottom quarks. These are called hadronic top events. These are the most common type of $t \bar{t}$ event, because each $W$ will decay to a hadronic final state $2 / 3$ of the time, and both will thus decay hadronically 4/9 of the time. Unfortunately, hadronic top events are difficult to distinguish from hard QCD scattering events, which are produced copiously at CDF. Furthermore, these events are unsuitable for an analysis of the helicity of the $W$ boson because neither $W$ decay's leptonically.

\footnotetext{
${ }^{3}$ The decay products of the $W^{-}$are the charge and parity conjugates to the decay products of the $W^{+}$.
} 
If one $W$ boson decays hadronically and the other decays to $e \nu_{c}$ or $\mu \nu_{\mu}$ then the signature for the event will be an energetic charged lepton and four hadronic jets (two from bottom quarks) recoiling against large missing transverse energy from the neutrino, which passes through the detector apparatus unobserved. These are called lepton $+j e t$ events. There are a number of other processes which can produce this same signature, but if one or both of the hadronic jets from the $b$ quarks can be identified then the $t \bar{t}$ signal dominates the backgrounds. One nice feature of this decay channel is that it is possible to completely reconstruct the top and anti-top from its daughter particles, up to some combinatoric uncertainties.

If both $W$ bosons decay to $e \nu_{e}$ or $\mu \nu_{\mu}$ then the signature for the event will be two energetic charged leptons and two hadronic jets (both from b quarks) recoiling against large missing transverse energy from the two neutrinos. These are called dilepton events. This signature is a very clean one, because very few alternative processes can mimic it at CDF. Unfortunately, the branching ratio to this final state is only $4 / 81$. Nevertheless, as we shall see, this decay channel is very useful in the analysis of the $W$ boson's helicity, because it is clean and both $W$ 's decay leptonically.

Events where one or both of the $W$ bosons decay to $\tau \nu_{\tau}$ are a special case because the $\tau$ lepton decays quickly and is thus difficult to identify. The hadronic decay channels of the tau, $\tau \rightarrow \bar{u} d \nu_{\tau}, \bar{c} s \nu \tau$, have some distinctive features and some attempts have been made to use these features to identify $t \bar{t}$ events which contain $\tau$ lepton decays. We do not make use of these tau samples in this analysis. However, when the $\tau$ decays leptonically, there is a possibility that the event will pass the selection requirements for the lepton + jet or dilepton data samples.

\subsection{Strategy for Measuring W Helicity}

The chiral nature of the weak interaction is what makes a measurement of the helicity of the $W$ boson possible. The $W$ will decay to a left-handed fermion and a right-handed anti-fermion, and since the $W$ is far more massive than its daughter particles, the right 
and left chiral states of the fermions will correspond almost exactly to their positive and negative helicity states. At tree level, the spins of the $W$ daughters must sum to 1 because angular momentum is conserved in the decay, and the daughters will tend to decay such that the frojection of their spin along the $W$ direction of motion follows the projection of the $W$ 's spin along its direction of motion. Thus, the decay of the anti-fermion, which has positive helicity, will follow the spin projection of the $W$, and the decay of the anti-fermion will be opposite the spin projection of the $W$.

Explicitly, the decay distribution for the fermion in $W$ decay is [5]:

$$
\begin{aligned}
\left|M\left(W_{-}\right)\right|^{2} & =\frac{1}{4}\left(1-\cos \theta_{d}^{*}\right)^{2} \\
\left|M\left(W_{+}\right)\right|^{2} & =\frac{1}{4}\left(1+\cos \theta_{d}^{*}\right)^{2} \\
\left|M\left(W_{0}\right)\right|^{2} & =\frac{1}{2}\left(1-\cos ^{2} \theta_{d}^{*}\right) .
\end{aligned}
$$

In these equations the subscript on the $W$ denotes its helicity, and $\theta_{d}^{*}$ is the angle between the momentum 3-vector of the $W$ in the top rest frame and the momentum 3-vector of the fermion in the $W$ rest frame. The angular distribution of the anti-fermion is given by the replacement $\cos \theta_{d}^{*} \rightarrow-\cos \theta_{d}^{*}$. Note that these equations would be valid for any other definition of $\theta_{d}^{*}$, but we must use the top rest frame in our definition because that is the frame in which we wish to measure the helicity of the $W$.

We need to make a few important observations about these equations:

1. We must know which daughter is the fermion and which is the anti-fermion in order to make full use of these equations. We possess no reliable means for identifying the charge of a quark, so we must restrict ourselves to $t \bar{t}$ events where at least one $W$ decays leptonically, because the charge of the lepton can be measured.

2. We explained in Section 1.3 that the $W^{+}$can have a negative helicity, but not a positive helicity, and that this situation is reversed for the $W^{-}$. Thus, according to equations 1.4 through 1.6 the $\cos \theta_{d}^{*}$ distribution of the $W^{-}$fermion daughter will be the mirror image of the $W^{+}$fermion daughter. However, if we follow the direction of 
the charged lepton instead of the fermion, then the distributions for the $W^{+}$and $W^{-}$ will be the same, because the charged lepton daughter of the $W^{+}$is an anti-fermion whereas the charged lepton daughter of the $W^{-}$is a fermion.

We can now formulate a strategy for measuring the helicity of $W$ bosons in top quark decays. First, we must build a sample of $t \bar{t}$ events which contain leptonic $W$ boson decays. Second, we must reconstruct the angle between the charged lepton's 3-momentum in the $W$ rest frame and the $W$ boson's 3-momentum in the top rest frame. Third, we compare the resulting distribution of $\cos \theta_{d}^{*}$ to equations 1.4 through 1.6 to determine which $W$ helicities are present in the data.

This strategy has drawbacks. To measure $\theta_{d}^{*}$ we must completely reconstruct the $t \bar{t}$ event. This is impossible for dilepton events, because we do not have enough kinematic information to reconstruct the momentum vectors for the two neutrinos. It is possible to fully reconstruct lepton + jet events, but even here there are problems. The event must produce at least four hadronic jets, and we must endeavor to correctly match each of these jets to the quark which produced them. In some cases this is impossible, because one or more of the jets in the event may have originated from a gluon radiated off of one of the quark or gluon lines in the $t \bar{t}$ production diagram. Fortunately, we can afford to mismatch some of the jets as long as we manage to correctly match the jet from the $b$ quark that is recoiling against the leptonically decaying $W$ boson. In $t \bar{t}$ events where we have identified at least one jet as having come from a $b$ quark our chance of correctly matching the lepton to the $b$ jet is approximately $50 \%$.

Another concern is that this strategy relies on our ability to reconstruct the $W$ rest frame, which means that we must determine the energy and momentum of the neutrino. However, we can observe the neutrino only through the recoil of all other objects in the $t \overrightarrow{\mathrm{t}}$ decay from the neutrino. Thus, the precision of our measurement of the neutrino momentum depends upon the precision of our measurement of every other object in the event, and this means that the neutrino momentum is poorly measured. 


\subsubsection{Using lepton $P_{T}$}

Because of these considerations we have devised an alternative strategy. Note that equations 1.4 through 1.6 imply that the charged lepton will decay in the opposite direction of the $W$ momentum (in the top rest frame) if the $W$ is not longitudinal, and it will decay perpendicular to the $W$ momentum if the $W$ is longitudinal. It turns out that this affects the momentum distribution of the charged lepton in the rest frame of the CDF detector (henceforth referred to as the lab frame). This is very convenient, because the lepton momentum spectrum is the most precisely measured quantity in a $t \bar{t}$ event. We show the predicted lab frame lepton transverse momentum $\left(P_{T}\right)^{6}$ distributions for the three $W$ helicities in Figure 1.1. The three distributions are distinctive, so we should be able to exploit them to determine the $W$ helicity.

Our strategy then is first to compose a selection of $t \bar{t}$ events where at least one of the $W$ bosons decays leptonically. Second, we plot the distribution of the transverse momentum $\left(\boldsymbol{P}_{T}\right)$ of the charged lepton. Finally, we compare the resulting $\boldsymbol{P}_{T}$ distribution to Figure 1.1 to determine the amount of each $W$ helicity in our data. The remainder of this thesis describes in detail the implementation of this plan.

In chapter 2 we describe the CDF detector plant. Event selection is described in chapter 3 and we describe the Monte Carlo samples which are used in this analysis in chapter 4. The methods we use to measure the helicity of the $W$ boson are outlined in chapter 5 and the systematic uncertainties in the measurement are discussed in chapter 6.

\footnotetext{
'The transverse momentum of a particle is the projection of its momentum in the plane perpindicular to the beam line. We use this quantity instead of the momentum because the momentum of the initial state partons along the beamline is unknown in pp collisions.
} 


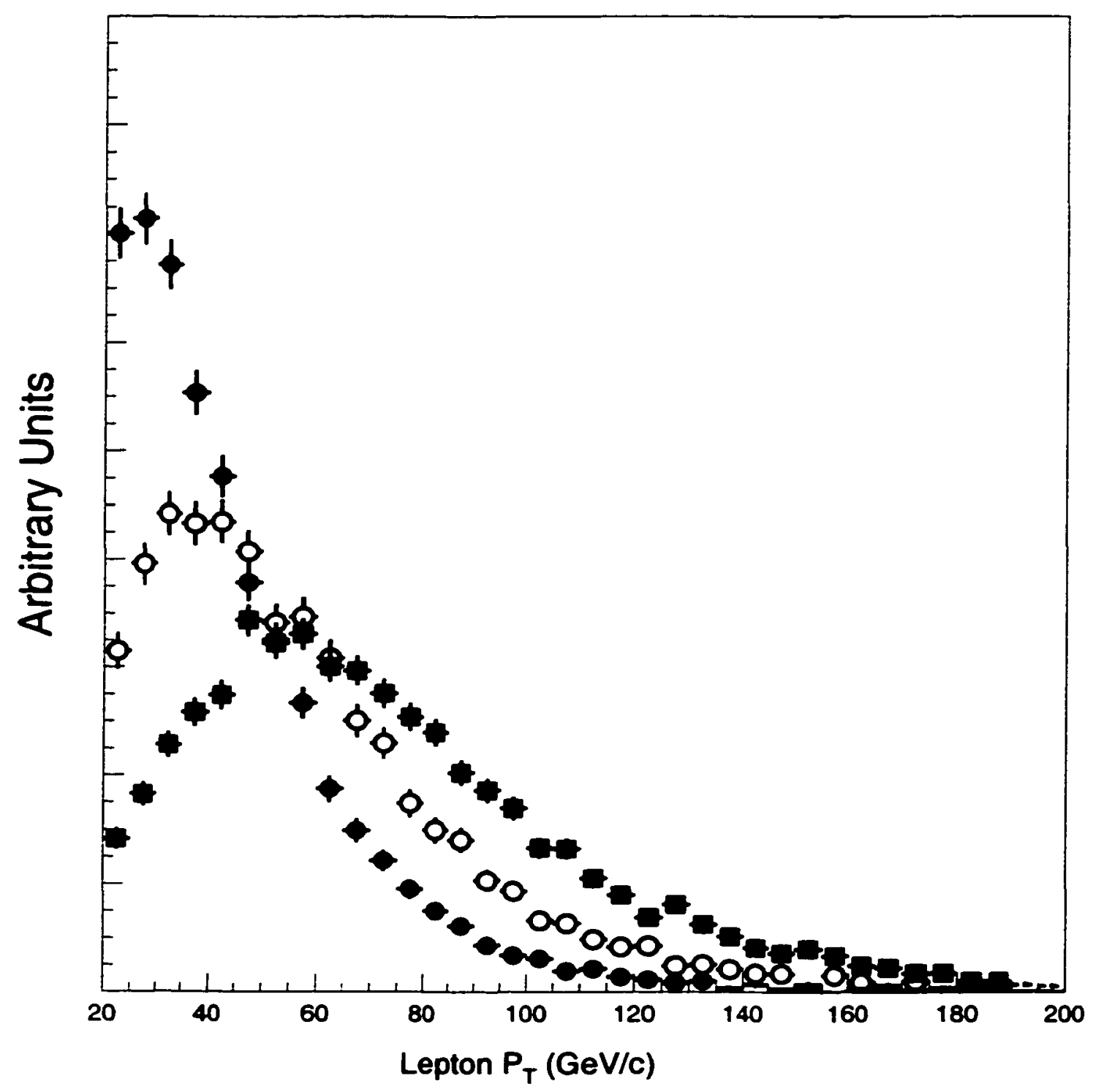

Figure 1.1: Lepton $P_{T}$ distributions for the three $W$ helicities. The solid.circles are from negative helicity $W^{+}$and positive helicity $W^{-}$, the open circles are from longitudinal $W^{+}$ and $W^{-}$, and the closed squares are from positive helicity $W^{+}$and negative helicity $W^{-}$. All three distributions are normalized to the same area. 


\section{Chapter 2}

\section{The Fermilab Tevatron and the CDF Detector}

The Tevatron at Fermi National Accelerator Laboratory (FNAL) is a superconducting particle accelerator which produces high energy protons $(p)$ and antiprotons $(p)$. These high energy particles can be utilized in two ways. The protons can be supplied to a number of "fixed target" detector plants which study the debris from a collision between the protons and a stationary target. Alternatively, the protons and antiprotons can be collided at a center of mass energy of up to $\sqrt{s}=1.8 \mathrm{TeV}$. These $\mathrm{p} \overline{\mathrm{p}}$ collisions are studied by two detector facilities, the Collider Detector at Fermilab (CDF) and the D0 detector plant. The data used in the measurement of the helicity of $\boldsymbol{W}$ bosons in top quark decays were collected by CDF during two collider runs: run $1 A$ (1992-1993) and run $1 B$ (1994-1996).

In this chapter we describe the collider mode of the Tevation and the CDF detector apparatus.

\subsection{The Tevatron}

The Tevatron accelerator complex is depicted schematically in Figure 2.1 ${ }^{1}$. The protons for a collider run come from a hydrogen gas $\left(H_{2}\right)$ which is ionized to form $H^{-}$ and accelerated to $750 \mathrm{keV}$ by a Cockroft-Walton electrostatic accelerator in the PreAcc. The $H^{-}$ions are then injected into the Linac, which uses radio-frequency (RF) chambers

\footnotetext{
'Accelerator elements that are pictured in this figure are italicised when they first appear in the text
} 
to further accelerate them to an energy of $400 \mathrm{MeV}$. The ions are passed through a thin carbon foil to scrape the electrons off and thus form protons, which are then injected into the Booster accelerator. In the Booster the protons are accelerated to an energy of $8 \mathrm{GeV}$ and are gathered into bunches. The bunches are injected into the Main Ring, a synchrotron of $1 \mathrm{~km}$ radius. In the Main Ring the bunches are accelerated to an energy of $150 \mathrm{GeV}$ and are then coalesced into a single bunch and finally injected into the Tevatron, its final destination. The Tevatron is the same size as the Main Ring and is located directly below it. The Tevatron uses superconducting magnets cooled by liquid helium to at tain the large magnetic fields that it needs to contain $900 \mathrm{GeV}$ beams of protons and antiprotons.

The formation of antiproton bunches is considerably more complicated. To create antiprotons, protons are accelerated to $120 \mathrm{GeV}$ in the Main Ring and are then collided with a tungsten target in the PBar Target area. This collision produces a menagerie of exotic particles which includes some antiprotons. These antiprotons are separated from the other particles, focused by a lithium lens, and then injected into the Debuncher. In the Debuncher the antiprotons are stochastically cooled before they are transferred to the Accumulator, where they are stored at an energy of $8 \mathrm{GeV}$. The antiprotons are "stacked" into the accumulator until roughly $10^{12}$ are accumulated. This process takes about a day. The antiprotons are finally injected into the Main Ring in six bunches of roughly $3 \times 10^{10}$ particles. These bunches are accelerated to $150 \mathrm{GeV}$ in the Main Ring, and are then inserted into the Tevatron, where they attain an energy of $900 \mathrm{GeV}$. The antiprotons orbit the Tevatron in the opposite direction from the protons. Their paths cross at the $B O$ and $D O$ interaction regions, where the collisions are recorded by the CDF and DO detector plants, respectively. The typical instantaneous luminosity delivered to the $\mathrm{BO}$ interaction point was $0.5 \times 10^{31} \mathrm{~cm}^{-2} \mathrm{~s}^{-1}$ during Run $1 \mathrm{~A}$ and $1.6 \times 10^{31} \mathrm{~cm}^{-2} \mathrm{~s}^{-1}$ during Run $1 B$.

\subsection{The Collider Detector at Fermilab}

The Collider Detector at Fermilab (CDF) is a general purpose machine that was designed to study the debris produced in $\mathbf{p} \overline{\mathbf{p}}$ collisions in the Tevatron. The design and 


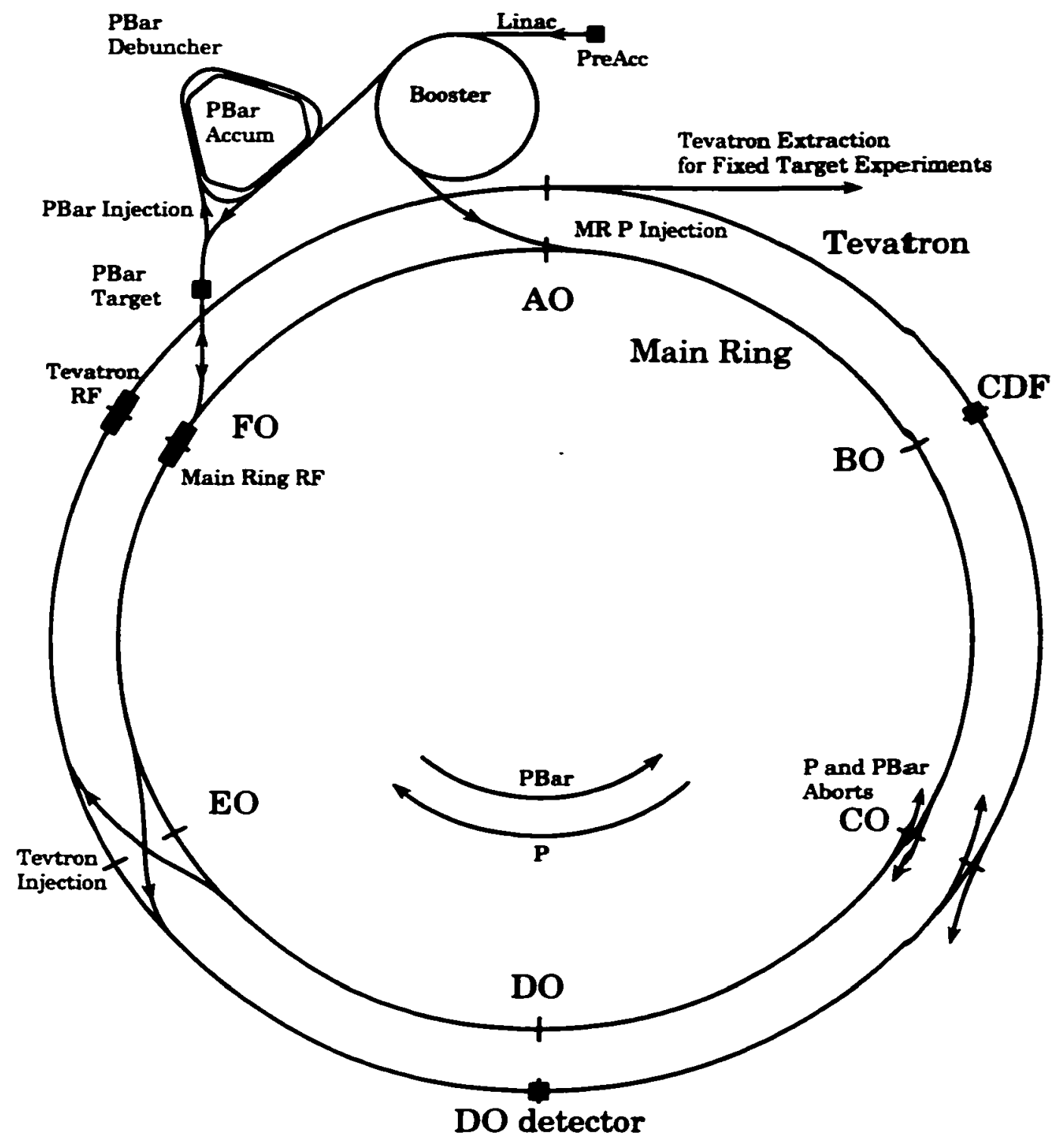

Figure 2.1: A schematic diagram of the Tevatron collider complex. 
function of the CDF detector plant is described in detail in [6]. We describe in this section the detector components that were most useful in the analysis of the $W$ helicity. We depict in Figure 2.2 a cross-sectional schematic of the CDF detector. The apparatus is cylindrically symmetric about the beam line and forward-backward symmetric across the plane through the interaction point and transverse to the beam-line, so only a quarter of the detector is pictured. The CDF coordinate system uses $(\eta, \phi, z)$, where $z$ an axis along the proton beam line, $\phi$ is the azimuthal angle, and $\eta$ is a variable called "pseudorapidity" and is defined by $\eta=-\log [\tan (\theta / 2)]$, where $\theta$ is the polar angle. The origin of this coordinate system is at the nominal interaction point.

The detector consists of a number of different subsystems which work in concert to identify and then measure the properties of the particles which are produced in p$\overline{\mathbf{p}}$ collisions. These subsystems can generally be divided into four categories: tracking, calorimetry, muon chambers, and triggering.

The tracking systems are located inside of a $1.5 \mathrm{~m}$ radius superconducting solenoid which produces a 1.4T magnetic field that aids in the identification of the charge and the momentum of the particles which leave tracks in the tracking chambers. The tracking system closest to the beamline is the Silicon Vertex Detector (SVX), which provides precise tracking information in the plane transverse to the beamline and is used to identify secondary vertices from $B$ hadron decays. The SVX is mounted inside of the Vertex Time Projection Chamber (VTX), which provides information on the $z$-position of the primary interaction vertex. Surrounding the VTX is the Central Tracking Chamber (CTC). The CTC is a cylindrical drift chamber which provides three dimensional tracking of charged particles with a momentum resolution of $\delta P_{T} / P_{T}^{2} \approx 0.002 \mathrm{GeV} / \mathrm{c}^{-1}$.

The calorimetry systems are located outside of the solenoid, and are used to measure the electromagnetic and hadronic energy of photons, electrons, and hadronic jets. The calorimeters are divided into electromagnetic (CEM, PEM, FEM) and hadronic (CHA, WHA, PHA, FHA) components. Finally, located outside the calorimeters are three drift chambers (CMU, CMP, CMX) which are used to identify muons. 


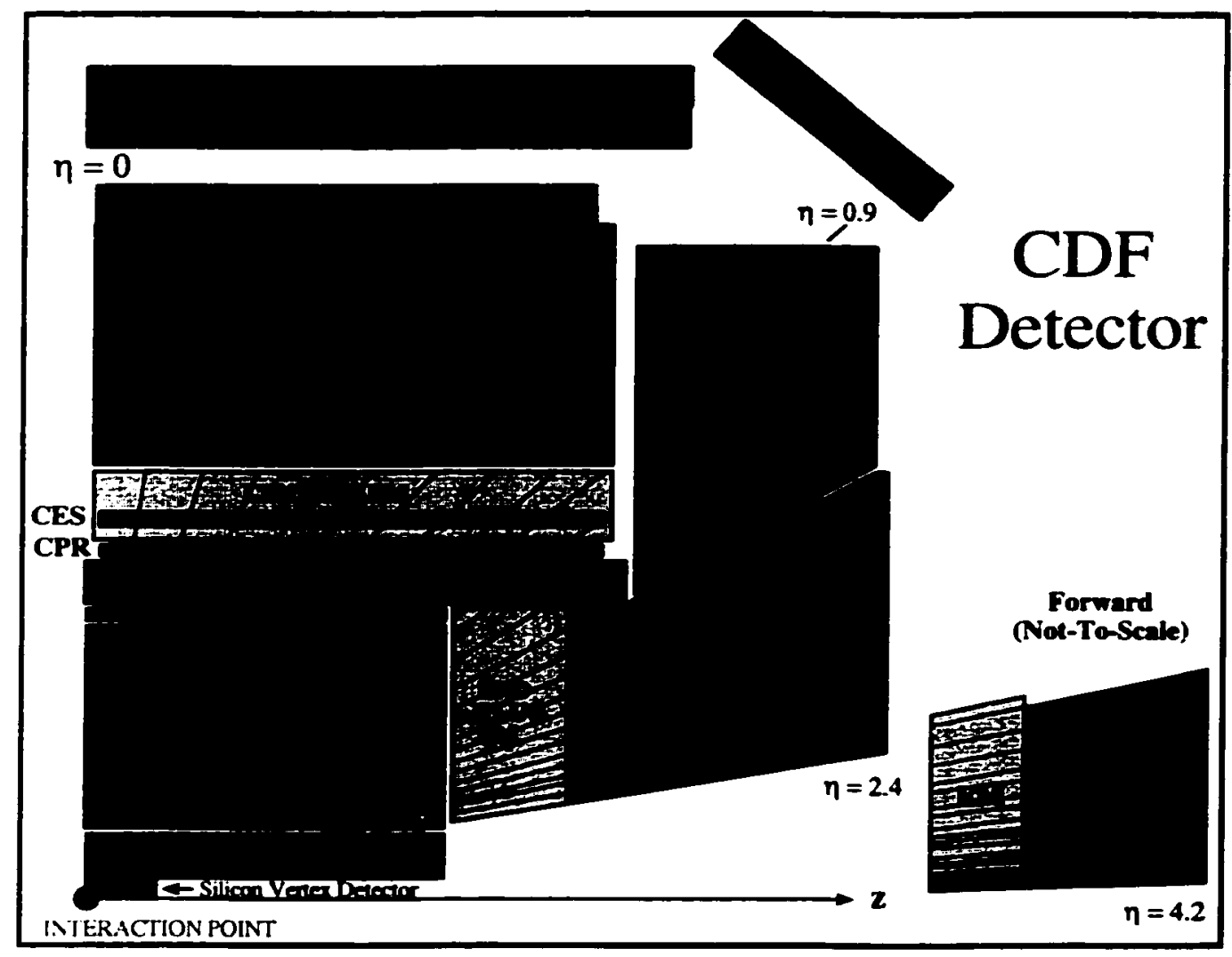

Figure 2.2: A schematic diagram of the CDF detector plant. Note that CDF has a cylindrical symmetry and that this picture shows only a quarter of the detector. For the entire detector rotate this picture about the beamline and add its mirror image across the left side of the picture. 
Information from these systems is supplied to a three level trigger which is used to select in real time the events which are to be recorded for further study.

\subsubsection{The Tracking Systems}

The tracking systems at CDF are enclosed within a $3 \mathrm{~m}$ diameter superconducting solenoid [7] which provides a 1.4T magnetic field along the beamline. The solenoid is $4.8 \mathrm{~m}$ in length. The magnetic field causes charged particles to move in a helical trajectory. The charge and transverse momentum of a particle can be derived by measuring the degree of curvature in its track.

The SVX $[8,9]$ is the tracking system located closest to the Tevatron beampipe (radius $1.9 \mathrm{~cm}$ ). The SVX consists of two identical barrels which are located to either side of the nominal interaction point $(z=0)$. Each barrel is composed of 4 layers which are segmented azimuthally into 12 wedges. Each of the 4 layers within a wedge are constructed of silicon microstrip detectors that are bonded together into groups of three called ladders. The innermost layer had a radius of $3.0 \mathrm{~cm}$ in run IA and a radius of $2.86 \mathrm{~cm}$ in run IB. The outermost layer had a radius of $7.87 \mathrm{~cm}$. The total active length of the $\mathrm{SVX}$ is $51 \mathrm{~cm}$, which corresponds to a pseudorapidity coverage of $|\eta|<1.9$. The SVX can measure track impact parameters relative to the beam position with a precision of $17 \mu \mathrm{m}$. This makes the SVX a crucial part of CDF's ability to identify $B$ mesons, which have a mean decay length of $c \tau \approx 480 \mu \mathrm{m}$.

The next tracking chamber, the VTX [10], is used to measure the location of the primary interaction vertex along the $z$-axis. The VTX has an inner radius of $8 \mathrm{~cm}$, an outer radius of $22 \mathrm{~cm}$, and has a pseudorapidity coverage of $|\eta|<3.25$. The VTX can find the event vertex with a precision of about $1 \mathrm{~mm}$. The distribution of primary vertices along the z-axis has been found to describe a Gaussian of width $30 \mathrm{~cm}$.

Surrounding both the SVX and the VTX is the CTC. The CTC [11] is a $3.2 \mathrm{~m}$ long cylindrical drift chamber with an inner radius of $31 \mathrm{~cm}$ and an outer radius 0 $132.5 \mathrm{~cm}$. The CTC has a pseudorapidity coverage of $|\eta|<1.1$. The chamber contains 84 layers of 
sense wires that are organized into 9 superlayers. The superlayers come in two types, axial and stereo. The five axial superlayers consist of 12 layers of sense wires oriented parallel to the beamline. These layers are interleaved with the four stereo superlayers, which are composed of six layers of sense wires which are oriented with a tilt of $\pm 3^{\circ}$ relative to the beamline. The axial layers provide accurate tracking in the $r-\phi$ plane, while the tilt of the stereo layers provides some information on the $z$ position of a particles track. The CTC, in concert with the SVX, can measure the transverse momentum of a track with a resolution of $\delta P_{T} / P_{T}=0.0011 P_{T}$.

\subsubsection{Calorimetry}

Calorimetry systems are located outside of the solenoid at CDF. These systems are divided into three major regions of $|\eta|$ coverage: central, plug, and forward. The calorimeters in each of these regions are organized in a tower geometry. Each tower consists of an electromagnetic calorimeter placed in front of a hadronic calorimeter. These towers are projective, which means that they point back to the nominal interaction region. The hadronic calorimeters have a somewhat larger cross-sectional area than the electromagnetic calorimeters in order to give both calorimeters in the tower the same coverage in $\eta-\phi$ space. In the central region the towers are $15^{\circ}$ wide in $\phi$ and 0.1 units wide in $\eta$, while the towers in the forward and plug regions are $5^{\circ}$ wide in $\phi$ and 0.1 units wide in $\eta$. The coverage and energy resolutions of the various components which make up the CDF calorimetry system are summarized in Table 2.1.

The central electromagnetic calorimeter (CEM) $[12,13,14,15,16]$ towers are composed of alternating layers of lead absorber and scintillator with a total thickness of 18 radiation lengths. The energy resolution of the $\mathrm{CEM}$ is $\sigma(E) / E=13.5 \% / \sqrt{E \sin \theta}$, where $E$ is measured in $\mathrm{GeV}$. Embedded within the CEM at the approximate shower maximum depth of six radiation lengths is a system of proportional chambers with strip and wire readouts, called the CES. The CES provides shower shape and position information in both $z$ and $r-\phi$. Another set of proportional chambers, the CPR, is located between the CEM 
and the solenoid. The CPR samples the early development of an electromagnetic shower within the solenoid.

The CEM is surrounded by the central hadronic calorimeter (CHA) [17] and the wall hadronic calorimeter (WHA). The CHA shares the cylindrical geometry of the CEM and in fact is contained within the same physical wedges that house the CEM. The geometry of the WHA is considerably different from the CHA because its function is to cover the edge of a cylindrically shaped region. Both detectors are composed of alternating layers of iron and plastic scintillator. The pseudorapidity coverage of the CHA is $|\eta|<0.9$ and the coverage of the WHA is $0.9<|\eta|<1.3$.

The calorimetry in the endplug region is handled by the plug electromagnetic calorimeter (PEM) [18] and the plug hadronic calorimeter (PHA) [19]. Both systems share the same projective geometry and segmentation. These systems are adjacent to the CEM and the WHA/CHA and extend the pseudorapidity coverage of the central region of the CDF detector calorimetry out to $|\eta|<2.4$. The active region in both calorimeters is a system of argon-ethane gas proportional chambers. These proportional tube arrays are interleaved with lead in the PEM and iron in the PHA.

The pseudorapidity coverage of the calorimeter systems is extended out to $|\eta|<4.2$ by the forward electromagnetic calorimeter (FEM) [20] and forward hadronic calorimeter (FHA) [21]. These calorimeters are composed of two modules which are physically separated from the CDF detector proper and are positioned $6.5 \mathrm{~m}$ upstream and downstream of the nominal interaction point. The active and inactive elements of these detectors are similar to their counterparts in the plug region.

\subsubsection{Muon Detectors}

There are three muon detector systems which are germane to this analysis, the central muon chambers (CMU), the central muon upgrade (CMP), and the central muon extension (CMX). These chambers provide muon identification and crude momentum information to the CDF trigger, and work in concert with the tracking systems to provide 


\begin{tabular}{cccc}
\hline \hline System & $\eta_{d}$ Range & Energy Resolution & Thickness \\
\hline CEM & $\left|\eta_{d}\right|<1.1$ & $13.7 \% / \sqrt{E_{T}} \oplus 2 \%$ & $18 X_{0}$ \\
PEM & $1.1<\left|\eta_{d}\right|<2.4$ & $22 \% / \sqrt{E_{T}} \oplus 2 \%$ & $18-21 X_{0}$ \\
FEM & $2.2<\left|\eta_{d}\right|<4.2$ & $26 \% / \sqrt{E_{T}} \oplus 2 \%$ & $25 X_{0}$ \\
CHA & $\left|\eta_{d}\right|<0.9$ & $50 \% / \sqrt{E_{T}} \oplus 3 \%$ & $4.5 \lambda_{0}$ \\
WHA & $0.7<\left|\eta_{d}\right|<1.3$ & $75 \% / \sqrt{E_{T}} \oplus 4 \%$ & $4.5 \lambda_{0}$ \\
PHA & $1.3<\left|\eta_{d}\right|<2.4$ & $106 \% / \sqrt{E_{T}} \oplus 6$ & $5.7 \lambda_{0}$ \\
FHA & $2.4<\left|\eta_{d}\right|<4.2$ & $137 \% / \sqrt{E_{T}} \oplus 3$ & $7.7 \lambda_{0}$ \\
\hline \hline
\end{tabular}

Table 2.1: Summary CDF calorimetry properties. The symbol $\oplus$ signifies addition in quadrature. Energy resolutions for the electromagnetic calorimeters are for incident electrons and photons; for the hadronic calorimeters, they are for incident isolated pions. Energy is in $\mathrm{GeV}$. Thicknesses are given in radiation lengths $\left(X_{0}\right)$ for electromagnetic calorimeters, and in interaction lengths $\left(\lambda_{0}\right)$ for hadronic calorimeters.

accurate muon momentum information for use in data analysis. All three systems are four layer drift chambers.

The CMU is located immediately outside of the CHA. The CHA acts as a hadron absorber for the CMU, but there is still a considerable number of hadrons which punch through and leave a track in the CMU. An additional 0.6 meters of steel (3.5 $\left.\lambda_{0}\right)$ surrounds the CMU and is in turn surrounded by the CMP. The steel reduces hadronic punchthrough to the CMP by a factor of 30. For various reasons, the CMU and CMP do not completely cover the central region of the CDF detector. The CMU covers about $84 \%$ of the solid angle, while the CMP covers $63 \%$ of the solid angle. The simultaneous coverage of the two systems is $53 \%$ of the solid angle.

The CMX chambers provide additional muon coverage in the pseudorapidity region from $0.6<|\eta|<1.0$. The drift chambers are placed on four free standing conical arches. Much like the CMU/CMP systems, there are gaps in the coverage of the CMX. The coverage is roughly $71 \%$ of the solid angle in that pseudorapidity region. 


\subsubsection{The CDF Trigger System}

The Tevatron delivers an event rate of approximately $280 \mathrm{kHz}$ to the CDF detector. In contrast, the CDF data aquisition system is capable of reading out the detector and storing all relevant event information at a rate of 6 to $8 \mathrm{~Hz}$. CDF uses a three tier trigger system to select the events which are most likely to be interesting for data readout and storage. The three levels of the trigger system are engaged sequentially, and if an event does not pass the trigger algorithm for one of the levels then it does not get passed on to the next level in the sequence and no further analysis of that event is performed.

The level 1 trigger was designed to arrive at its decision between bunch crossings,

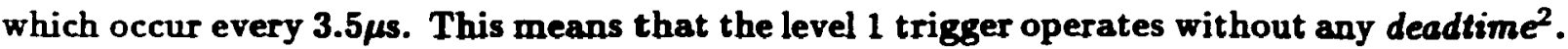
The quick decision is possible because the entire trigger was implemented in dedicated hardware. The relevant level 1 triggers for the top analysis are the muon triggers and the calorimeter triggers. The muon triggers use fast outputs from the CMU, CMP, and CMX, to identify muon candidates, but no effort is made to match muon chamber hits to tracks in the CTC. In fact, no CTC information is available to the level 1 trigger. The calorimeter triggers separately sum the electromagnetic and hadronic energy in trigger towers of $(\Delta \eta=0.2) \times\left(\Delta \phi=15^{\circ}\right)$. The hadronic or electromagnetic energy in a trigger tower must pass a certain threshold to be accepted. The level 1 trigger typically reduces the event rate from $280 \mathrm{kHz}$ down to $1 \mathrm{kHz}$.

The level 2 trigger performs a more sophisticated analysis of events which are accepted by the level 1 trigger. Dedicated hardware forms calorimeter clusters by searching for a trigger tower above a certain seed threshold, and then adds adjacent towers that are above a lower threshold. Another dedicated hardware system, the central fast tracker (CFT) uses information from the five axial superlayers of the CTC to measure the transverse momentum of tracks with a resolution of $\delta P_{T} / P_{T} \approx 0.035 \mathrm{GeV}^{-1} \cdot P_{T}$ and an efficiency of $(93.5 \pm 0.3) \%$ for events with $P_{T}$ above $10 \mathrm{GeV} / c$. These CFT tracks can be matched to ealorimeter clusters to form electron candidates or to CMU, CMP, CMU/CMP, or CMX

\footnotetext{
${ }^{2}$ Dead time is the fraction of beam crousings during which the detector is not operating
} 
hits to form muon candidates. The final trigger decision is made with software which runs on a DEC Alpha 21064 processor. Depending on the complexity of the event, the level 2 trigger can take from 10's to 100's of microseconds. During this time the level 1 trigger cannot accept new events, so the level 2 trigger incurs a downtime of about $4 \%$. The level 2 trigger reduces the event rate of $1 \mathrm{kHz}_{2}$ from the level 1 trigger down to 20 to $35 \mathrm{~Hz}$.

The level 3 trigger is software based and runs on a farm of eight Silicon Graphics processors. It bases its decision on a complete readout of the detector instead of the fast readouts that were available to the level 1 and level 2 triggers. The level 3 trigger algorithms perform a simplified version of the offline event reconstruction that is used in data analysis. The level 3 trigger and data aquisition systems incur a dead time of about $10 \%$. The accept rate is about $5-8 \mathrm{~Hz}$, and all accepted events are written to $8 \mathrm{~mm}$ magnetic tapes for future study. 


\section{Chapter 3}

\section{Event Selection}

This measurement is a shape analysis of the $P_{T}$ distribution of leptons. Thus we require a sample of events where at least one top decays to a lepton via the decay chain $t \rightarrow b W \rightarrow b l \nu$. Both the detector signature and the backgrounds for $t \bar{t}$ events depend very strongly upon whether both top quarks decay to leptons, or just one of them does. Events where only one top decays to a semi-leptonic final state and the other decays hadronically are called "lepton + jet" events, because the signature for these events is a high $P_{T}$ lepton that is recoiling off of three or more jets and large $\mathbb{W}_{T}$. Events where both top quarks decay to a semi-leptonic final state are called "dilepton" events. The signature for these events is two high $P_{T}$ leptons, large $\boldsymbol{F}_{T}$, and two or more hadronic jets. We discuss both of these samples in detail below.

Note that we do not include events in which the $W$ decays to a $\tau$ lepton in our lepton + jet or dilepton samples. This is because the $\tau$ is a short lived particle that decays to a variety of final states that mimic other processes, thus making it a difficult particle to identify. Throughout the rest of this thesis it should be understood that we use the word lepton to mean only electrons and muons, unless we specifically refer to the $\tau$ lepton as well.

This chapter is organized as follows. First we describe the selection of a large sample of high- $P_{T}$ inclusive leptons. This sample is the starting point for the creation of both the dilepton and lepton+jet samples. In the following chapters we discuss separateiy 
the selection requirements for the lepton+jet samples and the dilepton sample, and the backgrounds to both. The efficiencies and motivations for these selection are discussed briefly in this chapter, and are well documented in $[22,23,24,25]$.

\subsection{Inclusive High $P_{T}$ Electrons}

We consider only electrons which deposit energy in the central calorimeter region $(|\eta|<1.0)$. The electron is expected to deposit most of its energy in a single electromagnetic calorimeter tower. It should also leave a track in the CTC which points to the associated CEM cluster. Finally, it should have a well defined shower profile in the CES.

\subsubsection{Electron Triggers}

Electron events are collected online using the three tiered CDF trigger system that is outlined in Section 2.2.4. The exact thresholds used differed between the two data taking runs (run Ia and run Ib).

The level 1 electron trigger required one CEM or CHA tower above $6 \mathrm{GeV}$ or 8 $\mathrm{GeV}$, respectively, in run Ia. In run $\mathrm{Ib}$ the thresholds were raised to $8 \mathrm{GeV}$ and $12 \mathrm{GeV}$, respectively, in order to cope with the increased instantaneous luminosity delivered during this run.

During run Ia, the level 2 electron trigger required a CFT track with $P_{T}>9.2 \mathrm{GeV} / \mathrm{c}$ to be associated with a cluster of CEM trigger towers with $E_{T}>9 \mathrm{GeV}$. During run Ib these thresholds were lowered to $7.5 \mathrm{GeV} / c$ and $8 \mathrm{GeV}$, respectively.

The level 3 trigger uses three dimensional track reconstruction, and requires a track with $P_{T}>13 \mathrm{GeV} / c$ for run $\mathrm{Ia}$ and $P_{T}>10 \mathrm{GeV} / c$ for run $\mathrm{Ib}$ to point at a CEM cluster with $E_{T}>20 \mathrm{GeV}$ for run Ia and $E_{T}>18 \mathrm{GeV}$ for run Ib.

\subsubsection{Offline Electron Selection}

The following cuts are applied offline to select the high $P_{T}$ lepton sample: 
- $E_{T}>20 \mathrm{GeV}$

- $E / P<1.8$

- The ratio of the energy deposited by the electron in the CEM to the momentum of the electron is expected to be near unity. In Run Ia this ratio was required to be less than 1.5. This cut was loosened to 1.8 for Run Ib.

- $\frac{E_{\mathrm{HAO}}}{E_{\mathrm{EM}}}<0.05$

- Electromagnetic showers generally occur within the first few radiation lengths of the CEM and consequently deposit very little energy in the hadronic calorimeters. Thus we insist that the ratio of the energy that is deposited in the CHA or WHA to the energy that is deposited in the CEM be less than 0.05 .

- $L_{s h r}<0.2$

- The variable $L_{\text {shr }}$ is the lateral shower profile for electrons. It compares the energy in the electromagnetic cluster's seed tower to that of adjacent CEM towers, and we require that this profile resemble that seen in test beam electrons. When $L_{\text {ghr }}=0$ the match with test beam electrons is exact. $L_{\text {shr }}$ is defined as: $L_{\mathrm{shs}}=0.14 \sum_{i} \frac{E_{i}^{o b s}-E_{i}^{\exp }}{\sqrt{(0.14 \sqrt{E})^{2}+\sigma_{E_{i}^{2}}^{\exp }}}$. Where the sum is over towers adjacent to the seed tower, $E_{i}^{a b s}$ is the energy deposited in the CEM tower by the electron candidate, $E_{i}^{e x}$ is the energy expected from test beam electrons, $0.14 \sqrt{E}$ is the CEM energy resolution, and $\sigma_{E_{i}^{e x p}}^{2}$ is the uncertainty in $E_{i}^{\text {exp }}$.

- $|\Delta x|<1.5 \mathrm{~cm}$.

- We expect all electrons to have an EM shower in the CEM accompanied by a matching charged track in the CTC. $\Delta x$ is the distance in the $r-\phi$ plane between the CES position of the electron candidate and the position of the extrapolation of its CTC track to the CES. 
- $|\Delta z|<3.0 \mathrm{~cm}$

- $\Delta z$ is the distance in the $r-z$ plane between the CES chamber position and the CTC track position.

- $\chi_{\text {strip }}^{2}<10$.

- This is the $x^{2}$ from a comparison of the shower profile in the CES to that which is expected from test electrons.

- $\left|Z_{v}-Z_{0}^{e}\right|<5 \mathrm{~cm} ;\left|Z_{v}\right|<60 \mathrm{~cm}$.

- $Z_{v}$ is the displacement along the beam line of the primary vertex in the event from the center of the detector and $Z_{0}^{e}$ is the displacement of the electron along the beamline.

- Fiducial cuts.

- The electron must deposit most of its energy in the CEM. We require that the shower position, as measured by the CES, be well away from CEM wedge boundaries and other well known inactive regions.

- Conversion removal.

- Photons that convert to an $e^{+} e^{-}$pair in the material that precedes the CTC are removed if there are few hits in the VTX, or if the charged track can be matched to another track and summed to a small invariant mass that originates inside of the CTC.

\subsection{Inclusive High $P_{T}$ Muons}

Muon candidates are identified in the central rapidity region of the detector $(|\eta|<$ 1.0). We use information from the CTC and the muon chambers (CMU, CMP, and CMX) 
for both the trigger decisions and the offline selection. Furthermore, during offline reconstruction of muon events we consider the energy that is deposited in the calorimeter by the muon candidate.

In general, muon candidates are expected to have a track in the CTC which points to hits in one or more of the muon chambers. The energy deposition in the calorimeter tower(s) which the muon passes through is expected to be consistent with a minimum ionizing particle.

\subsubsection{Muon Triggers}

The level 1 muon trigger requires muon chamber hits with a minimum $P_{T}$ of 6 $\mathrm{GeV} / c$ in the $\mathrm{CMU}$ and $10 \mathrm{GeV} / c$ in the $\mathrm{CMX}$. Muon segments in the CMU require CMP confirmation for those sections of the CMU with CMP coverage.

The level 2 muon trigger requires a CFT track with $P_{T}>9.2 \mathrm{GeV} / c$ for run la and $P_{T}>12 \mathrm{GeV} / c$ for run $\mathrm{Ib}$ to be matched in the $r-\phi$ plane with a muon segment. At level 3 a more precise match was required.

\subsection{Offline Muon Selection}

The following selection cuts are applied to create the high $\boldsymbol{P}_{T}$ muon sample:

- $P_{T}>20 \mathrm{GeV} / c$.

- EM Energy $<2.0 \mathrm{GeV}$.

- The energy deposited in the CEM tower that the muon candidate passes through must be less than $2.0 \mathrm{GeV}$.

- HAD Energy $<6.0 \mathrm{GeV}$. 
- The energy deposited in the CHA tower that is associated with the muon candidate must be less than $6.0 \mathrm{GeV}$. This threshold is higher than that for the CEM because the CHA contains more material for the muon to interact with.

- $\mathrm{EM}$ energy + HAD energy $>0.1 \mathrm{GeV}$.

- $D_{0}<3 \mathrm{~mm}$

- The closest approach of the reconstructed muon track to the beamline $\left(D_{0}\right)$ must be less than $3 \mathrm{~mm}$.

- $\left|Z_{v}-Z_{0}^{\mu}\right|<5 \mathrm{~cm}$

- The $Z$ position of the muon track $\left(Z_{0}^{\mu}\right)$ must be within $5 \mathrm{~cm}$ of the $Z$ position of the event vertex $\left(Z_{v}\right)$.

- $\left|Z_{v}\right|<60 \mathrm{~cm}$

- CTC - Muon chamber matching requirements: the extrapolation of the CTC track to the muon chambers must fall within a certain distance $\Delta x$ of the associated hits in the muon chambers.

- CMU: $|\Delta x|<2.0 \mathrm{~cm}$ or CMP: $|\Delta x|<5.0 \mathrm{~cm}$.

$-\mathrm{CMX}:|\Delta \boldsymbol{x}|<5.0 \mathrm{~cm}$.

\subsection{Lepton + Jet Event Selection}

The lepton + jet sample is defined by the following selection criteria:

- One high $P_{T}$ lepton (as defined above).

- $Z$ removal (defined below).

- Lepton calorimeter isolation $\left(I_{c a l}<0.1\right)$. 
- We require the calorimeter isolation $\left(I_{c a l}\right)$ to be less than 0.1 . The calorimeter isolation is defined as the ratio of energy deposited in the calorimeter within a cone of radius $\Delta R=\sqrt{\Delta \phi^{2}+\Delta \eta^{2}}=0.4$ of the lepton (but excluding the energy of the lepton itself) to the energy of the lepton.

- $E_{T}>20 \mathrm{GeV}$.

- Three or more jets with $E_{T}>15 \mathrm{GeV}$ and $|\eta|<2.0$.

When these selection requirements are applied to CDF run I data we are left with 324 events, with an estimated background of $272.4 \pm 14.0$ events. To enhance the signal to background ratio in this sample, we construct three separate sub-samples with additional selection criteria. These subsamples are discussed in Sections 3.4.2 through 3.4.4.

\subsubsection{Z removal}

$Z$ boson events can mimic lepton + jet production if the $Z$ decays to $l^{+} l^{-}$and one of the leptons passes the high $\boldsymbol{P}_{T}$ lepton selection requirements. To eliminate $\boldsymbol{Z}$ bosons from our lepton + jet sample we look for a secondary lepton in the event.

- If the primary lepton is an electron then the secondary lepton must be an electron in the forward, central, or plug calorimeter that meets the following criteria:

$-E_{T}>10 \mathrm{GeV} / c^{2}$

$-E / P>2.0$ for central electrons,

$-E_{\text {had }} / E_{\text {em }}<0.12$,

- calorimeter isolation $I_{\text {cal }}<0.2$.

- If the primary lepton is a muon then the secondary lepton must be a muon in the CMU, CMU/CMP, CMP, or CMX. Tracks in the CTC which do not extrapolate to the muon chambers but do point to calorimeter towers where energy consistent with 
having come from a minimum ionizing particle has been deposited are also considered (CMIO particles). The following criteria must be satisfied:

$-P_{T}>10 \mathrm{GeV} / c$,

- $E_{\text {had }}<10 \mathrm{GeV} / c^{2}$ for CMU, CMU/CMP, CMP, and CMX muons; $E_{\text {had }}<$ $6 \mathrm{GeV} / c^{2}$ for CMIO muons.

- $E_{\mathrm{cm}}<5 \mathrm{GeV} / c^{2}$ for CMU, CMU/CMP, CMP, and CMX muons; $E_{\mathrm{em}}<2 \mathrm{GeV} / c^{2}$ for CMIO muons.

- Calorimeter isolation $\boldsymbol{I}_{\text {cal }}<0.2$.

- Track stub match $\Delta x<5 \mathrm{~cm}$ for CMU, CMU/CMP, CMP and CMX muons.

$-|\eta|<1.2$ for CMIO muons.

The event is flagged as a $Z$ and removed from the lepton+jet data sample if the primary and secondary lepton are oppositely charged and form an invariant mass between $75 \mathrm{GeV}$ and $105 \mathrm{GeV}$.

\subsubsection{Silicon Vertex Tracker (SVX) Tagging Sample}

We require at least one of the jets in the SVX sample to have a vertex which is displaced from the primary interaction vertex. The algorithm used to identify displaced vertices is called SECVTX, and is described in [23]. The displaced vertex indicates that the jet may have been formed by the decay of a long lived $B$ meson, and is thus an excellent tool for separating the $b$ rich $t \vec{t}$ signal from the $W+$ jet background. 34 events are found to have at least one SVX tagged jet, with an estimated background of $9.2 \pm 1.2$ events [23].

\subsubsection{Soft Lepton Tag (SLT) sample}

In this sample we require an additional lepton to be in the event within a cone of radius $\Delta R \leq 0.4$ of one of the hadronic jets. This lepton is assumed to have been produced in the decay $b \rightarrow l \nu c$, and is thus an indication that the jet was produced by the decay of 


\begin{tabular}{|ccc|}
\hline Variable & Tight cut & Loose cut \\
\hline$E / P$ & $<1.8$ & $<4.0$ \\
$E_{\text {had }} / E_{\text {ern }}$ & $<0.05$ & $<0.055+\frac{0.045 E}{100}$ \\
$\chi_{\text {strip }}^{2}$ & $<10.0$ & Not applied \\
\hline
\end{tabular}

Table 3.1: We list here the differences between the loose and tight selection requirements for electrons.

a B meson. To further reduce the background in this sample we require a fourth jet in the event with $E_{T}>8 \mathrm{GeV} / c^{2}$ and $|\eta| \leq 2.4$. Events with both an SVX tag and an SLT tag are removed from the SLT tag sample so that the SLT sample and SVX sample are orthogonal. There are 14 events in the SLT sample with a background of $6.0 \pm 1.2$ events [26].

\subsubsection{No-tag Sample}

Our last lepton+jet subsample is formed by requiring a fourth jet with $E_{T}>$ $15 \mathrm{GeV} / \mathrm{c}^{2}$ and $|\eta| \leq 2.0$. This sample contains 46 events and has an estimated background of $26 \pm 7.4$ events [26].

\subsection{Dilepton Sample}

The basic signature of a dilepton event is two leptons, missing transverse energy from the neutrinos, and two jets from the $b$ quarks that are produced in the top decay [22]. In the dilepton selection, two categories of lepton are defined: tight and loose. To be considered in the dilepton selection process, an event must include at least one tight lepton, and must include a second lepton which is either tight or loose. An electron or muon which passes all of the selection requirements that are listed in Sections 3.1 or 3.2 is considered to be a tight lepton.

Loose electrons are selected by relaxing the selection requirements on the same variables that are used for tight electrons. The threshold for the $E / P$ cut is increased and a term linear in the electrons energy has been added to the $E_{\text {had }} / E_{\text {em }}$ cut. The $\chi_{\text {strip }}^{2}$ cut 
The Data
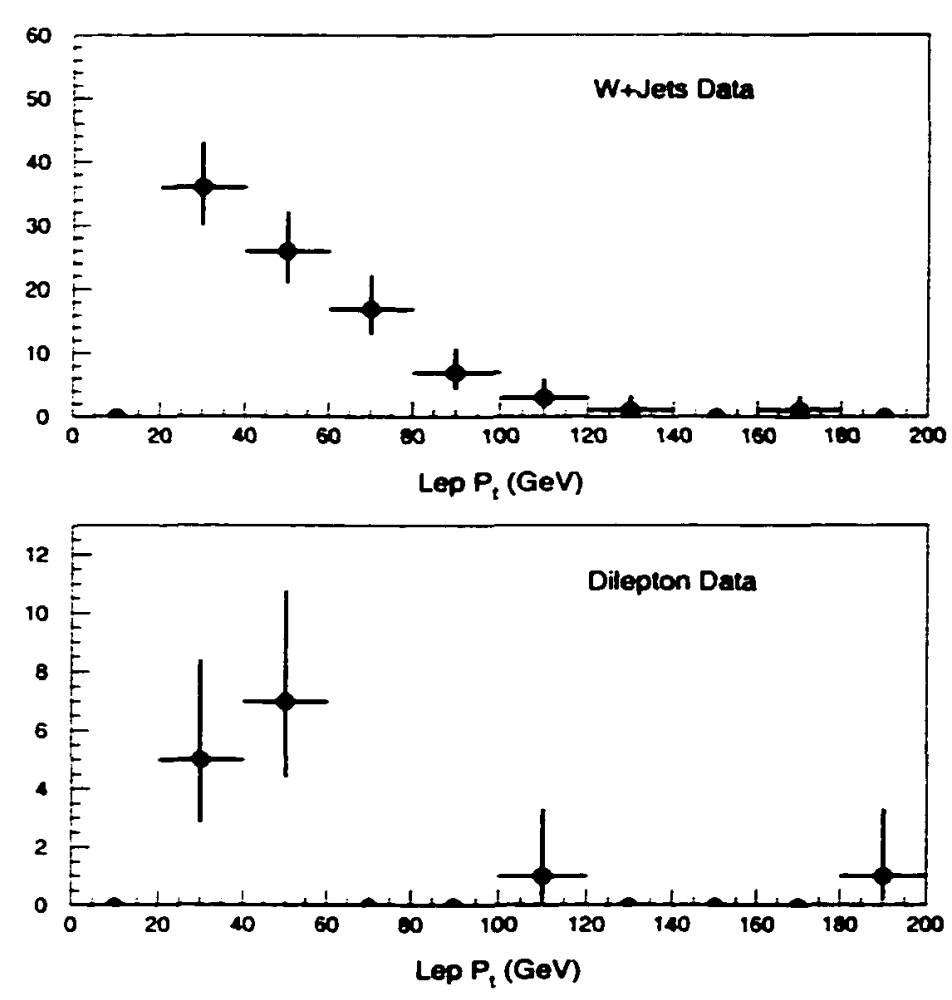

Figure 3.1: The lepton $P_{T}$ distributions for the $1+$ jet and dilepton samples. We shall extract the longitudinal helicity fraction from these distributions by fitting them to negative helicity and longitudinal models using a maximum loglikelihood technique.

is eliminated. See Table 3.1 for a summary of the changes to the selection requirements for loose electrons.

The loose muon category consists of tracks which pass through the CTC and point at a calorimeter tower with energy deposition that is consistent with having come from a muon, but which does not have a matching stub in one of the muon chambers. These muons are also referred to as CMI muons. 


\begin{tabular}{|l|c|c|}
\hline Data Sample & Number of Events & Background Estimate \\
\hline SVX tagged & 34 & $9.2 \pm 1.2$ \\
SLT tagged & 14 & $5.6 \pm 1.2$ \\
Notag & 43 & $23.6 \pm 6.5$ \\
\hline 1+jet total & 91 & $38.3 \pm 6.7$ \\
\hline \hline total leptons & 105 & $39.1 \pm 6.7$ \\
\hline
\end{tabular}

Table 3.2: Observed events and predicted backgrounds for the lepton + jet data subsamples. The lepton + jet entry is the sum of the three entries which precede it.

\subsection{Summary}

These samples have been studied by other CDF analyses and their backgrounds are well understood. The background to the SVX tagged sample is described in [23]. The Notag and SIT samples are described in [26, 27]. We summarize in Table 3.2 the number of events in each sample and the estimated backgrounds. We discuss the shape of the lepton $P_{T}$ spectrum for each of these backgrounds in chapter 4.

In Figure 3.1 we show the lepton $\boldsymbol{P}_{T}$ distributions for all four samples in Run I. Note that our dilepton sample has 14 entries instead of 7 because each event has two leptons which we can use in our fit. 


\section{Chapter 4}

\section{Models for Signal and Background}

In practice it can be quite difficult to relate theoretical predictions to experimental observables. Typically, a single $\mathbf{p} \overline{\mathbf{p}}$ collision results in countless of interactions within the detector, as the particles that are produced in the collision decay into many lighter particles which then interact within the detector components to produce the signature that we recognize as a single event. To calculate the result each of these interactions analytically would be a truly monumental task. Instead, high energy physicists rely on a tool called the "Monte Carlo" calculation to bridge the gap between theory and data.

A Monte Carlo calculation begins with a list of final states for each interaction that is to be modeled, along with the probability for each of these final states to occur. The list of probabilities is drawn from some theoretical model which we are interested in. For each interaction a random number is generated and then compared to the list of probabilities to select one of the final states. For instance, a Monte Carlo which models $t \bar{t}$ production and decay would compare random numbers to the probability densities that describe the distribution of the energy momentum four-vectors of the top and anti-top and their daughter particles. This process is repeated until a satisfactory number of Monte Carlo events has been collected. We can then plot any variables in which we have interest. In principal, the

\footnotetext{
'The Monte Carlo technique relies on a random sampling of the integrals which must be calculated to estimate their value, hence it was named after a city famous for gambling
} 
distribution of any variable that we plot approaches its analytic shape as the number of Monte Carlo events increases.

A Monte Carlo which produces a list of particle four-vectors is called a generator level Monte Carlo, because it generates the particles that we are interested in. Unfortunately, our experimental data is not composed of a clean list of particle four vectors, so we cannot directly compare the output of a generator level Monte Carlo to our experimental data. We must use a second Monte Carlo program which models the response of the CDF detector to the particles which are created in the generator Monte Carlo. We use a program called QFL' $^{\prime}[28,29,30,31,32,33,34]$ in this analysis, because it is good at modelling the response of the calorimeters to hadronic jets. QFL' produces output which mimics the format of real CDF data.

One nice feature of the Monte Carlo technique is that it produces "events" that look like real data. Since the Monte Carlo events are in the same format as the data events experimenters can compare them directly. Competing models can be evaluated against the data if we use their predictions to construct the probability distributions for the Monte Carlo generator.

For this analysis we use many different Monte Carlo generators. To model $t \bar{t}$ production and decay we primarily use the HERWIG [35] Monte Carlo generator. As a cross-check we also generate $t \bar{t}$ with the PYTHIA [36] Monte Carlo generator. Both of these generators begin with a leading order matrix element calculation for the QCD hard scattering process convoluted with the parton distribution functions. They differ in their handling of gluon radiation and the underlying event. In HERWIG the hard scattering is followed by color coherent parton shower evolution, cluster hadronization, and an underlying event based upon data. PYTHIA includes color coherent scattering, but uses string hadronization and an underlying event model based upon multiple parton scattering. In PYTHIA, the initial state and/or final state gluon radiation can be turned off, which is useful for studies of experimental uncertainties caused by inadequate modeling of these effects. To model the backgrounds to our lepton+jet samples we use VECBOS [37], a leading order Monte Carlo 
generator which describes the recoil of a $W$ or $Z$ against quark or gluon jets. In VECBOS, parton shower evolution and hadronization are handled by a routine called HERPRT, which was imported from HERWIG.

\subsection{GGWIG: A Custom HERWIG Monte Carlo Generator}

We use a custom version of the HERWIG Monte Carlo generator to model $t \bar{t}$ production and decay. Our custom version is based upon HERWIG version 5.6. In HERWIG V5.6 the phase space for the decay of the $W$ is properly weighted by the top mass to produce the correct angular distributions for standard model top quark decay but no means is provided to allow a user to change the $W$ helicity to non-standard model values. We use a version of the HERWIG V5.6 routine HWDHQK which was modified by CDF collaborator G. Guillian to allow users complete freedom to set the degree of polarization for both the top quark and the $W$ boson. These modifications are documented in [38]. We refer to the custom HERWIG V5.6 as GGWIG in this thesis, although it is called SPINWIG in some other documents.

\subsection{PYTHIA}

We generate an alternative set of Monte Carlo t $\bar{t}$ events using PYTHIA V5.7. There are a few reasons for us to do this:

- In HERWIG (and GGWIG) it is impossible to turn off initial and/or final state radiation. This can be done in PYTHIA, so we can use our PYTHIA samples to examine systematic uncertainties due to gluon radiation.

- It is important to estimate how our measurement may depend on detailed (an unknown) assumptions of the Monte Carlo model. We can do this if we measure the longitudinal helicity fraction with a PYTHIA $t \overline{\mathrm{t}}$ sample and then compare the re- 


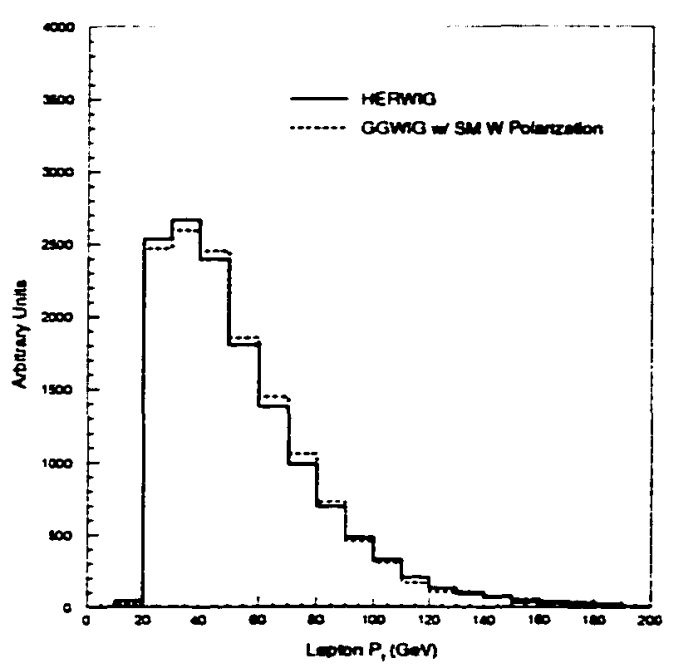

Figure 4.1: Comparison between lepton $P_{T}$ distributions from GGWIG set to $70 \%$ longitudinal $W$ compostion and HERWIG. In both samples the top mass is $175 \mathrm{GeV}$.

sult with that generated using the GGWIG $t \bar{t}$ sample. The Monte Carlo model uncertainty will be related to the difference between the two results.

Unfortunately, we do not have the ability to change the helicity fractions within PYTHIA. To separate our PYTHIA samples into negative helicity and longitudinal templates we resort to a random-weighting technique. We form the angle between the lepton direction in the $W$ rest frame and the $W$ direction in the top rest frame for each event from the PYTHIA GENP bank and then use the helicity angular distributions to determine the probability that the lepton came from a negative helicity $W$. We compare this probability to a random throw between 0 and 1 to create separate helicity distributions. Note that samples generated this way necessarily have lower statistics in the left handed distribution. Additionally, it is impossible to generate positive helicity $\mathbf{W}$ boson this way.

\subsubsection{Background Models}

We also require reliable models for the shape of the lepton $P_{T}$ distribution for each of our backgrounds. For the lepton + jets sample we use VECBOS with $\mathbf{W}+3$ jet matrix elements. VECBOS generates only the $W+$ light jets portion of the lepton + jets 


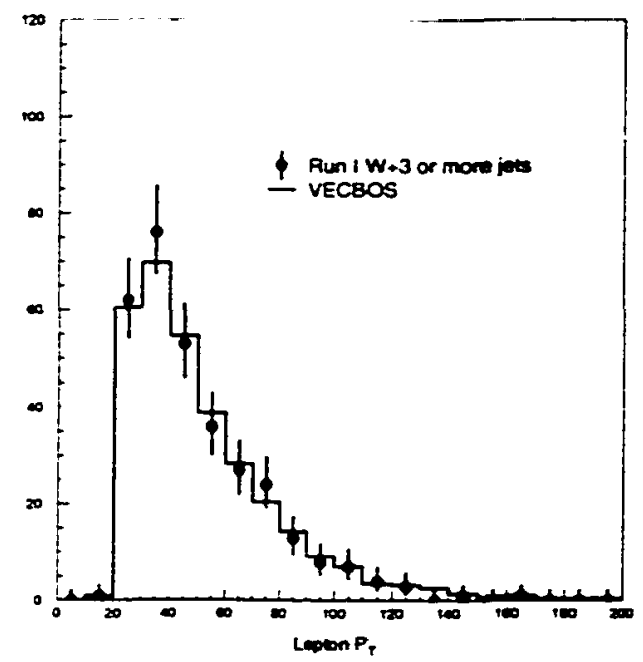

Figure 4.2: Lepton $P_{T}$ distribution from VECBOS with $\mathrm{W}+3$ Jet matrix elements compared with lepton + jet data from run I.

\begin{tabular}{|l|l|c|}
\hline Data Sample & Background Source & Background Prediction \\
\hline$e \mu$ & $Z \rightarrow \tau \tau$ & $0.38 \pm 0.11$ \\
& WW & $0.20 \pm 0.09$ \\
& Fake & $0.16 \pm 0.16$ \\
& b $\bar{b}$ & $0.02 \pm 0.02$ \\
& Total $e \mu$ background & $0.76 \pm 0.21$ \\
\hline$e e, \mu \mu$ & Drell-Yan & $0.61 \pm 0.30$ \\
& $Z \rightarrow \tau \tau$ & $0.21 \pm 0.08$ \\
& Fake & $0.21 \pm 0.17$ \\
& WW & $0.16 \pm 0.07$ \\
& b $\bar{b}$ & $0.03 \pm 0.02$ \\
& Total $e e, \mu \mu$ background & $1.22 \pm \mathbf{0 . 3 6}$ \\
\hline
\end{tabular}

Table 4.1: A table of dilepton backgrounds

background explicitly, but the matrix elements for $W+b \bar{b}$ and $W+$ are identical to the $W$ + light quark elements save for the (negligible) quark masses and it has been shown that VECBOS models well even the non-W portion of the top background [39]. We check that VECBOS models the lepton $\boldsymbol{P}_{T}$ distribution correctly by comparing our VECBOS lepton $P_{T}$ distribution to the lepton $P_{T}$ distribution of the lepton + jet sample from run Ia and Ib in Figure 4.2 and find that the agreement is quite good. 
The background for our current dilepton selection cuts comes from three primary sources, WW, $Z \rightarrow \tau \tau$, and lepton + jets events where a jet fakes a lepton. We use the following models:

WW This background is simulated with PYTHIA.

$Z \rightarrow \tau \tau$ For this background we use the ISAJET Monte Carlo.

fakes We use lepton + jets data from run I to compute this background distribution. We first select a sample of events which has a $20 \mathrm{GeV}$ lepton, three or more $10 \mathrm{GeV}$ jets, and $F_{T}$ greater than $20 \mathrm{GeV}$. We then randomly assign one of the jets to be a lepton and then check to see if the event passes dilepton cuts.

We generate the distribution of lepton $\boldsymbol{P}_{T}$ from each source, and then combine them with appropriate weights to form the dilepton background distribution. In Table 4.1 we reproduce a summary of dilepton background estimates which is found in [22]. This Table contains an entry for $\mathbf{b} \bar{b}$ events, $a$ fourth source of backgrounds to the e $\mu$ dilepton sample. We ignore this background because it is very small compared to the other background components.

Table 4.1 also contains a listing of backgrounds for the same flavor (ee and $\mu \mu$ ) dilepton events. The background models we have discussed can account for only $50 \%$ of this background, the rest is Drell-Yan. We do not have a good model for the lepton $P_{T}$ distribution in these events. Thus, we shall leave the ee and $\mu \mu$ events out of the analysis. These events will enhance our data sample by only 4 leptons while adding $2.44 \pm .72$ background leptons, so we anticipate no loss of precision for leaving them out of the data sample. 


\section{Chapter 5}

\section{Measuring the Helicity of the W Boson}

The helicity of $W$ bosons which are produced in top quark decays can be analyzed via the sequential decay of the $W$ to a charged lepton and a neutrino, because the $V-A$ nature of the weak coupling induces a correlation between the $W$ spin and the decay angle of the charged lepton in the top rest frame. This correlation translates into the lab frame as a helicity dependence in the transverse momentum spectrum of the charged lepton. In this chapter we use a minimum negative $\log$-likelihood fit of the charged lepton $P_{T}$ spectrum in top decays to the sum of expectations for the three $W$ helicities plus backgrounds to measure the helicity of the $W$ in top decays. As we shall demonstrate below, we do not have enough statistics for a simultaneous fit for all three helicity fractions. What we shall do instead is to perform two likelihood fits. In the first we shall assume, as per standard model predictions, that no positive helicity $W$ bosons are present, and we shall then fit to find the fractions of negative helicity and longitudinal $W$ bosons in top decays. In the second fit we shall assume that the standard model prediction for the fraction of $W$ bosons produced in the longitudinal mode is correct and then fit for the fractions of negative and positive helicity $\mathbf{W}$ bosons. 


\subsection{Likelihood Function}

We use an unbinned minimum negative log-likelihood fit to extract the fraction of top quarks which decay to longitudinal $\mathbf{W}$ bosons $\left(\mathcal{F}_{0}\right)$. A log-likelihood is constructed by taking the logarithm of the probability that a given set of parameters can generate an observed ensemble of events. For our purposes this probability takes the form

$$
\mathcal{P}\left(x_{i} ; \mathcal{F}_{0}, \beta\right)=\prod_{j=1}^{N_{S}}\left[\mathcal{P}_{j}^{B}\left(\beta_{j}\right) \prod_{i=1}^{N_{E}^{j}} \mathcal{P}_{j}^{S}\left(x_{i} ; \mathcal{F}_{0}, \beta_{j}\right)\right]
$$

In equation 5.1 the function $\mathcal{P}_{j}^{B}\left(\beta_{j}\right)$ represents the probability that the background fraction of data sample $\mathrm{j}$ is $\boldsymbol{\beta}_{j}$ and the function $\mathcal{P}_{j}^{S}\left(x_{i} ; \mathcal{F}_{0}, \beta_{j}\right)$ is the probability of observing an event with lepton $\boldsymbol{P}_{T}$ of $\boldsymbol{x}_{i}$ given a longitudinal fraction $\mathcal{F}_{0}$ and a background fraction $\boldsymbol{\beta}_{j} . \boldsymbol{N}_{S}$ is the number of distinct data samples used in the analysis, and $N_{E}^{j}$ is the number of events in each of these samples. The functions $\mathcal{P}^{S}$ are simply the linear sum of the individual probabilities of observing a negative helicity, longitudinal, or background event at a lepton $P_{T}$ of $x_{i}$ weighted by the parameters $\mathcal{F}_{0}$ and $\beta_{j} ;$ e.g.,

$$
\mathcal{P}_{j}^{S}\left(x_{i} ; \mathcal{F}_{0}, \beta_{j}\right)=\left(1-\mathcal{F}_{0}-\mathcal{F}_{+}\right)\left(1-\beta_{j}\right) T_{j}^{(-)}+\mathcal{F}_{0}\left(1-\beta_{j}\right) T_{j}^{(0)}+\mathcal{F}_{+}\left(1-\beta_{j}\right) T_{j}^{(+)}+\beta_{j} T_{j}^{B}
$$

The functions $T^{(-)}\left(x_{i}\right), T^{(0)}\left(x_{i}\right), T^{(+)}\left(x_{i}\right)$ and $T^{B}\left(x_{i}\right)$ describe the shape of the lepton $P_{T}$ spectra for all components which we expect to see in the data. The functions $T^{(-)}\left(x_{i}\right)$, $T^{(0)}\left(x_{i}\right)$, and $T^{(+)}\left(x_{i}\right)$ describe the lepton $P_{T}$ spectra for leptons from top events where the $\mathrm{W}$ boson is negative helicity, longitudinal, or positive helicity, respectively. The function $T^{B}\left(x_{i}\right)$ describes the lepton $P_{T}$ spectrum in the background. We call these functions "shape templates." We construct the shape templates for $t \bar{t}$ events from the GGWIG Monte Carlo 4.1 by fitting the binned distributions to the product of an exponential and a polynomial. This functional form was chosen because it models very well the distributions which it must fit, as can be seen in Figure 5.1. The shape templates are normalized to an area of 1 in the region of importance to our fit $\left(20 \mathrm{GeV} / c<P_{T}<200 \mathrm{GeV} / c\right)$. Note that the parameter $\mathcal{F}_{-}$does not appear in Equation 5.2. This is because we apply the requirement 
that the three helicity fractions sum to one. Thus we obtain the fraction $\mathcal{F}_{-}$after the fit from the relation $\mathcal{F}_{-}=1-\mathcal{F}_{0}-\mathcal{F}_{+}$.

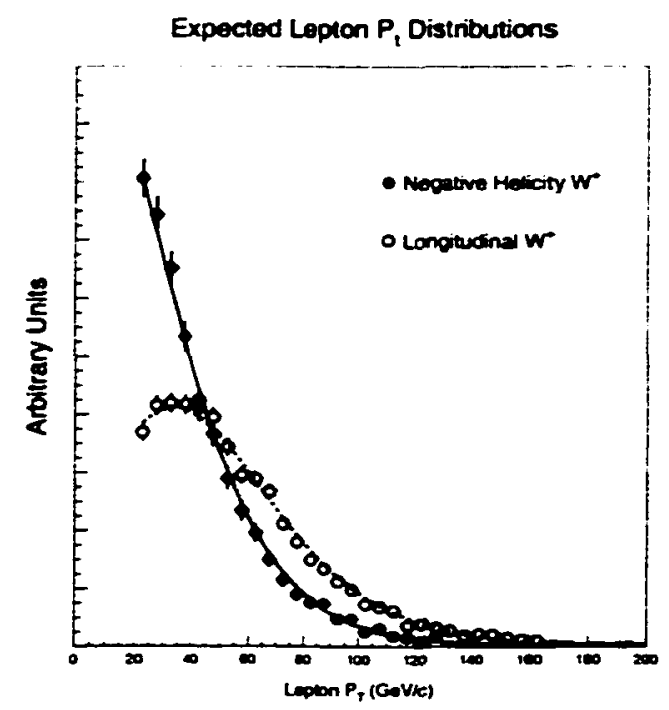

Figure 5.1: The circles in this plot represent the $\boldsymbol{P}_{T}$ distributions for leptons from negative helicity and longitudinal $W$ Bosons, as predicted by GGWIG. The smooth lines are the best fit to the Monte Carlo distributions using the product of an exponential and a polynomial function.

The function $\mathcal{P}_{j}^{B}\left(\beta_{j}\right)$ allows us to include in our likelihood our knowledge about the background contents in each of the subsamples as a constraint. This is necessary because our fit does not have enough statistical power to determine the size of the background component without help from outside predictions. The form of $\mathcal{P}_{j}^{B}\left(\beta_{j}\right)$ depends upon the data sample being considered. In the lepton + jets data our backgrounds are computed as a fraction of the size of the sample, so use a Gaussian probability distribution to constrain $\beta_{j}$ directly. A Poisson constraint on the number of events in the lepton+jet samples would have no effect on the measurement because it has no functional dependence on $\beta_{j}$, so we do not include such a constraint. In the dilepton sub-sample we have a direct prediction for the number of background events that we should observe. In this case we allow the sum of the signal and background events to fluctuate, but constrain them with a Poisson probability density function $P\left(N, n_{s}+n_{b}\right)$ in $N$ with mean $n_{s}+n_{b}$, where $N$ is the number of events 
observed in the dilepton sub-sample and $n_{,}$and $n_{b}$ are the expectation value of the number of signal and background events in the sample, respectively. We further constrain $n_{b}$, the number of background events, with a Gaussian probability distribution of mean $\left\langle\boldsymbol{n}_{b}\right\rangle$ and width $\sigma_{n}$. In the lepton+jet sub-samples, $\beta_{j}$ is a parameter in the likelihood minimization, whereas in the dilepton sub-sample $n_{b}$ and $n_{b}$ are parameters in the likelihood minimization and $\beta_{j}$ is computed from the relation $\beta_{j}=n_{b} /\left(n_{b}+n_{b}\right)$.

To form our negative $\log$-likelihood we simply take the logarithm of $\mathcal{P}\left(x_{i} ; \mathcal{F}_{0}, \beta\right)$ and multiply by negative 1 . We use the Cernlib package MINUIT to find the minimum value of our negative $\log$ likelihood as a function of the helicity fractions. We determine the statistical uncertainty in the fit parameters by varying them until the negative log-likelihood increases by 0.5 .

\subsection{Verification of the Likelihood Technique}

\subsubsection{Consistency and Sensitivity}

We use a Pseudo-Experiment technique to verify the consistency of our likelihood minimization, to estimate the statistical sensitivity of our analysis, and as a tool to aid our investigation of the systematic uncertainties of this analysis. The basic procedure is to generate a random distribution of lepton $\boldsymbol{P}_{T}$ values with fixed normalization, using a set of templates as weights and then apply our likelihood technique to this distribution to extract from it $\mathcal{F}_{0}$. We can use different sets of templates to generate our "data" than we use to analyze it. This will prove useful in Chapter 6 where we evaluate our systematic uncertainties.

We first apply this technique to check consistency and determine the sensitivity we can expect from our plan to use a dilepton and combined lepton + jets sample. We generate 1000 experiments with 94 lepton + jets entries and 14 dilepton entries (because our 7 dilepton events each have 2 leptons). We use our analysis templates to generate these events with a longitudinal helicity fraction of .75. The number of background events 

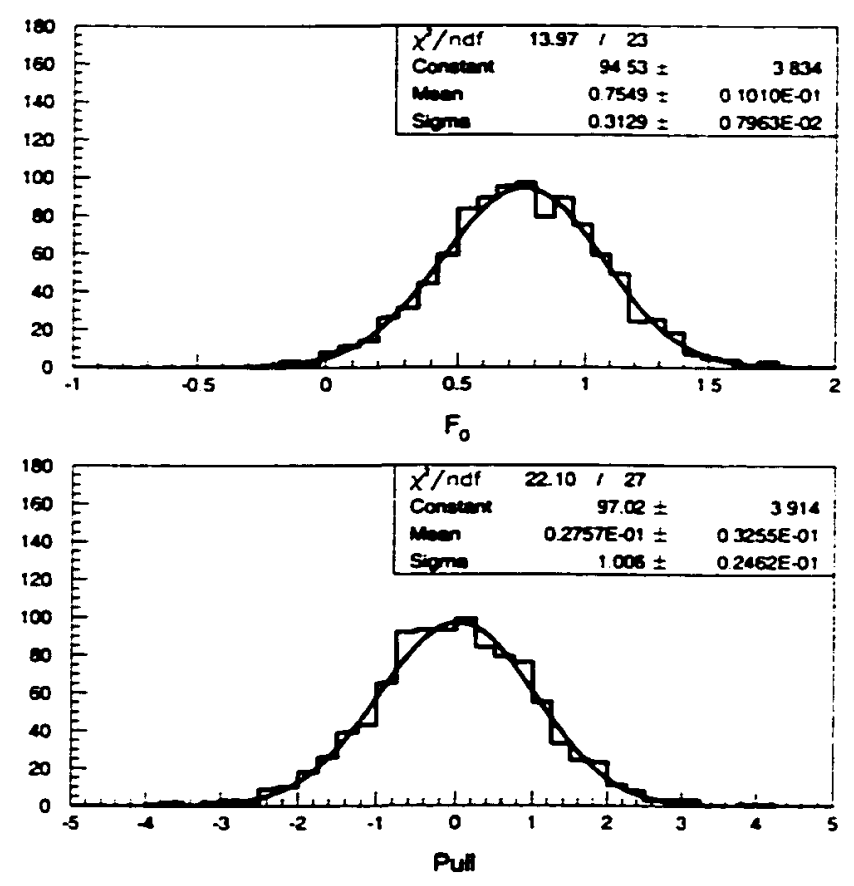

Figure 5.2: Pseudo-experiment study of the maximum likelihood procedure. Top plot is distribution of returned values for input $\mathcal{F}_{0}=0.70$, bottom plot is the distribution of the pulls.

in these templates varies as a Poisson random variable whose mean varies according to a Gaussian distribution. The total number of events is the same for each pseudo-experiment. In Figure 5.2 we show the distribution of $\mathcal{F}_{0}$ values and their corresponding pulls which are returned by this exercise. The $\mathcal{F}_{0}$ values are fit very well by a Gaussian with a mean of .75 and a width of .31 . We can draw two conclusions from this plot.

- Our likelihood technique does not bias our result (eg. we get out what we put in, on average).

- We can expect our fit to the data to return a statistical uncertainty of about \pm .3 .

The second conclusion derives from the width of the Gaussian fit to the $\mathcal{F}_{0}$ values returned by the pseudo-experiments and is supported by the fact that the pull distribution for this exercise (also shown in Figure 5.2) has a width very close to one. 
Note that in Figure 5.2 some pseudo-experiments returned (unphysical) values of $\mathcal{F}_{0}$ which are greater than 1 or less than 0 . This is because we do not place limits on $\mathcal{F}_{0}$ in our likelihood fit. These unphysical results do not worry us, because the Gaussian distributions returned by our pseudo-experiments demonstrate that this analysis is robust and we have thus decided not to place any limits on the parameters in our likelihood fit.

\subsubsection{Investigation of the Background Fraction Term in the Likelihood}

To test the sensitivity of our analysis to the form of the background constraint we repeat the pseudo-experiment analysis for two extreme modifications: 1) we fix the background to its predicted value, and 2) we constrain the background with a Gaussian that has twice the width of the uncertainty on the background. The $\mathcal{F}_{0}$ 's and their associated pulls are shown in Figure 5.3. In both cases the mean of the $\mathcal{F}_{0}$ distributions are consistent with the input value of $\mathcal{F}_{0}$ and the width of the distributions are consistent with the width which we observed using our nominal background shape functions. There is pattern to the returned widths, the smallest value $(0.307 \pm 0.007)$ is returned by the fit in which the background is fixed and the largest value $(0.315 \pm 0.007)$ is returned by the fit with the doubled Gaussian width. This is as one would expect, since the fixed background gives the likelihood less latitude in determining the most likely value of $\mathcal{F}_{0}$ than our nominal function does and the doubled-Gaussian function gives more latitude. In any case, the difference is negligible compared to other uncertainties in the analysis.

\subsection{Acceptance Bias}

Longitudinal $\mathrm{W}$ bosons produce a harder lepton $\boldsymbol{P}_{T}$ distribution than negative helicity W's and thus are more likely to be accepted by the lepton $P_{T}$ cut which is applied to select top candidates. This implies that our procedure overestimates $\mathcal{F}_{0}$. In general, the acceptance biases for the lepton + jet and dilepton samples need not be the same, because in the lepton + jet sample only the helicity of the leptonically decaying $W$ is relevant, while 

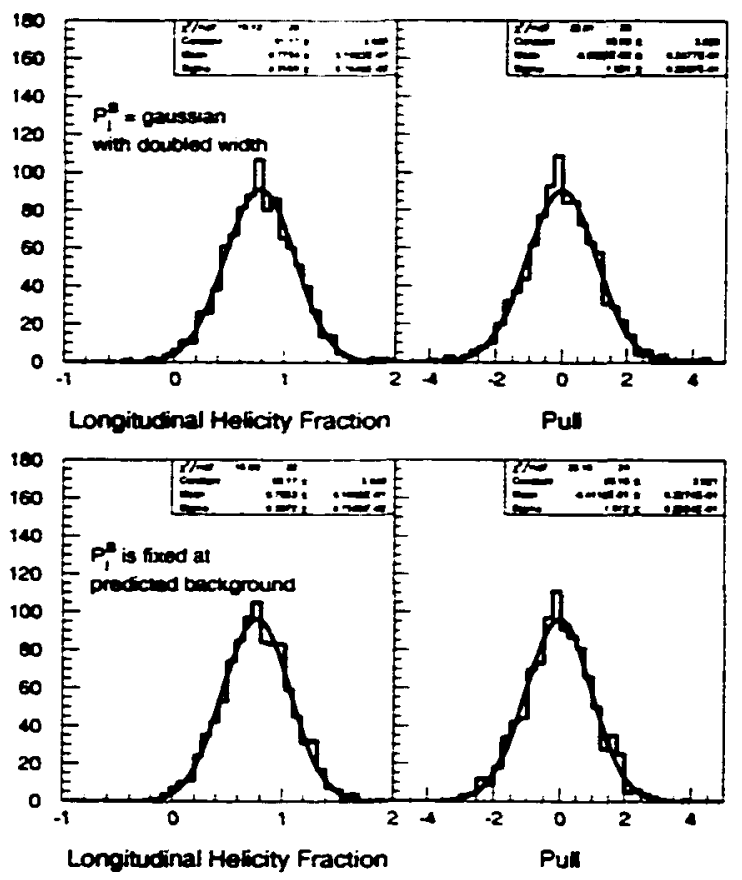

Figure 5.3: Checks of the sensitivity to the background assumptions.

in the dilepton sub-sample both W's can contribute to the acceptance bias. In the following sections we will derive separate corrections for the lepton + jet and dilepton samples and then combine them to obtain a correction for the simultaneous fit.

\subsubsection{Acceptance Correction for Lepton + Jets}

Throughout this discussion it is assumed that the hadronization of quarks produced in $W$ decays wipes out effects that the helicity of the hadronically decaying $W$ boson may have on acceptance. Because of this we need only study the acceptance bias induced by the leptonically decaying $\mathbf{W}$ boson. Thus, it is straightforward to develop a correction function for the lepton + jets sample which takes our fit fraction $\mathcal{F}_{0}^{\text {fit }}$ to the correct value.

We start by writing down a function for the measured longitudinal fraction $\left(\mathcal{F}_{0}^{\text {fit }}\right)$ as a function of the true longitudinal fraction $\left(\mathcal{F}_{0}\right)$ and the acceptances for events with either 
negative helicity of longitudinal $W$ bosons in them. ( $A_{-}$and $A_{0}$ ).

$$
\mathcal{F}_{0}^{\text {fit }}=\frac{\mathcal{L} \sigma_{\mathrm{t} \overline{\mathrm{t}}} A_{0} \mathcal{F}_{0}}{\mathcal{L} \sigma_{\mathrm{t} \overline{\mathrm{t}}} \boldsymbol{A}_{0} \mathcal{F}_{0}+\mathcal{L} \sigma_{\mathrm{t} \overline{\mathrm{t}}} A_{-}\left(1-\mathcal{F}_{0}\right)}
$$

It is a simple matter of algebra to solve equation 5.3 for $\mathcal{F}_{0}$.

$$
\mathcal{F}_{0}=\frac{\boldsymbol{R}_{(-/ 0)} \mathcal{F}_{0}^{\text {fit }}}{1-\mathcal{F}_{0}^{\text {fit }}\left(1-\boldsymbol{R}_{(-/ 0)}\right)}
$$

In equation 5.4 we have divided through the numerator and denominator by the longitudinal acceptance so that we can express $\mathcal{F}_{0}$ as a function of the ratio $\left(\boldsymbol{R}_{(-/ 0)}\right)$ of negative helicity to longitudinal acceptances. Equation 5.4 is non-linear and will in general cause the uncertainties calculated for $\mathcal{F}_{0}^{\text {fil to change. }}$

To derive the factor $\boldsymbol{R}_{(-/ 0)}$, we generate GGWIG samples which contain only negative helicity W's or only longitudinal W's and then apply our selection requirements and compute the acceptance for each sample separately. In particular, we select events from our negative helicity and longitudinal GGWIG samples which pass the same acceptance cuts as the data, which are outlined in Chapter 3. The number of Monte Carlo events which satisfy our acceptance criteria is summarized in Table 5.1 .

We next apply to the events that pass the selection criteria the lepton scale factors which are used in both lepton + jets cross-section analysis, CMU/CMP $=0.943, \mathrm{CMX}$ $=0.940$, and $\mathrm{CE}=0.917$. We do not apply b-tagging efficiencies to our acceptance calculations because we are ultimately interested only in the ratio of the negative helicity and longitudinal acceptances and so multiplicative factors which are common to both will cancel out. However, we do multiply by the $Z$ vertex efficiency (0.95), as per [40]. The acceptance for top's that decay into negative helicity $W$ bosons is calculated to be 0.0867 and the acceptance for tops which decay into longitudinal $W$ bosons is calculated to be 0.1127. This results in a ratio $R_{(-/ 0)}=0.769 \pm 0.029$. The uncertainty on $R_{(-10)}$ includes only the statistical uncertainty on the calculation of $A_{-}$and $A_{0}$. We defer the discussion of the systematic uncertainty on $R_{(-/ 0)}$ to Section 6.4 .

We can check our procedure by comparing the average of these two acceptances to the $t \bar{t}$ acceptance found in the cross section analysis [40], which is calculated using standard 


\begin{tabular}{|l|c|c|c|}
\hline \hline MC Sample & GGWIG & GGWIG & Herwig \\
Longitudinal Fraction & $0 \%$ & $100 \%$ & $70 \%$ \\
Top Mass Used & $175 \mathrm{GeV}$ & $175 \mathrm{GeV}$ & $175 \mathrm{GeV}$ \\
MC Version & V5.6 & V5.6 & V5.6 \\
Number of Gen. Events & 22,164 & 20,714 & 105,260 \\
\hline \hline \# of Events which pass cuts & 2174 & 2640 & 12,695 \\
\hline Electrons & 1015 & 1159 & 5739 \\
Muons & 1159 & 1481 & 6956 \\
CMU/CMP & 895 & 1169 & 5430 \\
CMX & 264 & 311 & 1522 \\
\hline Acceptance & 0.0867 & 0.1127 & 0.1066 \\
\hline \hline
\end{tabular}

Table 5.1: Number of events which satisfy selection cuts in our acceptance calculation. The third column is reproduced from Ref [40] for the sake of comparison.

Herwig to be 0.1066 . We must calculate the weighted average using the fractions of each sort of helicity expected in top decays. When we apply our likelihood technique to standard Herwig we extract a longitudinal fraction of $0.78 \pm 0.02$. If we use this fraction to weight our longitudinal acceptance and its complement to weight the negative helicity acceptance then we obtain an average total acceptance of 0.1070 , in excellent agreement with expectations. The uncertainty due to Monte Carlo statistics on the top acceptance calculation which is quoted in $[40]$ is $2 \%$.

\subsubsection{Acceptance Correction for Dileptons}

To compute an acceptance correction for the dileptons we must modify the procedure developed for the lepton + jets sample to account for the fact that we have two leptons in each event. We must divide the dileptons into three categories of events: those with two longitudinal W's, those with one longitudinal $W$ and one negative helicity $W$, and events with two left handed W's. The probability that a given $t \bar{t}$ event will fall into a particular helicity category depends upon $\mathcal{F}_{0}$ and is given in equation 5.5 , where $P_{00}$ is the probability for both W's to be longitudinal, $P_{0-}$ is the probability that one will be longitudinal and 
the other negative helicity, and $P_{--}$is the probability that both will be negative helicity.

$$
\begin{aligned}
& P_{00}=\mathcal{F}_{0}^{2} \\
& P_{0-}=2 \mathcal{F}_{0}\left(1-\mathcal{F}_{0}\right) \\
& P_{--}=\left(1-\mathcal{F}_{0}\right)^{2}
\end{aligned}
$$

We use these probabilities to calculate $\mathcal{F}_{0}^{\text {fit }}$ for dilepton events as a function of $\mathcal{F}_{0}$ and the acceptances for the three dilepton helicity categories $\left(A_{00}, A_{0_{-}}\right.$, and $\left.A_{--}\right)$in equation 5.6.

$$
\mathcal{F}_{0}^{\text {fit }}=\frac{2 A_{00} P_{00}+A_{0-} P_{0-}}{2 A_{00} P_{00}+2 A_{0-} P_{0-}+2 A_{--} P_{--}}
$$

Inverting equation 5.6 requires us to solve a quadratic equation. Only the negative solution of the quadratic equation produces physically reasonable values of $\mathcal{F}_{0}$, we show it in equation 5.7.

$$
\mathcal{F}_{0}=\frac{\left.2 \mathcal{F}_{0}^{\text {fit }} A_{--}-A_{0-}\left(2 \mathcal{F}_{0}^{\text {fit }}-1\right)-\sqrt{A_{0-}^{2}\left(2 \mathcal{F}_{0}^{\text {fit }}-1\right)^{2}+4 A_{00} A_{--}\left(\mathcal{F}_{0}^{\text {fit }}-\mathcal{F}_{0}^{\text {fit }}{ }^{2}\right.}\right)}{2 A_{00}\left(\mathcal{F}_{0}^{\text {fit }}-1\right)-2 A_{0-}\left(2 \mathcal{F}_{0}^{\text {fit }}-1\right)+2 A_{--} \mathcal{F}_{0}^{\text {fit }}} .
$$

\begin{tabular}{|l|c|c|}
\hline Helicity Category & Acceptance & Probability (given $\left.\mathcal{F}_{0}=0.70\right)$ \\
\hline \hline Two longitudinal W's $\left(\boldsymbol{A}_{0}\right)$ & $(0.87 \pm 0.02) \%$ & $49 \%$ \\
One longitudinal W $\left(\boldsymbol{A}_{0_{-}}\right)$ & $(0.77 \pm 0.02) \%$ & $42 \%$ \\
No longitudinal W's $\left(\boldsymbol{A}_{--}\right)$ & $(0.62 \pm 0.02) \%$ & $9 \%$ \\
\hline Weighted Average & $(0.808 \pm 0.014) \%$ & \\
Standard Herwig & $(0.784 \pm 0.025) \%$ & \\
\hline
\end{tabular}

Table 5.2: Acceptances for the three helicity categories in dilepton events.

To compute the acceptances for the dilepton helicity categories we first generate large GGWIG samples for each category and then apply the same techniques to these samples which were used in the dilepton cross-section analysis to compute the acceptance. This procedure is outlined in [22]. We list the acceptances for each helicity category in Table 5.2. In this table the probability printed in column 3 is the probability that a $t \bar{t}$ event will fall into the helicity category, assuming that $\mathcal{F}_{0}$ is 0.70 , as computed via equation 5.5 . Also included in Table 5.2 are the average of the three acceptances, weighted by the probabilities 
in column 3, and the acceptance for dileptons computed for the dilepton analysis in [22]. Errors in Table 5.2 are statistical only.

\subsubsection{Computing Acceptance Corrections with Pythia}

We intend to use Pythia Monte Carlo to examine two important systematic uncertainties: Monte Carlo generator dependence and the effects of gluon radiation. To effectively test these systematic uncertainties it is necessary that we derive a set of acceptance corrections from Pythia MC. Unfortunately, it is not possible to generate Pythia samples with W's in arbitrary helicity states. We solve this problem by using the random weighting technique described in Section 4.2 to separate our lepton + jet Pythia samples into two subsamples containing negative helicity W's or longitudinal W's, and our dilepton Pythia samples into the three dilepton helicity categories described in Section 5.3.2.

To compute the acceptances for each of these subsamples we must know how many events existed in each of them prior to the analysis cuts. We know how many events were generated to produce the original samples, and we know that in Pythia with a top mass of $175 \mathrm{GeV}, \mathcal{F}_{0}=0.70$. This means that the number of events in the longitudinal lepton + jet sample prior to analysis cuts is 0.7 times the total number of events generated in the lepton + jet sample. The fractions used to determine the number of events in the dilepton subsamples are given in Table 5.2. We list the Pythia acceptances in Table 5.3. Included in this table are the results for Pythia with and without initial and final state radiation. In the dilepton samples turning the radiation off did not have a statistically significant effect. In the lepton + jet samples turning off the initial state radiation reduced the acceptance for both helicity states and turning off final state radiation increased acceptance for both helicities. The ratio between the acceptances of the two helicity states did not change significantly when either type of radiation was turned off. 


\begin{tabular}{|c|c|c|c|}
\hline & Normal Pythia & $\begin{array}{c}\text { Pythia without } \\
\text { Initial State } \\
\text { Radiation } \\
\end{array}$ & $\begin{array}{c}\text { Pythia without } \\
\text { Final State } \\
\text { Radiation } \\
\end{array}$ \\
\hline $\begin{array}{l}\text { Dilepton Acceptances } \\
A_{00} \\
A_{0-} \\
A_{--}\end{array}$ & $\begin{array}{l}(0.82 \pm 0.03) \% \\
(0.67 \pm 0.03) \% \\
(0.46 \pm 0.05) \%\end{array}$ & $\begin{array}{l}(0.79 \pm 0.03) \% \\
(0.66 \pm 0.03) \% \\
(0.58 \pm 0.06) \% \\
\end{array}$ & $\begin{array}{l}(0.88 \pm 0.03) \% \\
(0.74 \pm 0.03) \% \\
(0.60 \pm 0.06) \%\end{array}$ \\
\hline $\begin{array}{l}\text { Lepton }+ \text { jet Acceptances } \\
A_{-} \\
A_{0} \\
R_{(-10)}\end{array}$ & $\begin{array}{c}(8.38 \pm 0.17) \% \\
(10.28 \pm 0.13) \% \\
0.815 \pm 0.019\end{array}$ & $\begin{array}{c}(7.92 \pm 0.17) \% \\
(9.91 \pm 0.12) \% \\
0.799 \pm 0.020\end{array}$ & $\begin{array}{c}(8.91 \pm 0.19) \% \\
(10.97 \pm 0.13) \% \\
0.812 \pm 0.020\end{array}$ \\
\hline
\end{tabular}

Table 5.3: Helicity dependent acceptances for Pythia Monte Carlo. Errors are statistical.

\subsubsection{Combining the Corrections}

We combine the two acceptance corrections which we have calculated by averaging them, weighted by the number of leptons in each sample. In Figure 5.4 we plot the dilepton and lepton + jet corrections as a function of $\mathcal{F}_{0}$. Note that the two acceptance corrections are quite similar in magnitude. This hints that we are unlikely to introduce a significant uncertainty into our analysis via our procedure for combining the two corrections, because all reasonable combinations of the two corrections will be close to both of them. In fact, we do not explicitly include the combined correction in Figure 5.4 because it is indistinguishable from the lepton + jet correction on the scale used for this plot.

We use pseudo-experiments to determine whether our average correction works properly. First we use equations 5.3 and 5.6 to compute the value of $\mathcal{F}_{0}^{\text {fit }}$ which we should observe in the lepton + jet and dilepton samples if $\mathcal{F}_{0}=0.70$. We find that for the lepton + jet sample $\mathcal{F}_{0}^{\text {fit }}=0.75$ and for the dilepton sample $\mathcal{F}_{0}^{\text {fit }}=0.73$. We use these values as the input helicities to generate 1000 pseudo-experiments with 91 lepton + jet events and 7 dilepton events, as per Section 5.2.1. We perform the log-likelihood fit and then apply the combined acceptance correction to the mean of this distribution and the result is $\mathcal{F}_{0}=0.695 \pm 0.009$. 


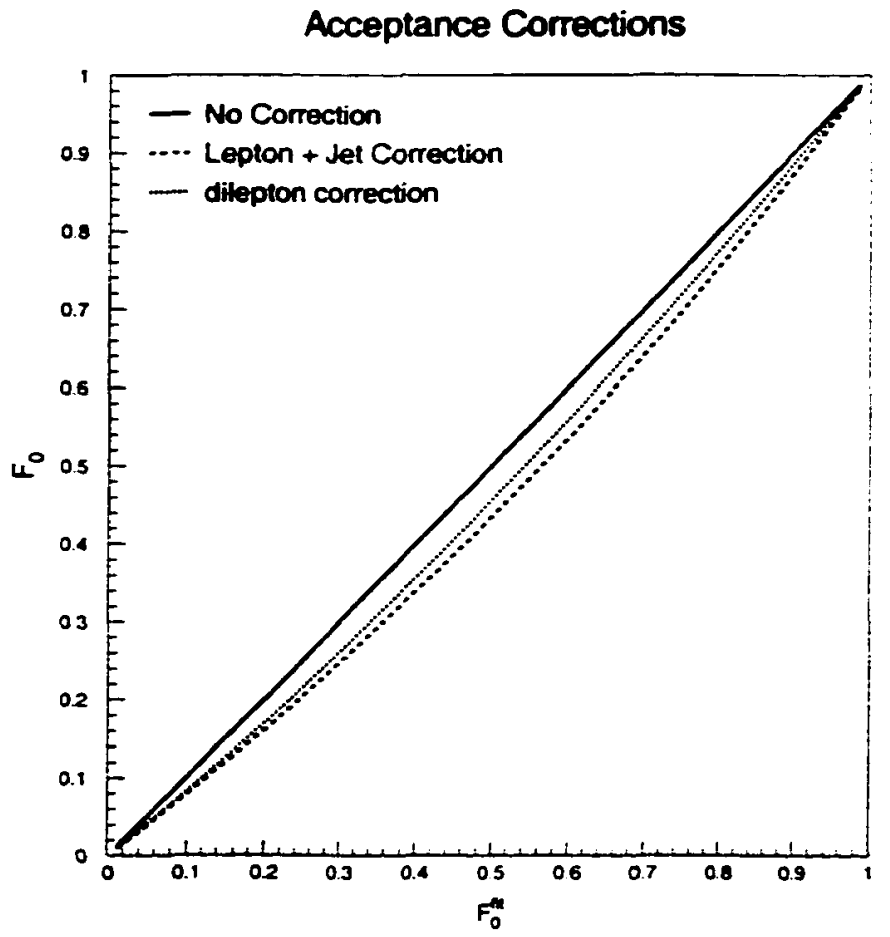

Figure 5.4: Here we show the acceptance corrections for both the lepton+jets and dilepton samples as a function of $\mathcal{F}_{0}^{\text {fit }}$. We do not show the combined correction because it is so close to the lepton + jet correction that they cannot be distinguished from one another on the scale of this figure.

Thus, our combined correction does obtain the original input helicity. This should come as no surprise, because the two corrections are similar in magnitude.

The outcome of these considerations is that we have a procedure to correct the

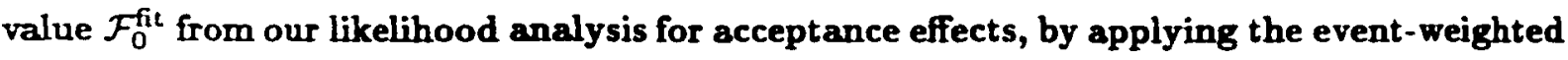
average of Eqn. 5.4 and Eqn. 5.7 with $\boldsymbol{R}_{(-/ 0)}=0.769$ and the dilepton acceptances given in Table 5.2. We discuss the systematic uncertainty introduced by the acceptance correction in Sec 6.4. The acceptance correction modifies the statistical uncertainty on $\mathcal{F}_{0}$, this is discussed in Sec 6.4. 


\subsection{Measurement of $\mathcal{F}_{0}$}

Now that we have established a fit procedure it is time to apply it to our data. We maximize the likelihood established in Equation 5.1 simultaneously for the three lepton + jet samples and the dilepton sample. In this minimization we constrain the background fraction with a Gaussian term in the likelihood, as discussed in Section 5.2.1. In Figure 5.5 we compare the best fit shape with the data in both the lepton + jet and dilepton samples. The result of this fit is $.93_{-.32}^{+.32}$. This uncertainty is consistent with the uncertainties that we expected after performing our pseudo-experiment tests in Section 5.2.1. A visual inspection of Figure 5.5 indicates that our fit models the shape of the Lepton + Jets sample very well. The fit in the dilepton sample is statistically challenged but is also a good fit. It would be instructive to obtain a measure of fit quality that is more quantitative than a visual inspection. Unfortunately, the log-likelihood function cannot be interpreted as a measure of the goodness- of-fit in the same manner as a $\chi^{2}$ likelihood. What we do instead is run 1000 pseudo-experiments and compare the negative log-likelihood values extracted from them to that which is returned by our fit to the data. We find that our log likelihood is smaller than that returned by $31 \%$ of the pseudo-experiments. We call this our "fit quality".

Note that our best fit result must be corrected according to the prescription in Sec 5.3.4 to obtain the "true" value of $\mathcal{F}_{0}$. We use standard techniques [41] to propagate the uncertainty on $\mathcal{F}_{0}^{\text {fit }}$ over to $\mathcal{F}_{0}$, and obtain $\mathcal{F}_{0}=0.91_{-.37}^{+.37}$.

It is interesting to fit each of our data subsamples individually to find out where our statistical power is coming from and to check whether there are any strange features in them which need to be investigated. We summarize all the fit results in Table 5.4. In addition to fit results, we include the fit quality and expected sensitivity for each sample. We include the acceptance corrected helicity fraction for all samples except for Notag and SLT samples. These two samples return unphysical helicity fractions for which the correction is not defined. Note that in all cases the statistical uncertainty returned by our fit to the data is consistent with the sensitivity which we predict via pseudo-experiments.

The dilepton sample has a rather poor fit quality, $5.6 \%$. The poor quality of the fit 


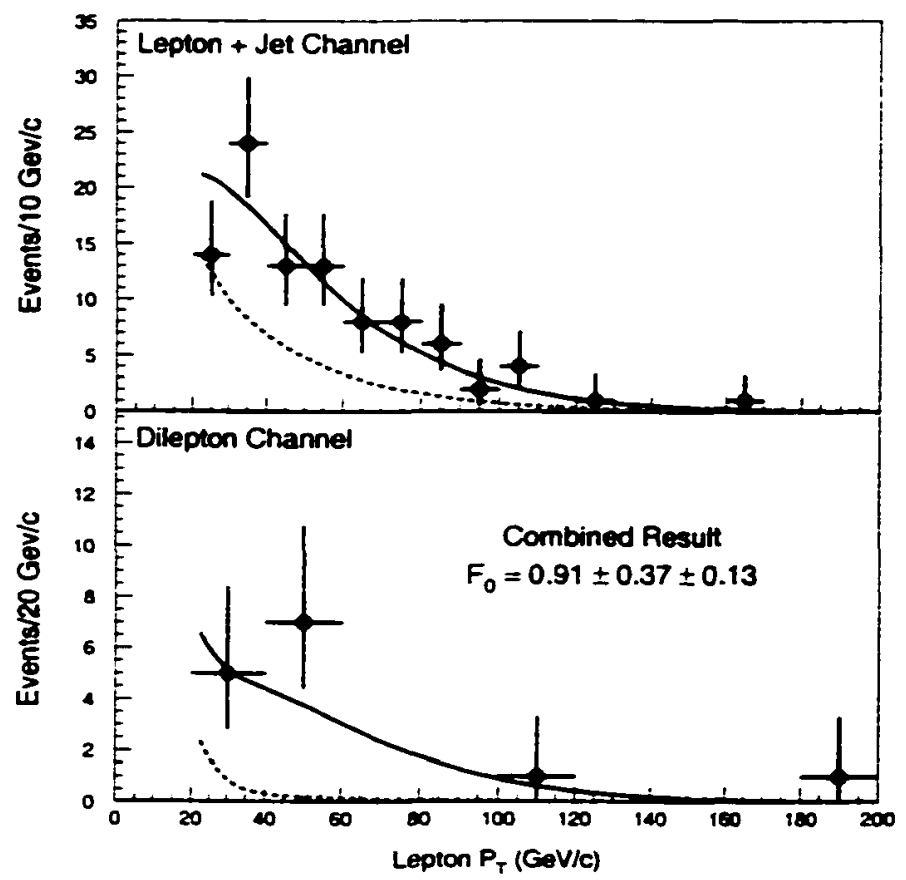

Figure 5.5: Lepton $P_{T}$ distributions for the lepton+jet and dilepton subsamples. The lepton+jet subsamples are added together to simplify presentation. The data (points) are compared with the result of the combined fit (solid line) and with the background component of the fit (dashed line).

is driven by Event $67581 / 129896$, which has a positron with an $E_{T}$ of 181 . Recall that fit quality is determined by comparing the size of the log-likelihood that is returned from the fit to the data with the ensemble of log-likelihood values returned by 1000 pseudo-experiments conducted with an identical sample size. A glance at Figure 5.5 will confirm that no fit configuration could fail to return an abnormally high negative log-likelihood with this event present because all templates have very low probability at high $\boldsymbol{P}_{T}$.

\subsection{Measurement of $\mathcal{F}_{+}$}

The techniques described thus far in this chapter can be modified to measure the fraction of positive helicity $W$ bosons in top quark decays, $\mathcal{F}_{+} \cdot$ We test our ability to do 


\begin{tabular}{|l|c|c|c|c|}
\hline Sample & Best Fit & Corrected Result & Expected Sensitivity & Fit Quality \\
\hline SVX tagged & $0.92_{-.41}^{+.41}$ & 0.90 & \pm 0.42 & $22 \%$ \\
SLT tagged & $-0.07_{-.27}^{+.91}$ & & \pm 0.8 & $75 \%$ \\
Notag & $1.15_{-.70}^{+.98}$ & & \pm 0.65 & $39 \%$ \\
\hline dilepton & $0.60_{-.47}^{+.57}$ & 0.56 & \pm 0.59 & $5.6 \%$ \\
\hline Simultaneous Fit & $0.93_{-.32}^{+.32}$ & 0.91 & \pm 0.30 & $31 \%$ \\
\hline
\end{tabular}

Table 5.4: Fits to the data for the longitudinal $W$ fraction using GGWIG templates.

\begin{tabular}{|cccc|}
\hline $\begin{array}{c}\text { VECBOS } \\
\text { only }\end{array}$ & $\begin{array}{c}17.5 \% \text { non-isolated } \\
\text { leptons }\end{array}$ & $\begin{array}{c}22.7 \% \text { non-isolated } \\
\text { leptons }\end{array}$ & $\begin{array}{c}27.6 \% \text { non-isolated } \\
\text { leptons }\end{array}$ \\
\hline $0.00 \pm 0.14$ & $0.10 \pm 0.15$ & $0.11 \pm 0.14$ & $0.13 \pm 0.14$ \\
\hline
\end{tabular}

Table 5.5: The table entries contain the right-handed fraction returned from fits to the data with various mixes of non-isolated leptons in the background model.

a likelihood fit for the positive helicity fraction by using pseudo-experiments. In Figure 5.6 we show a distribution of 1000 pseudo-experiment fits where the input value of $\mathcal{F}_{+}$was 0 . The distribution is indeed centered at zero, and has a width of 0.15 . This width is small compared to the width of 0.30 obtained when performing pseudo-experiments for the longitudinal measurement, but it is large compared to the physical range for the righthanded fraction of 0.0 to 0.25 . The range is restricted because we fix the longitudinal fraction to 0.75 .

Next we apply our likelihood fit to the data. We show in table 5.5 the best fit value returned for several admixtures of non-isolated leptons into the background model. The central value is that which is returned when the background contains $23 \%$ non-isolated leptons, and is $\mathcal{F}_{+}=0.11 \pm 0.15$ (statistical uncertainty only). The other fit values are used to establish the size of the lepton + jet background composition uncertainty, just as in the longitudinal analysis. 
Pseudo-Experiments for Right-Handed W Fraction

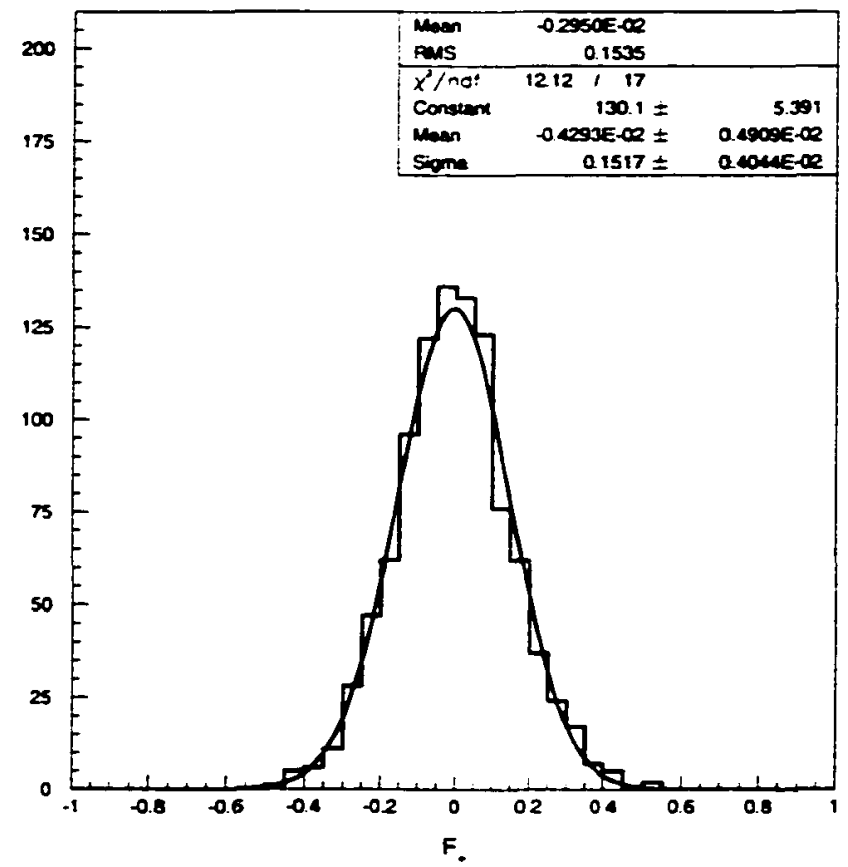

Figure 5.6: Results of a pseudo-experiment study of the sensitivity of the positive helicity measurement. The width of the Gaussian distribution is only 0.15 , but when compared to the width of the physically allowable region $(0.0$ to 0.30$)$ it appears that we have very little sensitivity. 


\section{Chapter 6}

\section{Systematic Uncertainties}

There are two general categories of systematic uncertainties present in this analysis. These are shape uncertainties and acceptance uncertainties. The shape uncertainties include any effects which can change the shape of the lepton $\boldsymbol{P}_{T}$ spectra and thus alter the longitudinal helicity fraction derived from these shapes. For example, a change in the parton distribution functions could change the $\boldsymbol{P}_{T}$ distribution of the top quark and thus change the $\boldsymbol{P}_{T}$ spectra for the leptons as well. The acceptance uncertainties which we are concerned with include any effects which can alter the ratio of the acceptances of events with negative helicity $W$ bosons to the acceptance of events with longitudinal $W$ 's in them $\left(R_{(-10)}\right)$, and thus change our acceptance correction (Equation 5.4), which depends upon this ratio. In general, both types of uncertainty can be caused by a single physies effect, eg. a harder $P_{T}$ spectrum for leptons from negative helicity $W$ bosons will bias the fit by altering the shape of the template used for negative helicity $W$ bosons and it will result in a larger acceptance of events with negative helicity $W$ bosons in them. We must be careful to account for both effects while we are estimating the systematic uncertainties for this analysis. 


\subsection{Top Mass Bias}

The top mass affects the measurement of $\mathcal{F}_{0}$ in some important ways. First, according to the standard model, it is the parameter which determines the degree of the longitudinal polarization of the $W$ boson. This is not a source of systematic uncertainty; in fact, it is the hypothesis which this measurement proposes to test.

The second effect of the top mass on our measurement is to change the lepton $P_{T}$ distribution for leptons which come from longitudinal $W$ bosons. In Figure 6.1 we show a comparison of GGWIG templates which were generated with masses of 140, 175, and 210 $\mathrm{GeV}$. For the negative helicity templates the shape of the $P_{T}$ spectrum does not change as a function of the mass. However, the $P_{T}$ spectrum for the leptons from longitudinal W's is harder for the high mass top quarks and this could certainly cause a shift in the measured value of $\mathcal{F}_{0}$.

To estimate this effect, we use GGWIG to generate Monte Carlo $t \bar{t}$ events with negative helicity or longitudinal $W$ bosons at the following top masses: $m_{t}=165,170,180$, and185. We then use templates constructed from these Monte Carlo samples to measure $\mathcal{F}_{0}$ as a function of template top mass. We show the results of these measurements in Figure 6.2. Note that the measured $\mathcal{F}_{0}$ decreases as the mass used in the template increases. This occurs because the peak of the lepton $P_{T}$ distribution for longitudinal $W$ bosons shifts to higher values as the top mass used to construct the template increases, and this causes the likelihood function to favor the softer negative helicity template.

The line shown in Figure 6.2 is a best fit to the five measurements in the plot, and has the equation $\mathcal{F}_{0}=-0.0152 m_{t}+3.56$. We plug the uncertainty of $\pm 5.1[26]$ on the top mass measurement into this equation to estimate the size of possible biases in the longitudinal measurement. We find $\delta \mathcal{F}_{0}= \pm 0.08$.

\subsubsection{Non-W Background Normalization}

Our second largest systematic uncertainty is related to the normalization on the non- $W$ background to the lepton + jet data samples. The lepton $P_{T}$ spectrum in our non-W 


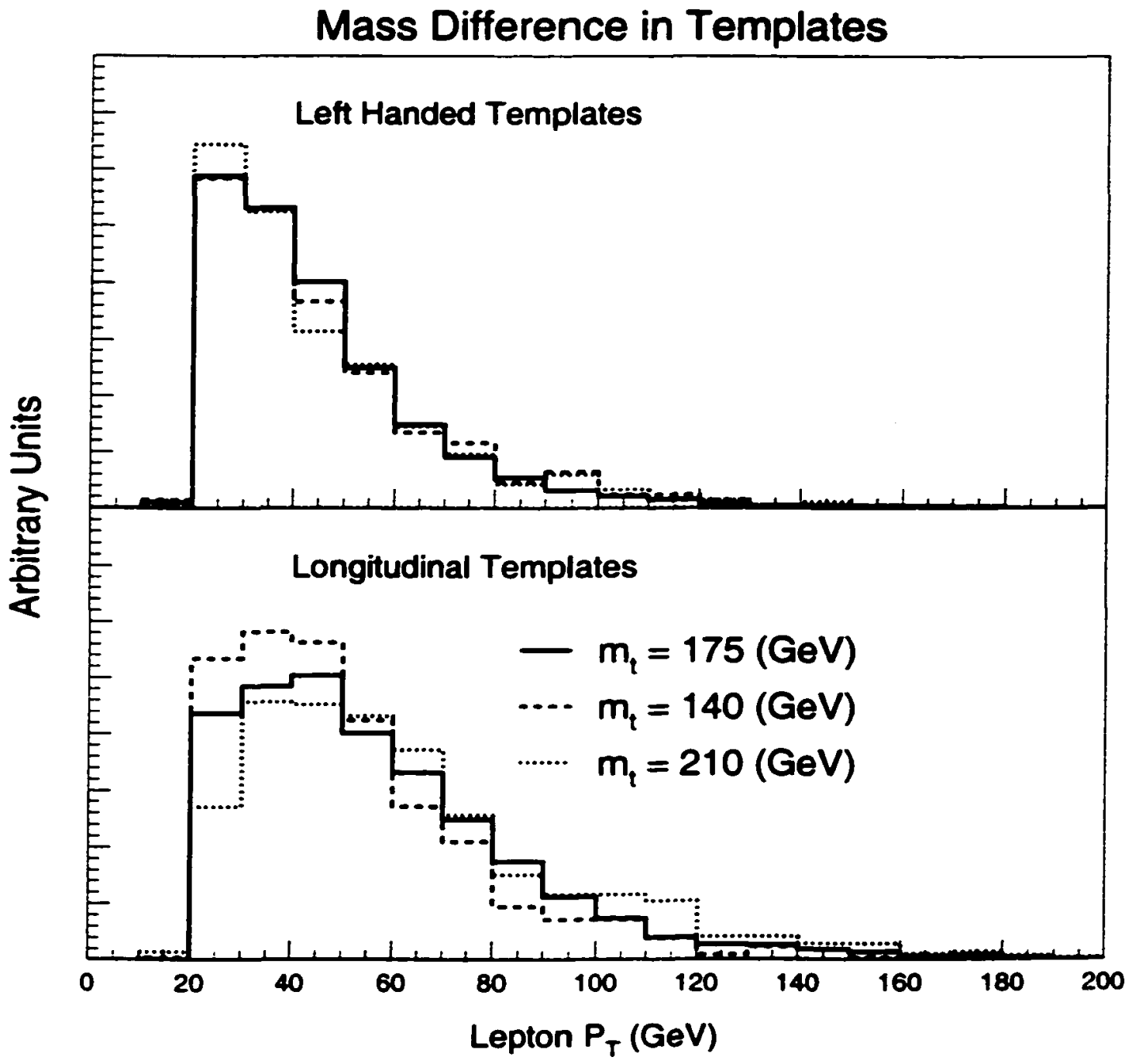

Figure 6.1: Comparison of negative helicity and longitudinal templates at different top mass values. 

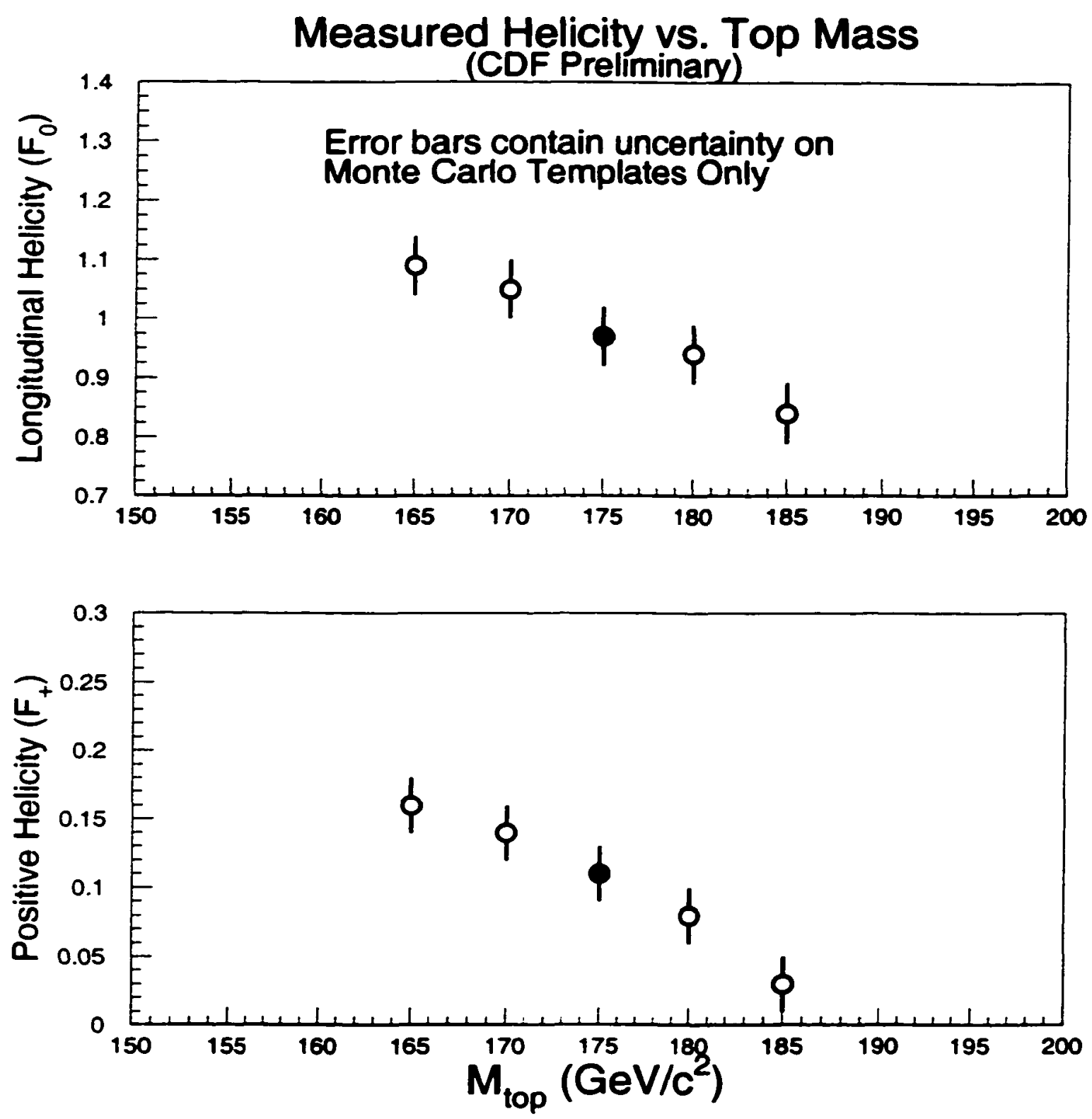

Figure 6.2: Measured helicity fraction versus the mass of top which is used in our analysis templates. The uncertainties include only the uncertainty on the Monte Carlo template because all other uncertainties in these measurements are highly correlated. 
background model shape is similar to the $P_{T}$ spectrum of leptons from negative helicity $W$ bosons in top decay. This means that changes in the amount of non-W background which is included in our background model can exert a strong influence over the value of $\mathcal{F}_{0}$ that is returned by the likelihood minimization. In general, an increase in the non- $W$ content of our background model by $1 \%$ increases $\mathcal{F}_{0}$ by 0.01 . We construct background templates that vary in non- $W$ content by $\pm 4.9 \%$ and then use them to measure $\mathcal{F}_{0}$. We measure the fluctuations in $\mathcal{F}_{0}$ to be $\mathbf{\pm 0 . 0 6}$.

\section{2 b Quark Tagging Efficiency}

Recently some studies at CDF have challenged the $b$ tagging efficiency which is used in this analysis to estimate the size of some of our backgrounds. This change in $b$ tagging efficiency has a large effect on the top cross-section measurement [23] but we will show that its effect on the measurement of $\mathcal{F}_{0}$ is small. For this reason we have decided to list the change in $b$ quark tagging efficiency as a source of systematic uncertainty rather than go to the considerable work of re-calculating all of our backgrounds.

We have examined the effects of using the new $b$ tag efficiencies with a preliminary background calculation, which we summarize in Table 6.1. The most important effect of the new efficiency is the large reduction in the estimate of the non- $W$ contribution to our background. Because this background has a shape which is similar to the lepton $\boldsymbol{P}_{T}$ spectrum from negative helicity $W$ bosons a change in the normalization of the non- $W$ background contribution can change $\mathcal{F}_{0}$. Note that there is no change in the SLT sample. That is because we have no estimate for that sample. We believe that it will change, but since it is a small sample we suspect that any change due to the SLT's will be minor, so we are confident that our estimate of the systematic uncertainty is valid even without an estimate of changes to the SLT sample.

When we measure $\mathcal{F}_{0}$ with these new background calculations we get $\mathcal{F}_{0}=0.88 \pm$ 0.32 , compared to $\mathcal{F}_{0}=0.93 \pm 0.30$ using the old background estimates. Note that we do not apply our acceptance correction these numbers, or to any of the other numbers in 


\begin{tabular}{lccc}
\hline Sample & of events & Old back/Non-W\% & New back/Non-W\% \\
\hline SVX & 34 & $9.2+/-1.2 / 24.1 \%$ & $9.7+/-1.2 / 14.0 \%$ \\
SLT & 14 & $6.0+/-1.2 / 19.0 \%$ & No Change \\
Notag & 46 & $25.9+/-6.5 / 23.1 \%$ & $34.9+/-7.4 / 18.6$ Dileptons \\
7 & $0.76+/-0.21$ & N/A & \\
\hline
\end{tabular}

Table 6.1: Preliminary estimate of changes to the backgrounds when the new $b$ tag efficiencies are applied.

the study of this systematic uncertainty. The acceptance correction was calculated using acceptances for longitudinal and negative helicity W's before tagging, so the change in the tagging efficiency should have no bearing on it, and, in general, the acceptance correction exaggerates any change to the analysis because it is applied as a function of $\mathcal{F}_{0}$.

The change in the central value is mostly due to the change in the non-W background in the SVX channel. The non-W background in the SVX sample has dropped from $24 \%$ of the background to $14 \%$ of the background. This tends to reduce the value of $\mathcal{F}_{0}$, because the non-W background mimics the shape of the negative helicity $W$ bosons.

We summarize the effects of the change in $b$ tagging efficiency by quoting the difference between the measurements of $\mathcal{F}_{0}$ with the old efficiency and the new efficiency as a systematic uncertainty to due to the uncertainty in the $b$ tagging efficiency. This results in a systematic uncertainty of \pm 0.05 in $\mathcal{F}_{0}$.

\subsection{Monte Carlo Statistics}

Our next systematic uncertainty derives from the statistical uncertainty on the shape of our fit templates. We need to estimate how much variation in the shape is possible given the size of our Monte Carlo samples and how large an effect these variations have on our measurement. To estimate the size of this effect we subdivide our Monte Carlo into four orthogonal samples and construct a set of templates from each. We then fit these templates to the data. We take one half of the root mean square of these four measurement as the 
uncertainty due to the statistical uncertainty in the Monte Carlo templates. The result is an uncertainty of \pm 0.05 .

\subsection{Acceptance Uncertainties}

There are a number of uncertainties on the determination of the acceptances which were computed in Section 5.3 and we must determine the degree to which they affect the acceptance correction. Some of these uncertainties are correlated with shape uncertainties (eg. the top mass uncertainty and the gluon radiation uncertainty). We treat these two uncertainties separately so that we can correctly handle the correlations between the acceptance effects and the shape effects. In Table 6.2 we list all acceptance uncertainties which are not correlated with any shape uncertainties. The relative uncertainties in Table 6.2 are extracted from [22] for dilepton acceptances and from [40] for lepton + jet acceptances, we assume that the uncertainties on the helicity dependent acceptances calculated in Section $\mathbf{5 . 3}$ will be identical to those in Table 6.2.

We could, in principle, propagate the uncertainties in Table 6.2 through Eqn. 5.4 and Eqn. 5.7, using standard error propagation techniques (see [41]) and thus obtain the uncertainty on the acceptance correction due to uncertainties on the acceptances. This would be both tedious and difficult. We adopt an alternative approach. We add the relative uncertainties in Table 6.2 in quadrature and then assume that it is appropriate to apply them to the helicity dependent acceptances which were calculated in Section 5.3.1 and 5.3.2. We randomly vary each of the acceptances according to its uncertainty to create 1000 acceptance corrections. We then apply each acceptance correction to the helicity fraction returned by the GGWIG fit to the data to determine how much variation is possible in the size of the acceptance correction. Our acceptance corrected fit values range from 0.89 to 0.93 and have a width of 0.005 .

If the uncertainties of the five helicity dependent acceptances were uncorrelated then it would be appropriate to quote the width of the corrected values as the uncertainty due to the acceptance correction. In general, the uncertainties are correlated, because the 
uncertainties in Table 6.2 are common to all helicity dependent acceptances. We thus quote the range of corrected values $( \pm 0.02)$ as the uncertainty due to acceptance effects.

This is a conservative estimate, because it assumes that there are no correlations in the uncertainties of the five acceptances In fact, there probably are correlations between the acceptances and they will likely reduce the uncertainty on the acceptance correction rather than enhance it, because the acceptances appear in the acceptance correction equations in ratios which will cancel some or all of the uncertainties in Table 6.2.

\begin{tabular}{|l|c|}
\hline Source & Relative Uncertainty \\
\hline \hline Dileptons & \\
\hline Lepton ID efficiencies & $\mathbf{7 \%}$ \\
Jet Energy Scale & $2 \%$ \\
Conversion Removal & $2 \%$ \\
Isolation Efficiency & $2 \%$ \\
Tracking Eficiency & $2 \%$ \\
Trigger Efficiency & $1 \%$ \\
MC statistics & $3 \%$ \\
\hline Lepton + Jet & \\
\hline Jet Energy Scale & $5 \%$ \\
MC Statistics & $3 \%$ \\
\hline
\end{tabular}

Table 6.2: Some Relative Uncertainties on Dilepton and Lepton + Jet acceptances. Only acceptance uncertainties which are uncorrelated to any shape uncertainties are listed in this table. These uncertainties are taken from Ref. [40] and Ref. [22].

\subsection{Non-W Background Shape}

It is important to determine the accuracy of our non-W background model. In particular, we must establish whether or not the non-isolated leptons which we use have the same $P_{T}$ spectrum as the isolated leptons which we are trying to model. If lepton isolation has a strong effect on the lepton $\boldsymbol{P}_{T}$ distribution then the background model based on non-isolated leptons which we constructed in Section 4.2.1 is inaccurate. To address this issue we have generated a sample of $b \bar{b}$ using the PYTHIA Monte Carlo generator. The 
minimum $P_{T}$ of the generated $b \bar{b}$ pair is set to 40 . We also require there to be a lepton with $P_{T}$ greater than 18 at the generator level. We then simulate this $b \bar{b}$ Monte Carlo using QFL' . Finally, we select simulated events by requiring that they have a lepton with $P_{T}$ greater than 20 and three fiducial jets with $E_{T}$ greater than 15 .

In figure 6.3 we show the $P_{T}$ spectra for the $b \bar{b}$ Monte Carlo after dividing the sample into four isolation vs. $F_{T}$ regions:

- region $A: F_{T} \leq 15$ and $I \leq 0.1$

- region $\mathrm{B}: \mathbb{F}_{T} \leq 15$ and $I \geq 0.2$

- region $\mathrm{C}: W_{T} \geq 20$ and $I \geq 0.2$

- region $\mathrm{D}: F_{T} \geq 20$ and $I \leq 0.1$ (W signal region).

Note that the statistics in region $D$, which corresponds to the top signal region, are rather poor. This is due to the difficulty of generating $b \bar{b}$ Monte Carlo which passes our jet cuts, the lepton isolation requirement, and the $E_{T}$ cut. However, we do have many events in the other three regions of isolation vs. FT space, and we will show below that this is enough for our purposes. Note that in figure 6.3 the two plots with isolated leptons do have harder $P_{T}$ spectra than the non-isolated lepton plots. The effect is small, and all four plots have the sharp peak in the 20-30 portion of the spectrum which we believe is responsible for the suppression of the negative helicity component in our fit.

To demonstrate the validity of our $b \bar{b}$ Monte Carlo we compare it to Run I data in a few kinematic variables. The data samples used for these comparisons are regions $B$ and $C$ in the lepton+jets sample, which we expect to be high in $b \bar{b}$ content. We compare these samples to the corresponding regions in our $b \bar{b}$ Monte Carlo. In figure 6.4 we compare lepton $P_{T}$, leading jet $E_{T}, E_{T}$, and event sum $E_{T}$ between data and Monte Carlo in region B. The agreement between data and Monte Carlo in the lepton $P_{T}$ distribution is quite good, but in the other three distributions the Monte Carlo does not model the data very well. The transverse energy distribution of the leading jet is slightly harder in the 
BBbar MC Lepton $\mathrm{P}_{\mathrm{T}}$ Distributions
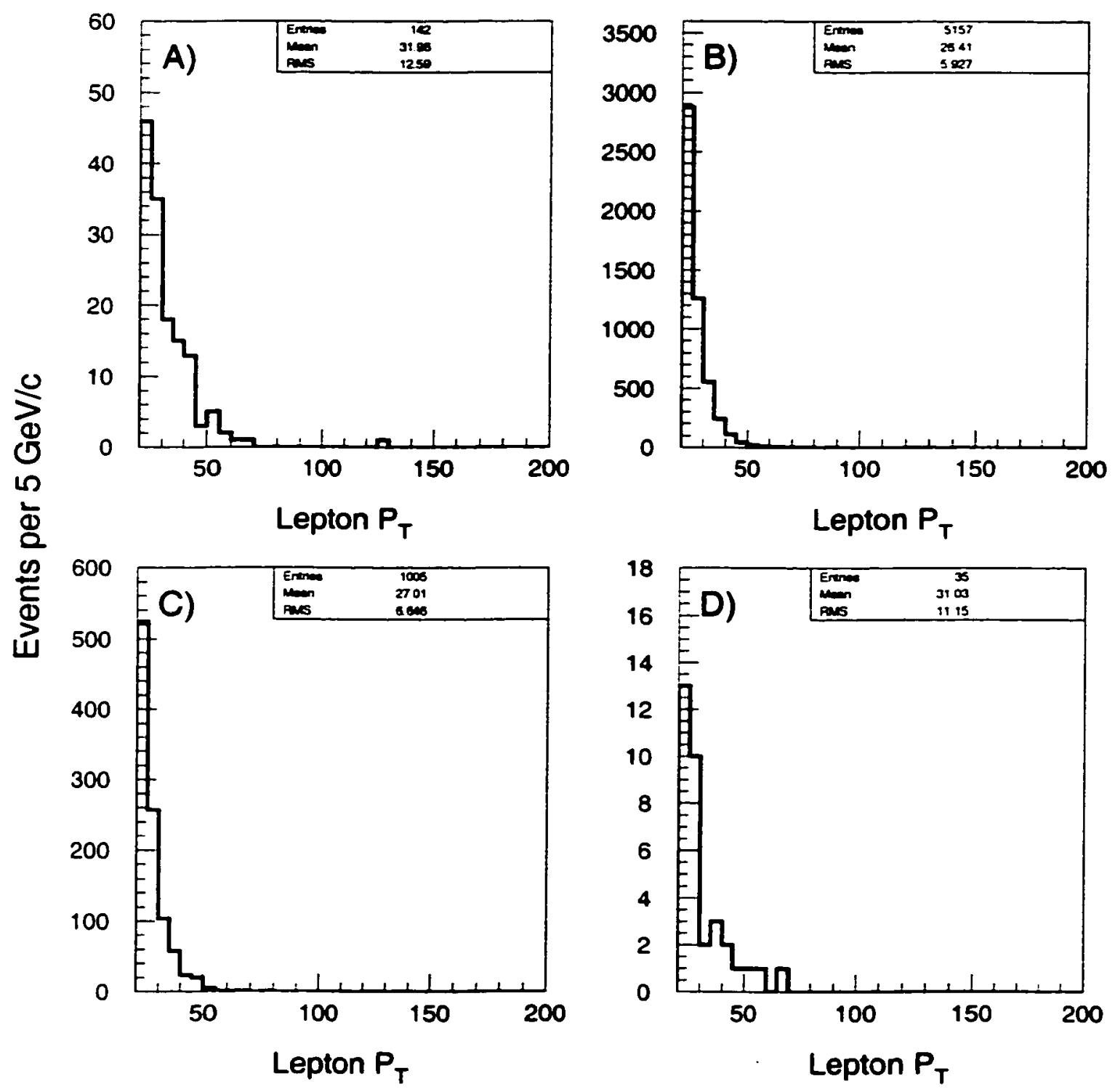

Figure 6.3: Lepton $P_{T}$ spectrum in our PYTHIA b $\bar{b}$ sample as a function of $\psi_{T}$ and lepton isolation. In region $A W_{T}<15$ and $I<0.1$, in region $B \psi_{T}<15$ and $I>0.2$, in region $\mathrm{C}$ $E_{T}>20$ and $I>0.2$, and finally, in region $D H_{T}>20$ and $I<0.1$. 


\begin{tabular}{|lcc|}
\hline Non-W model & Cuts & $\mathcal{F}_{0}$ \\
\hline \hline Vecbos W + jets & $\mathbb{F}_{T} \geq 20, I \leq 0.1$ & 0.57 \\
Run Ib Data & $\mathbb{F}_{T} \geq 20, I \geq 0.2$ & 0.97 \\
Pythia BB MC & $\mathbb{F}_{T} \geq 20, I \leq 0.1$ & 0.94 \\
Pythia BB MC & $\mathbb{F}_{T} \geq 20, I \leq 0.15$ & 0.92 \\
Pythia BB MC & $\mathbb{F}_{T} \geq 15, I \leq 0.1$ & 0.92 \\
Pythia BB MC & $\mathbb{F}_{T} \geq 15, I \leq 0.15$ & 0.92 \\
Pythia BB MC & $\mathbb{F}_{T} \leq 15, I \leq 0.1$ & 0.94 \\
Pythia BB MC & $\mathbb{F}_{T} \geq 20, I \geq 0.2$ & 0.99 \\
\hline
\end{tabular}

Table 6.3: Fit results for the longitudinal fraction using several different non-W background models. One-half the range of the results which use Pythia Monte Carlo as a non-W background model is quoted as the non-W background shape uncertainty.

$b \bar{b}$ Monte Carlo than in the data, and the $E_{T}$ distribution in the Monte Carlo is slightly softer than it is in the data. These disagreements indicate that there may be a significant non- $b \bar{b}$ component to the region $B$ data. However, we are encouraged by the agreement

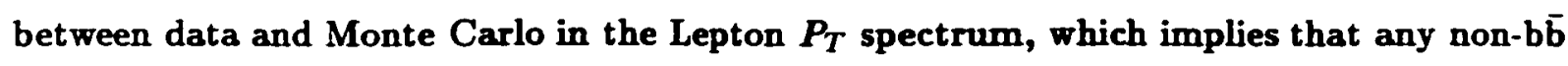
component to the data in region $B$ has a lepton $P_{T}$ spectrum which is similar to $b \bar{b}$.

These same comparisons are made in region $C$ in figure 6.5. In region $C$ the agreement between the $b \bar{b}$ Monte Carlo and the data is reasonable in the lepton $P_{T}$, sum $E_{T}$, and $F_{T}$ distributions. The agreement in the leading jet $E_{T}$ distribution is still poor. Note that region $C$ probably contains some $t \bar{t}$ events, which would help to explain why the leading jet $E_{T}$ distribution is harder in the data than in the Monte Carlo. We claim that the agreement between data and Monte Carlo in the lepton $\boldsymbol{P}_{T}$ distributions in both region $B$ and $C$ is sufficient justification for using this Monte Carlo in our non-W background shape study.

We next fit for the longitudinal $W$ fraction using the $35 \mathrm{~b} \bar{b}$ Monte Carlo events in region $D$ as our non- $W$ background model. The result is $\mathcal{F}_{0}=0.94$, which is very close to the result of $\mathcal{F}_{0}=0.97$ which is obtained when we use run Ib non-isolated lepton data as our non-W background. As a cross-check on this result, we also perform the fit using Monte Carlo data in region $A$, which has low $W_{T}$ and isolated leptons; and region $C$, which 


\section{Kinematic Comparisons Between Pythia and Data (Region B)}
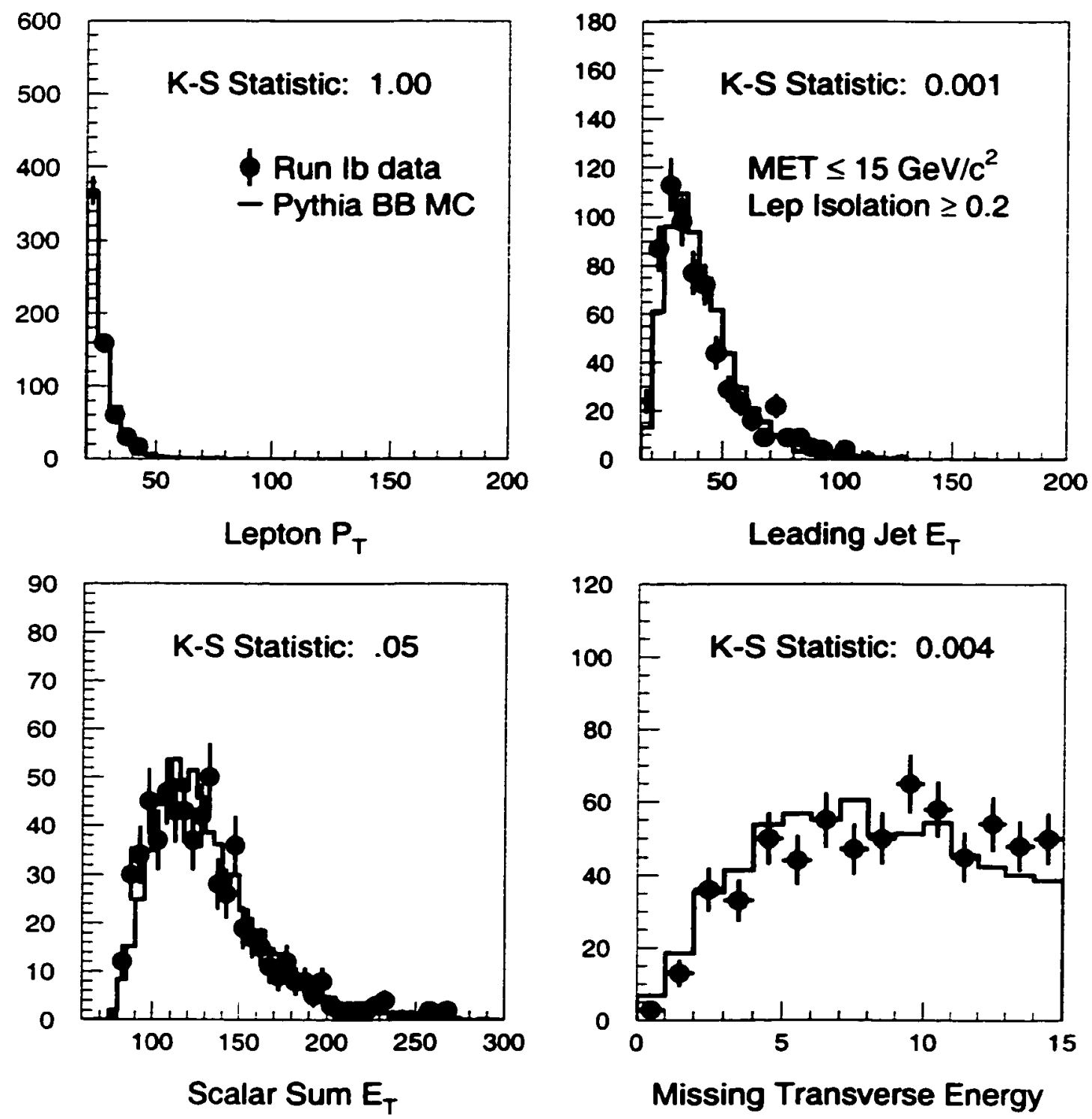

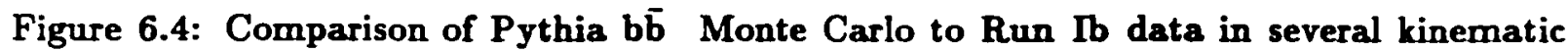
variables. The events in these plots come from region $B$. 


\section{Kinematic Comparisons Between Pythia and Data (Region C)}
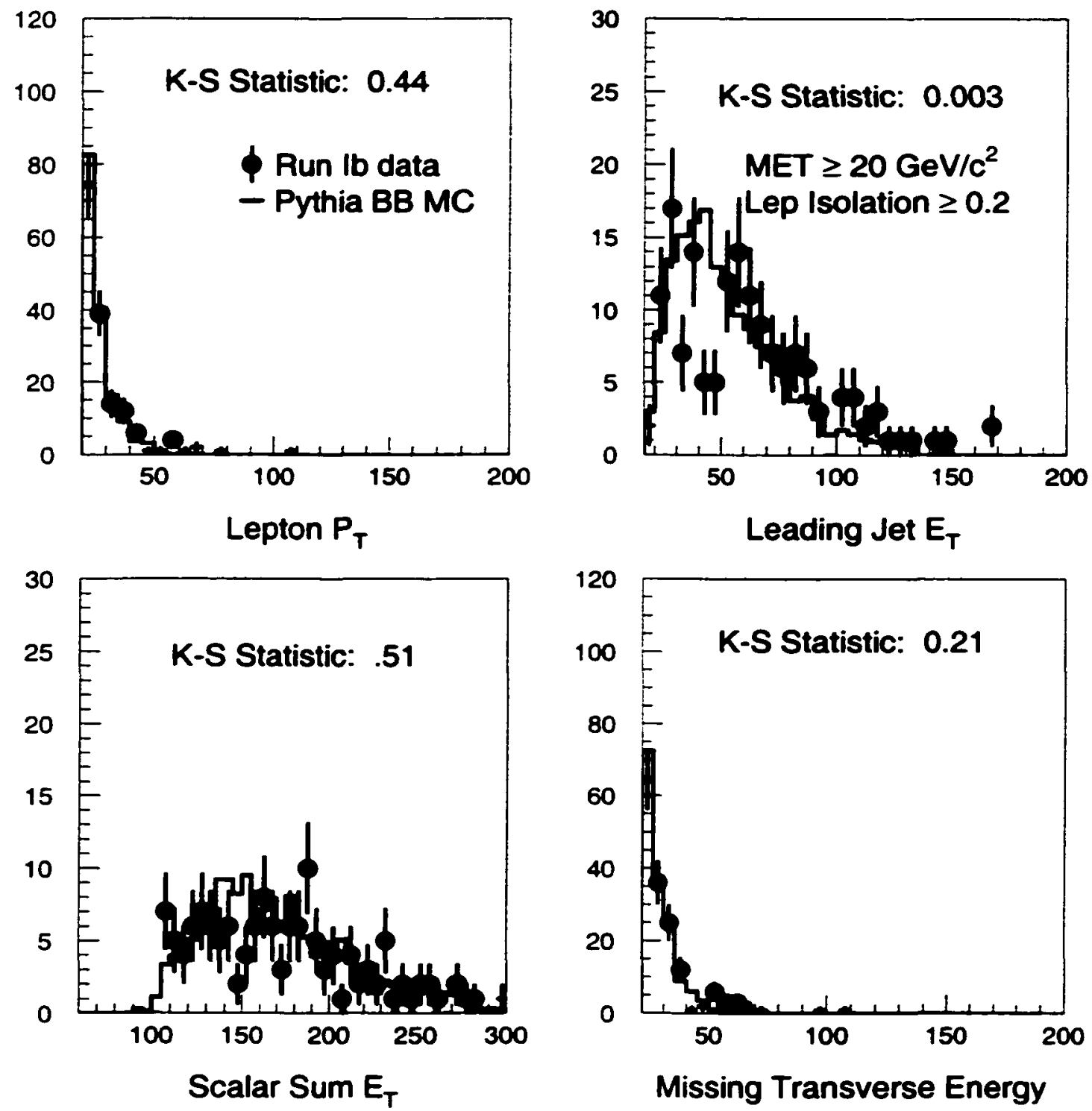

Figure 6.5: Comparison of Pythia $b \bar{b}$ Monte Carlo to Run $\mathrm{lb}$ data in several kinematic variables. The events in these plots come from region $C$. 
has large $E_{T}$ and non-isolated leptons. We also loosen the isolation and $\mathbb{F}_{T}$ cuts in region $D$ of the Monte Carlo in order to increase the statistics in the most top-like Monte Carlo sample. The results of fits performed with these alternative non- $W$ background models are summarized in Table 6.3. These fit results range between 0.92 and 0.99 . We take half this range to be the systematic uncertainty on the shape of the non-W background.

\subsection{Gluon Radiation}

Gluon radiation is a traditional source of uncertainty in most top analyses, and clearly has a direct impact on the $\boldsymbol{P}_{T}$ of the top and its decay products. We estimate the effects of gluon radiation on our measurement by turning on and off the initial and final state radiation in Pythia MC. We show the effect that turning off initial or final state radiation has on the lepton $P_{T}$ spectrum in Figure 6.6. Turning off the initial state radiation has very little effect on the lepton $\boldsymbol{P}_{T}$ spectrum in the lepton + jets sample. A K-S test between Pythia with ISR and Pythia without it demonstrates that the distributions are the same at the $\mathbf{9 8 \%}$ confidence level. Turning off the final state radiation does affect the lepton $\boldsymbol{P}_{T}$ spectrum. The sample without FSR has a slightly harder $P_{T}$ spectrum. A K-S test between these two shapes results in 0.09 .

In the dilepton Pythia samples, the K-S statistic between the NO-ISR sample and the sample with all radiation on is 0.99 and the $\mathrm{K}$-S statistic between the NO-FSR and all rad on samples is 1.00 .

It is also possible that the radiation model used in our Monte Carlo model could affect our acceptance correction. In Section 5.3.3 we discuss a technique for measuring helicity dependent acceptances with Pythia and then list in Table 5.3 at the end of that section the results of this study. We found that the effect of gluon radiation upon our acceptance correction was minimal, because the change in acceptance was insignificant in the dilepton sample, and in the lepton + jet sample the small change in acceptance canceled in the ratio $R_{(-/ 0)}$.

Armed with the knowledge that the change in lepton $P_{T}$ spectrum and acceptance 


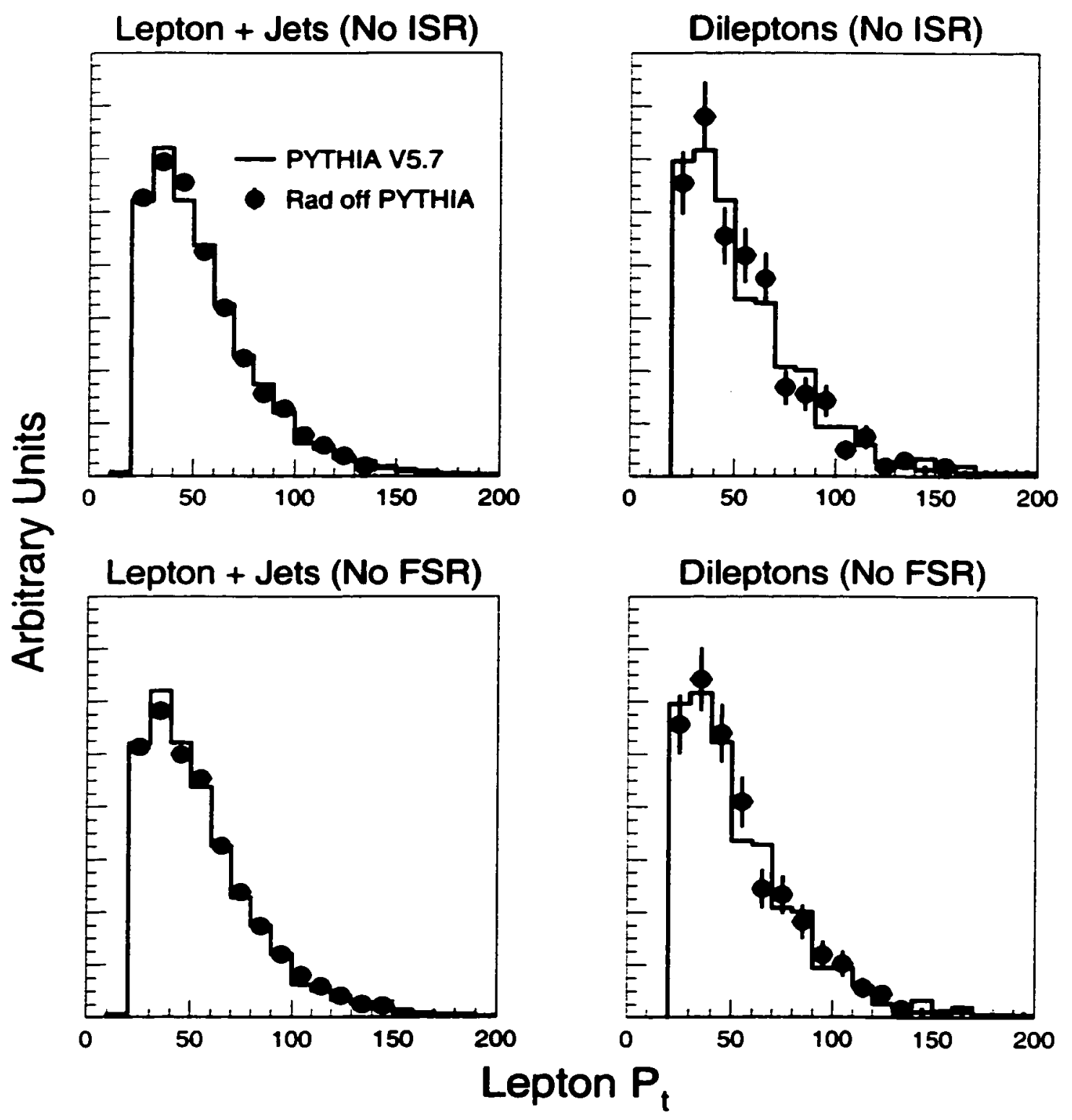

Figure 6.6: Comparison of the Pythia lepton $\boldsymbol{P}_{T}$ spectrum with radiation on to the Pythia lepton $P_{T}$ spectrum with initial or final state radiation turned off. 
is small, we can predict that gluon radiation has little influence upon the measurement of the longitudinal helicity fraction. To obtain a quantitative measure of the size of the effect of gluon radiation, we use pseudo-experiments. First, we select the input helicity fraction to our pseudo-experiments by using Eqn. 5.3 and Eqn. 5.6 with the acceptance parameters listed in Table 5.3 to predict the size of the acceptance bias for $t \vec{t}$ production in which initial state radiation or final state radiation has been turned off. We use templates constructed from Pythia samples with radiation turned off to produce the Pseudo-events, and then measure the helicity fraction using templates constructed from Pythia with all radiation turned on. Finally, we correct the mean of the returned $\mathcal{F}_{0}^{\text {fit }}$ values with acceptance parameters derived from radiation on Pythia (also listed in Table 5.3). This procedure accounts for both shape effects and acceptance effects. We find that turning the initial state radiation off causes us to underestimate the true helicity fraction by 0.02 , and turning final state radiation off causes us to overestimate it by 0.02 . Adding these uncertainties in quadrature, we obtain $\pm \mathbf{0 . 0 3}$. We quote this as the systematic uncertainty from our model of gluon radiation.

\subsection{Parton Distribution Functions}

We can estimate the uncertainty in our fit due to the uncertainty in the parton distribution functions used to generate our templates by generating new templates using

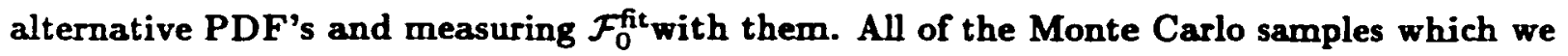
have discussed so far were generated using the MRSD0' parton distribution functions. We also have a sample of over 8000 Herwig t $\bar{t}$ events generated using the CTEQ4L PDF's. We measure $\mathcal{F}_{0}^{\text {fit }}$ for this sample by dumping all 8000 lepton $P_{T}$ values into a file and then fit them with our GGWIG templates. The result is $\mathcal{F}_{0}^{\text {fit }}=0.796 \pm 0.022$. We compare this to another sample of 10000 Herwig events that were generated with the MRSD0' PDF's. Dumping the lepton $P_{T}$ values from this second sample into a file and fitting them with our GGWIG templates give $\mathcal{F}_{0}^{\text {fit }}=0.778 \pm 0.020$. We quote the difference between these two values of 0.02 as our PDF uncertainty. 


\subsection{Total Systematic Uncertainty}

We find that there are eight primary sources of systematic uncertainty in the measurement of the longitudinal fraction of $W$ bosons in top decay, and we list them in Table 6.4. The total systematic uncertainty is \pm 0.13 , this is negligible compared to the statistical uncertainty of \pm 0.37 . We believe that our estimates of the systematic uncertainty are conservative.

\begin{tabular}{|l|c|}
\hline Source & $\begin{array}{c}\text { Change in } \\
\text { Longitudinal Fraction }\left(\mathcal{F}_{0}\right)\end{array}$ \\
\hline \hline Top Mass Uncertainty & 0.07 \\
Non-W Background Normalization & 0.06 \\
b-tag efficiency & 0.05 \\
Monte Carlo statistics & 0.05 \\
Acceptance Uncertainties & 0.02 \\
Non-W Background Shape & 0.04 \\
Gluon Radiation & 0.03 \\
Structure functions & 0.02 \\
\hline \hline Systematic Uncertainty & 0.13 \\
\hline Statistical Uncertainty & 0.37 \\
\hline Total Uncertainty & 0.39 \\
\hline
\end{tabular}

Table 6.4: Table of uncertainties 


\section{Chapter 7}

\section{Conclusions}

We have measured the fraction of $W$ bosons that are produced with zero helicity in the decay of top quarks to be $\mathcal{F}_{0}=0.91 \pm 0.37 \pm 0.13$. This is consistent with the standard model prediction of $\mathcal{F}_{0}=0.70$ for a top mass of $175 \mathrm{GeV} / c^{2}$. The small size of our data sample precludes a more precise measurement at this time, but we have demonstrated that the lepton $\boldsymbol{P}_{T}$ spectrum is a simple and effective tool for probing an important dynamical feature of top quark decays. This is the first direct measurement of a property of the top quark other than its mass or cross-section. We have also measured the fraction of $W$ bosons with positive helicity in top quark decays to be $\mathcal{F}_{+}=0.11 \pm 0.15$, assuming $\mathcal{F}_{0}=0.70$. This is consistent with the standard model prediction that $\mathcal{F}_{+}=\mathbf{0 . 0}$. With current statistics it is not possible to place a meaningful upper limit on $\mathcal{F}_{+}$.

It is important to measure the helicity of $W$ bosons in top quark decays for a number of reasons. The large mass of the top quark implies that its coupling to the Higgs boson is large, and this in turn hints that the top quark may play a special role in the origin of mass. We may find clues to this special role in the nature of the top quark's coupling to the $W$ boson, which is dominated by the Goldstone mode of the Higgs doublet.

The measurement of the helicity of the $W$ can also provide further confirmation that the particle which was discovered by the CDF and DO collaborations $[2,3]$ is indeed the top quark. The chiral nature of the coupling of the top quark to the $W$ boson imposes a structure upon the $P_{T}$ distribution of electrons and muons into which the $W$ decays. 
We have parametrized this structure in the lepton $\boldsymbol{P}_{T}$ distribution in terms of the helicity of the $W$, as predicted by the standard model. If some or all of the particles in the top data sample were new particles which mimic the decay signature of the top quark then this parameterization would not be valid, and the measurement might deviate substantially from the prediction of $\mathcal{F}_{0}=\mathbf{0 . 7 0}$.

This measurement can also be used to improve the tools which are used in other top quark kinematic analyses. The CDF top mass measurement [26] uses a kinematic fitter which determines the mass, momentum, and parentage of each particle in the lepton + jets data sample via a log-likelihood fit to the $t \bar{t}$ hypothesis. This kinematic fitter can be used to measure the helicity of the $W$ directly from the decay angle of the lepton. The accuracy of the fitter is limited by a poor resolution on the momentum of the neutrino and a difficulty with the combinatoric problem of determining which bottom quark goes with which top quark. It may be possible to improve the kinematic fitter by comparing the measurement of the $W$ helicity from the lepton $P_{T}$ spectrum to the measurement from the lepton decay angle and then investigating any discrepancy between the two.

It will be possible to increase the precision of this measurement substantially in the next collider run at the Tevatron. According to predictions from [42], with $2 \mathrm{fb}^{-1}$ of data CDF will collect 1350 lepton +jet events, with a background of 350 events, and 120 dilepton events, with a background of 10 events. Using the pseudo-experiement technique outlined in Section 5.2.1, we predict a statistical uncertainty of \pm 0.07 on $\mathcal{F}_{0}$, and an uncertainty of \pm 0.03 on $\mathcal{F}_{+}$. To improve the precision of the measurement any further it will be necessary to reduce the size of the systematic biases that were estimated in this thesis.

In conclusion, we have used the CDF detector apparatus to measure the helicity of the $W$ boson in top quark decays. The measurement is statistically limited, but we find that the result is consistent with standard model predictions. This is the first measurement of a dynamical feature of the top quark. The precision of the measurement can be increased dramatically in the next collider run at the Tevatron. 


\section{Appendices}




\section{Appendix A}

\section{The CDF Collaboration}

T. Affolder, ${ }^{21}$ H. Akimoto, ${ }^{42}$ A. Akopian, ${ }^{35}$ M. G. Albrow, ${ }^{10}$ P. Amaral, ${ }^{7}$ S. R. Amendolia, ${ }^{31}$

D. Amidei, ${ }^{24}$ J. Antos, ${ }^{1}$ G. Apollinari, ${ }^{35}$ T. Arisawa, ${ }^{42}$ T. Asakawa, ${ }^{40}$ W. Ashmanskas, ${ }^{7}$ M. Atac, ${ }^{10}$ P. Azzi-Bacchetta, ${ }^{29}$ N. Bacchetta, ${ }^{29}$ M. W. Bailey, ${ }^{26}$ S. Bailey, ${ }^{14}$ P. de Barbaro, ${ }^{34}$ A. Barbaro-Galtieri, ${ }^{21}$ V. E. Barnes, ${ }^{33}$ B. A. Barnett, ${ }^{17}$ M. Barone, ${ }^{12}$ G. Bauer, ${ }^{22}$ F. Bedeschi, ${ }^{31}$ S. Belforte, ${ }^{39}$ G. Bellettini, ${ }^{31}$ J. Bellinger, ${ }^{43}$ D. Benjamin, ${ }^{9}$ J. Bensinger, ${ }^{4}$ A. Beretvas, ${ }^{10}$ J. P. Berge, ${ }^{10}$ J. Berryhill, ${ }^{7}$ S. Bertolucci, ${ }^{12}$ B. Bevensee, ${ }^{30}$ A. Bhatti, ${ }^{35}$ C. Bigongiari, ${ }^{31}$ M. Binkley, ${ }^{10}$ D. Bisello, ${ }^{29}$ R. E. Blair, ${ }^{2}$ C. Blocker, ${ }^{4}$ K. Bloom, ${ }^{24}$ B. Blumenfeld, ${ }^{17}$ B. S. Blusk, ${ }^{34}$ A. Bocci, ${ }^{31}$ A. Bodek, ${ }^{34}$ W. Bokhari, ${ }^{30}$ G. Bolla, ${ }^{33}$ Y. Bonushkin, ${ }^{5}$ D. Bortoletto, ${ }^{33}$ J. Boudreau, ${ }^{32}$ A. Brandl, ${ }^{26}$ S. van den Brink, ${ }^{17}$ C. Bromberg, ${ }^{25}$ N. Bruner, ${ }^{26}$ E. Buckley-Geer, ${ }^{10}$ J. Budagov, ${ }^{8}$ H. S. Budd, ${ }^{34}$ K. Burkett, ${ }^{14}$ G. Busetto, ${ }^{29}$ A. Byon-Wagner, ${ }^{10}$ K. L. Byrum, ${ }^{2}$ M. Campbell, ${ }^{24}$ A. Caner, ${ }^{31}$ W. Carithers, ${ }^{21}$ J. Carlson, ${ }^{24}$ D. Carlsmith, ${ }^{43}$ J. Cassada, ${ }^{34}$ A. Castro, ${ }^{29}$ D. Cauz, ${ }^{39}$ A. Cerri, ${ }^{31}$ P. S. Chang, ${ }^{1}$ P. T. Chang, ${ }^{1}$ J. Chapman, ${ }^{24}$ C. Chen, ${ }^{30}$ Y. C. Chen, ${ }^{1}$ M. -T. Cheng, ${ }^{1}$ M. Chertok, ${ }^{37}$ G. Chiarelli, ${ }^{31}$ I. Chirikov-Zorin, ${ }^{8}$ G. Chlachidze, ${ }^{8}$ F. Chlebana, ${ }^{10}$ L. Christofek, ${ }^{16}$ M. L. Chu, ${ }^{1}$ S. Cihangir, ${ }^{10}$ C. I. Ciobanu, ${ }^{27}$ A. G. Clark, ${ }^{13}$ M. Cobal, ${ }^{31}$ E. Cocca, ${ }^{31}$ A. Connolly, ${ }^{21}$ J. Conway, ${ }^{36}$ J. Cooper, ${ }^{10}$ M. Cordelli, ${ }^{12}$ J. Guimaraes da Costa, ${ }^{24}$ D. Costanzo, ${ }^{31}$ J. Cranshaw, ${ }^{38}$ D. Cronin-Hennessy, ${ }^{9}$ R. Cropp, ${ }^{23}$
R. Culbertson, ${ }^{7}$
D. Dagenhart, ${ }^{41}$
F. DeJongh, 10
S. Dell'Agnello, ${ }^{12}$
M. Dell'Orso, ${ }^{31}$ 
R. Demina, ${ }^{10}$ L. Demortier, ${ }^{35}$ M. Deninno, ${ }^{3}$ P. F. Derwent, ${ }^{10}$ T. Devlin, ${ }^{36}$ J. R. Dittmann, ${ }^{10}$ S. Donati, ${ }^{31}$ J. Done, ${ }^{37}$ T. Dorigo, ${ }^{14}$ N. Eddy, ${ }^{16}$ K. Einsweiler, ${ }^{21}$ J. E. Elias, ${ }^{10}$ E. Engels, Jr., ${ }^{32}$ W. Erdmann, ${ }^{10}$ D. Errede, ${ }^{16}$ S. Errede, ${ }^{16}$ Q. Fan, ${ }^{34}$ R. G. Feild, ${ }^{44}$ C. Ferretti, ${ }^{31}$ I. Fiori, ${ }^{3}$ B. Flaugher, ${ }^{10}$ G. W. Foster, ${ }^{10}$ M. Franklin, ${ }^{14}$ J. Freeman, ${ }^{10}$ J. Friedman, ${ }^{22}$ Y. Fukui, ${ }^{20}$ S. Gadomski, ${ }^{23}$ S. Galeotti, ${ }^{31}$ M. Gallinaro, ${ }^{35}$ T. Gao, ${ }^{30}$ M. Garcia-Sciveres, ${ }^{21}$ A. F. Garfinkel, ${ }^{33}$ P. Gatti, ${ }^{29}$ C. Gay, ${ }^{44}$ S. Geer, ${ }^{10}$ D. W. Gerdes, ${ }^{24}$ P. Giannetti, ${ }^{31}$ P. Giromini, ${ }^{12}$ V. Glagolev, ${ }^{8}$ M. Gold, ${ }^{26}$ J. Goldstein, ${ }^{10}$ A. Gordon, ${ }^{14}$ A. T. Goshaw, ${ }^{9}$ Y. Gotra, ${ }^{32}$ K. Goulianos, ${ }^{35}$ H. Grassmann, ${ }^{39}$ C. Green, ${ }^{33}$ L. Groer, ${ }^{36}$ C. Grosso-Pilcher, ${ }^{7}$ M. Guenther, ${ }^{33}$ G. Guillian, ${ }^{24}$ R. S. Guo, ${ }^{1}$ C. Haber, ${ }^{21}$ E. Hafen, ${ }^{22}$ S. R. Hahn, ${ }^{10}$ C. Hall, ${ }^{14}$ T. Handa, ${ }^{15}$ R. Handler, ${ }^{43}$ W. Hao, ${ }^{38}$ F. Happacher, ${ }^{12}$ K. Hara, ${ }^{40}$ A. D. Hardman, ${ }^{33}$ R. M. Harris, ${ }^{10}$ F. Hartmann, ${ }^{18}$ K. Hatakeyama, ${ }^{35}$ J. Hauser, ${ }^{5}$ J. Heinrich, ${ }^{30}$ A. Heiss, ${ }^{18}$ B. Hinrichsen, ${ }^{23}$ K. D. Hoffman, ${ }^{33}$ C. Holck, ${ }^{30}$ R. Hollebeek, ${ }^{30}$ L. Holloway, ${ }^{16}$ R. Hughes, ${ }^{27}$ J. Huston, ${ }^{25}$ J. Huth, ${ }^{14}$ H. Ikeda, ${ }^{40}$ M. Incagli, ${ }^{31}$ J. Incandela, ${ }^{10}$ G. Introzzi, 31 J. Iwai, ${ }^{42}$ Y. Iwata, ${ }^{15}$ E. James, ${ }^{24}$ H. Jensen, ${ }^{10}$ M. Jones, ${ }^{30}$ U. Joshi, ${ }^{10}$ H. Kambara, ${ }^{13}$ T. Kamon, ${ }^{37}$ T. Kaneko, ${ }^{40}$ K. Karr, ${ }^{41}$ H. Kasha, ${ }^{44}$ Y. Kato, ${ }^{28}$ T. A. Keaffaber, ${ }^{33}$ K. Kelley, ${ }^{22}$ M. Kelly, ${ }^{24}$ R. D. Kennedy, ${ }^{10}$ R. Kephart, ${ }^{10}$ D. Khazins, ${ }^{9}$ T. Kikuchi, ${ }^{40}$ M. Kirk, ${ }^{4}$ B. J. Kim, ${ }^{19}$ H. S. $\mathrm{Kim}^{23}$ S. H. Kim, ${ }^{40}$ Y. K. Kim, ${ }^{21}$ L. Kirsch, ${ }^{4}$ S. Klimenko, ${ }^{11}$ D. Knoblauch, ${ }^{18}$ P. Koehn, ${ }^{27}$ A. Köngeter, ${ }^{18}$ K. Kondo, ${ }^{42}$ J. Konigsberg, ${ }^{11}$ K. Kordas, ${ }^{23}$ A. Korytov, ${ }^{11}$ E. Kovacs, ${ }^{2}$ J. Kroll, ${ }^{30}$ M. Kruse, ${ }^{34}$ S. E. Kuhlmann, ${ }^{2}$ K. Kurino, ${ }^{15}$ T. Kuwabara, ${ }^{40}$ A. T. Laasanen, ${ }^{33}$ N. Lai, $^{7}$ S. Lami, ${ }^{35}$ S. Lammel, ${ }^{10}$ J. I. Lamoureux, ${ }^{4}$ M. Lancaster, ${ }^{21}$ G. Latino, ${ }^{31}$ T. LeCompte, ${ }^{2}$ A. M. Lee IV, ${ }^{9}$ S. Leone, ${ }^{31}$ J. D. Lewis, ${ }^{10}$ M. Lindgren, ${ }^{5}$ T. M. Liss, ${ }^{16}$ J. B. Liu, ${ }^{34}$ Y. C. Liu, ${ }^{1}$ N. Lockyer, ${ }^{30}$ M. Loreti, ${ }^{29}$ D. Lucchesi, ${ }^{29}$ P. Lukens, ${ }^{10}$ S. Lusin, ${ }^{43}$ J. Lys, ${ }^{21}$ R. Madrak, $^{14}$ K. Maeshima, ${ }^{10}$ P. Maksimovic, ${ }^{14}$ L. Malferrari, ${ }^{3}$ M. Mangano, ${ }^{31}$ M. Mariotti, ${ }^{29}$ G. Martignon, ${ }^{29}$ A. Martin, ${ }^{44}$ J. A. J. Matthews, ${ }^{26}$ P. Mazzanti, $^{3}$ K. S. McFarland, ${ }^{34}$ P. McIntyre, ${ }^{37}$ E. McKigney, ${ }^{30}$ M. Menguzzato, ${ }^{29}$ A. Menzione, ${ }^{31}$ E. Meschi, ${ }^{31}$ C. Mesropian, ${ }^{35}$ C. Miao, ${ }^{24}$ T. Miao, ${ }^{10}$ R. Miller, ${ }^{25}$ J. S. Miller, ${ }^{24}$ H. Minato, ${ }^{40}$ S. Miscetti, ${ }^{12}$ M. Mishina, ${ }^{20}$ N. Moggi, ${ }^{31}$ E. Moore, ${ }^{26}$ R. Moore, ${ }^{24}$ Y. Morita, ${ }^{20}$ A. Mukherjee, ${ }^{10}$ T. Muller, ${ }^{18}$ 
A. Munar, ${ }^{31}$ P. Murat, ${ }^{31}$ S. Murgia, ${ }^{25}$ M. Musy, ${ }^{39}$ J. Nachtman, ${ }^{5}$ S. Nahn, ${ }^{44}$ H. Nakada, ${ }^{40}$

T. Nakaya, ${ }^{7}$ I. Nakano, ${ }^{15}$ C. Nelson, ${ }^{10}$ D. Neuberger, ${ }^{18}$ C. Newman-Holmes, ${ }^{10}$ C.Y. P. Ngan, ${ }^{22}$ P. Nicolaidi, ${ }^{39}$ H. Niu, ${ }^{4}$ L. Nodulman, ${ }^{2}$ A. Nomerotski, ${ }^{11}$ S. H. Oh, ${ }^{9}$ T. Ohmoto, ${ }^{15}$ T. Ohsugi, ${ }^{15}$ R. Oishi, ${ }^{40}$ T. Okusawa, ${ }^{28}$ J. Olsen, ${ }^{43}$ C. Pagliarone, ${ }^{31}$ F. Palmonari, ${ }^{31}$ R. Paoletti, ${ }^{31}$ V. Papadimitriou, ${ }^{38}$ S. P. Pappas, ${ }^{44}$ A. Parri, ${ }^{12}$ D. Partos, ${ }^{4}$ J. Patrick, ${ }^{10}$ G. Pauletta, ${ }^{39}$ M. Paulini, ${ }^{21}$ A. Perazzo, ${ }^{31}$ L. Pescara, ${ }^{29}$ T. J. Phillips, ${ }^{9}$ G. Piacentino, ${ }^{31}$ K. T. Pitts, ${ }^{10}$ R. Plunkett, ${ }^{10}$ A. Pompos, ${ }^{33}$ L. Pondrom, ${ }^{43}$ G. Pope, ${ }^{32}$ F. Prokoshin, ${ }^{8}$ J. Proudfoot, ${ }^{2}$ F. Ptohos, ${ }^{12}$ G. Punzi, ${ }^{31}$ K. Ragan, ${ }^{23}$ D. Reher, ${ }^{21}$ A. Ribon, ${ }^{29}$ F. Rimondi, ${ }^{3}$ L. Ristori, ${ }^{31}$ W. J. Robertson, ${ }^{9}$ A. Robinson, ${ }^{23}$ T. Rodrigo, ${ }^{6}$ S. Rolli, ${ }^{41}$ L. Rosenson, ${ }^{22}$ R. Roser, ${ }^{10}$ R. Rossin, ${ }^{29}$ W. K. Sakumoto, ${ }^{34}$ D. Saltzberg, ${ }^{5}$ A. Sansoni, ${ }^{12}$ L. Santi, ${ }^{39}$ H. Sato, ${ }^{40}$ P. Savard, ${ }^{23}$ P. Schlabach, ${ }^{10}$ E. E. Schmidt, ${ }^{10}$ M. P. Schmidt, ${ }^{44}$ M. Schmitt, ${ }^{14}$ L. Scodellaro, ${ }^{29}$ A. Scott, ${ }^{5}$ A. Scribano, ${ }^{31}$ S. Segler, ${ }^{10}$ S. Seidel, ${ }^{26}$ Y. Seiya, ${ }^{40}$ A. Semenov, ${ }^{8}$ F. Semeria, ${ }^{3}$ T. Shah, ${ }^{22}$ M. D. Shapiro, ${ }^{21}$ P. F. Shepard, ${ }^{32}$ T. Shibayama, ${ }^{40}$ M. Shimojima, ${ }^{40}$ M. Shochet, ${ }^{7}$ J. Siegrist, ${ }^{21}$ G. Signorelli, ${ }^{31}$ A. Sill, ${ }^{38}$ P. Sinervo, ${ }^{23}$ P. Singh, ${ }^{16}$ A. J. Slaughter, ${ }^{44}$ K. Sliwa, ${ }^{41}$ C. Smith, ${ }^{17}$ F. D. Snider, ${ }^{10}$ A. Solodsky, ${ }^{35}$ J. Spalding, ${ }^{10}$ T. Speer, ${ }^{13}$ P. Sphicas, ${ }^{22}$ F. Spinella, ${ }^{31}$ M. Spiropulu, ${ }^{14}$ L. Spiegel, ${ }^{10}$ L. Stanco, ${ }^{29}$ J. Steele, ${ }^{43}$ A. Stefanini, ${ }^{31}$ J. Strologas, ${ }^{16}$ F. Strumia, ${ }^{13}$ D. Stuart, ${ }^{10}$ K. Sumorok, ${ }^{22}$ T. Suzuki, ${ }^{40}$ R. Takashima, ${ }^{15}$ K. Takikawa, ${ }^{40}$ M. Tanaka, ${ }^{40}$ T. Takano, ${ }^{28}$ B. Tannenbaum, ${ }^{5}$ W. Taylor, ${ }^{23}$ M. Tecchio, ${ }^{24}$ P. K. Teng, ${ }^{1}$ K. Terashi, ${ }^{40}$ S. Tether, ${ }^{22}$ D. Theriot, ${ }^{10} \mathrm{R}$. Thurman-Keup, ${ }^{2}$ P. Tipton, ${ }^{34} \mathrm{~S}$. Tkaczyk, ${ }^{10} \mathrm{~K}$. Tollefson, ${ }^{34}$ A. Tollestrup, ${ }^{10}$ H. Toyoda, ${ }^{28}$ W. Trischuk, ${ }^{23}$ J. F. de Troconiz, ${ }^{14}$ S. Truitt, ${ }^{24}$ J. Tseng, ${ }^{22}$ N. Turini, ${ }^{31}$ F. Ukegawa, ${ }^{10} \mathrm{~J}$. Valls, ${ }^{36} \mathrm{~S}$. Vejcik III, ${ }^{10} \mathrm{G}$. Velev, ${ }^{31} \mathrm{R}$. Vidal, ${ }^{10} \mathrm{R}$. Vilar, ${ }^{6} \mathrm{I}$. Vologouev, ${ }^{21}$ D. Vucinic, ${ }^{22}$ R. G. Wagner, ${ }^{2}$ R. L. Wagner, ${ }^{10}$ J. Wahl, ${ }^{7}$ N. B. Wallace, ${ }^{36}$ A. M. Walsh, ${ }^{36}$ C. Wang, ${ }^{9}$ C. H. Wang, ${ }^{1}$ M. J. Wang, ${ }^{1}$ T. Watanabe, ${ }^{40}$ T. Watts, ${ }^{36}$ R. Webb, ${ }^{37}$ H. Wenzel, ${ }^{18}$ W. C. Wester III, ${ }^{10}$ A. B. Wicklund, ${ }^{2}$ E. Wicklund, ${ }^{10}$ H. H. Williams, ${ }^{30}$ P. Wilson, ${ }^{10}$ B. L. Winer, ${ }^{27}$ D. Winn, ${ }^{24}$ S. Wolbers, ${ }^{10}$ D. Wolinski, ${ }^{24}$ J. Wolinski, ${ }^{25}$ S. Worm, ${ }^{26} \mathrm{X} . \mathrm{Wu},{ }^{13}$ J. Wyss, ${ }^{31}$ A. Yagil, ${ }^{10}$ W. Yao, ${ }^{21}$ G. P. Yeh, ${ }^{10}$ P. Yeh, ${ }^{1}$ J. Yoh, ${ }^{10}$ C. Yosef, ${ }^{25}$ T. Yoshida, ${ }^{28}$ I. Yu, ${ }^{19}$ S. Yu, ${ }^{30}$ A. Zanetti, ${ }^{39}$ F. Zetti, ${ }^{21}$ and S. Zucchelli ${ }^{3}$ 
(CDF Collaboration)

1 Institule of Physics, Academia Sinica, Taipei, Taiven 11529, Republic of China

2 Argonne National Laboretory, Argonne, Mllinois 60439

3 latitulo Narionale di Finica Nucleare, University of Bologna, I-10127 Bologna, Italy

4 Brandeis Universily, Walham, Massachwells 02254

5 University of California at Los Angeles, Los Angeles, California 90024

6 Institzlo de Fisica de Cantabria, Universily of Cantabria, sgoos Sanlander, Spain

7 Enrico Fermi Institule, Universily of Chicago, Chicago, Ilinois 60657

8 Joint Institute for Nwclear Research, RU-141980 Dubra, Rwesia

9 Duke University, Durham, North Caroline 27708

10 Fermi National Accelerator Laboratory, Batavia, Illinois 60510

11 Universily of Florida, Gainesville, Florida s2611

12 Laboratori Nazionali di Frascali, Istitulo Naxionale di Fisica Nucleare, I-00044 Frascali, Italy

13 Univeroily of Geneva, CH-1211 Geneve 4, Swilzerland

14 Harvard University, Cambridge, Massachwetle 02158

15 Hiroshima University, Higashi-Hiroshima 7eh, Japan

16 Univeraily of Illinois, Urbana, Illinois 61801

17 The Johns Hoptins Universily, Baltimore, Marvland 21218

18 Instilul fïr Experimentelle Kemphysit, Universilät Kerloruhe, 76128 Karlorahe, Germany

19 Korean Hadron Collider Laboratory: Krangpook National Universily, Teegn 702-701; Seoul National Universily, Seoul 151-742; and SungKyunkwan University, Swwon 140-746; Korea

20 High Energs Accelerelor Research Organization (KEK), Toutuba, Marati SO5, Japan

21 Ermest Orlando Lewrence Berteley National Laboratory, Berteley, Califormia 94780

22 Massachwells Inotitule of Technologn, Cambridge, Massachwetls 02159

23 Instilule of Particle Physics: McGill University, Montreal HSA $2 T 8$; and University of Toronto, Toronto MsS 1A7; Carada

24 Universily of Michigan, Ann Arbor, Michigen $\$ 8109$

25 Michigan Stete University, East Lansing, Michigan $\$ 8824$ 
27 The Ohio State Universily, Colamber, Ohio $\$ 9210$ 28

Osata Cily University, Osate 588, Jopan

29

Universila di Padova, Istitulo Naxionale di Fisica Nucleare, Serione di Padova, I-s5191 Padova, Italv

30

Univeraily of Pennoylvaria, Philadelphia, Penrovleania 19104

31 Istitulo Nazionale di Fivica Nucleare, University and Scwola Nomale Superiore of Pisa, I-56100 Pisa, Italy

32 Universily of Pillsburgh, Pilloburgh, Pensevlvanse 15260

33 Purdue University, West Lafayette, Indiana 47907

34 Universily of Rochester, Rochester, New Yort 14627

35 Rockefeller Univeraily, New York, New Yort 10021

36 Rulgers Universily, Piscalavey, New Jersey 08855

37 Tezas A $6 M$ University, College Station, Texas 77849

38 Tezas Tech Urizersity, Labbock, Tezas 79409

39 Istituto Narionale di Fivica Nucleare, Universily of Trieste/ Udine, Ilaly

40 Univeroity of Tsutwba, Tsukuba, Ibarati s05, Japan

41 Twfts University, Medford, Massachwetls 02155

42 Waseda University, Totyo 169, Japan

43 University of Wisconsin, Madison, Wisconsin 59706

44 Yale University, New Haven, Connecticut 06520 


\section{Bibliography}

[1] T. Affolder et al, Phys. Rev. Lett., 84, 216 (2000).

[2] F. Abe et ah, Phys. Rev. Lett., 74, 2626 (1995).

[3] F. Abachi et al, Phys. Rev. Lett., 74, 2632 (1995).

[4] The standard model is described in many references. Listed here is a brief sample of references that influenced the discussion in Chapter 1; V. Barger and R. Phillips, Collider Physics, Addison Wesley Publishing Company, (1987); G. Kane, Modern Elementary Particle Physics, Addison Wesley Publishing Company, (1993); M. Veltman, Diagrammatica: The Path to Feynman Diagrams, Cambridge University Press, (1994).

[5] G. Kane, C.-P. Yuan, and D. Ladinsky, Phys. Rev. D 45, 124, (1992).

[6] F. Abe et al., The CDF Detector: An Overview, Nucl. Inst. and Meth., A271, 387 (1988).

[7] H. Minemura et al., Nucl. Inst. and Methods A238, 18 (1985).

[8] D. Amidei et al, The CDF Silicon Vertex Detector, Nucl. Inst. and Meth., A350, 73 (1994).

[9] D. Amidei et al, SVX': The New CDF Silicon Vertex Detector. Nucl. Inst. and Meth., A360, 137, (1995).

[10] F. Snider et al., Nucl. Inst. and Meth., A268, 75 (1988). 
[11] F. Bedeschi et al., Nucl. Inst. and Meth., A268, 50 (1988).

[12] L. Balka et al., Nucl. Inst. and Meth., A267, 272 (1988).

[13] S. R. Hahn et al., Nucl. Inst. and Meth., A267, 351 (1988).

[14] K. Yasuoka et al., Nucl. Inst. and Meth., A267, 315 (1988).

[15] R. G. Wagner et al., Nucl. Inst. and Meth., A267, 330 (1988).

[16] T. Devlin et al., Nucl. Inst. and Meth., A268, 24 (1988).

[17] S. Bertolucci et al., Nucl. Inst. and Meth., A267, 301 (1988).

[18] Y. Fukui et al., Nucl. Inst. and Meth., A267, 280 (1988).

[19] W. C. Carithers et al., Proceedings of the Gas Sampling Calorimetry Workshop II, Batavia, Ilinois, 1985 (unpublished).

[20] G. Brandenburg et al., Nucl. Inst. and Meth., A267, 257 (1988).

[21] S Cihangir et al., Nucl. Inst. and Meth., A267, 249 (1988).

[22] M. Kruse, Observation of Top Quark Pair Production in the Dilepton Decay Channel from Proton-Antiproton Collisions at $\sqrt{s}=1.8 T e V$, Ph.D. thesis, Purdue University, 1996 (unpublished).

[23] F. Abe et al., Phys. Rev. Lett. 80, 2773 (1998).

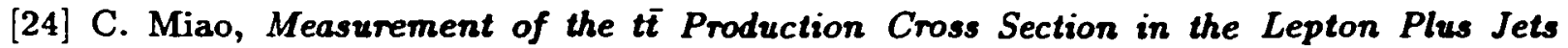
Channel in $\bar{p} p$ Collisions at $\sqrt{\mathrm{s}}=1.8 \mathrm{TeV}$, Ph.D. thesis, University of Michigan, 1997 (unpublished).

[25] D. Glenzinski, Observation of the Top Quark in Proton-Antiproton Collisions at a Center of Mass Energy of 1.8 TeV, Ph.D. thesis, The Johns Hopkins University, 1995. 
[26] F. Abe et al., Phys. Rev. Lett. 80, 2767 (1998). The CDF top mass analysis requires events to pass an additional "goodness of fit" cut based upon the kinematic fitter which is used to reconstruct the top mass. We use the efficiency of this cut to extrapolate the mass analysis backgrounds to the backgrounds required for this analysis, where no goodness of fit cut is applied.

[27] S. Aota et al., Measurement of the Top Quark Mass in the Lepton+Jets Channel, CDF internal Note 4199, 1997 (unpublished).

[28] A. Caner, CDFSIM + QFL Simulation of the CDF Detector, CDF internal note 2177 1993 (unpublished).

[29] M. Shapiro, A. Batti, J. Benloch, R. Harris, T. Rodrigo, P. Sphicas, T. Westhusing, $A$ User's Guide to QFL, CDF Internal Note 1810, 1991 (unpublished).

[30] J. Benlloch and T. Rodrigo, Tracking in QFL, CDF Internal note 1634, 1991 (unpublished).

[31] T. Hessing and B. Winer, A Brief Note Comparing Jet Data to QFL, CDF Internal note 1347,1991 , (unpublished).

[32] A. Roodman, Update on Central Calorimenter Response to Pions and Tuning of QFL, CDF Internal note 1344, 1991 (unpublished).

[33] R. Harris, S. Kuhlmann, M. Shapiro, and B. Wicklund, Simulation of CES Showers and $\chi^{2}$ in $Q F L$, CDF Internal note 1222, 1990 (unpublished).

[34] M. Shapiro, D. Brown, S. Kannappan, QFL Version 2.0: Improvements and Checks, CDF Internal note 753, 1988 (unpublished).

[35] G. Marchesini and B.R. Webber, Nucl. Phys. B 310, 461 (1988); G. Marchesini et al., Comput. Phys. Commun. 67, 465 (1992); We use a custom modification of the HERWIG program which was written by G. Guillian (unpublished). 
[36] T. Sjōstrand, Comput. Phys. Commun. 82, 74 (1994). We use PYTHIA version 5.7.

[37] F.A. Berends, W.T. Giele, H. Kuijf, and B. Tausk, Nucl. Phys. B 357, 32 (1991).

[38] G. Guillian, M. Campbell, D. Amidei, A Modified Version of HERWIG for Studying the Spin Properties of the Top Quark, CDF Internal note 4261, 1997 (unpublished).

[39] F. Abe et al., Phys. Rev. Lett. 73, 2296 (1994); F. Abe et al., Fermilab-Pub-98/327-E.

[40] T. Liss and R. Roser, $t \overline{\mathrm{t}}$ Production Cross Section for 110, CDF Internal note 3481, 1997 (unpublished).

[41] P. Bevington, Data Reduction and Error Analysis for the Physical Sciences McGrawHill, Inc. New York, 1969

[42] F. Abe et al., The CDF II Detector Technical Design Report, FERMILAB-Pub-96/390$\mathbf{E},(1996)$. 


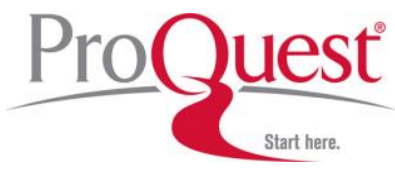

\section{ProQuest Distribution Agreement}

This Agreement is between the author (Author) and ProQuest LLC, through its ProQuest Dissertation Distribution business (formerly ProQuest/UMI). Under this Agreement, Author grants ProQuest certain rights to preserve, archive and distribute the dissertation or thesis (the Work), abstract, and index terms provided by Author to ProQuest.

\section{Section I. License for Inclusion of the Work in ProQuest Publishing Program}

Grant of Rights. Author hereby grants to ProQuest the non-exclusive, worldwide right to reproduce, distribute, display and transmit the Work (in whole or in part) in such tangible and electronic formats as may be in existence now or developed in the future. Author further grants to ProQuest the right to include the abstract, bibliography and other metadata in the ProQuest Dissertations \& Theses database (PQDT) and in ProQuest Dissertation Abstracts International and any successor or related index and/or finding products or services.

ProQuest Publishing Program - Election and Elements. The rights granted above shall be exercised according to the publishing option selected by Author in Section III, Author Options, and subject to the following additional Publishing Program requirements:

- Distribution of the Work. Except as restricted by Author in the publishing option selected, the rights granted by Author automatically include (1) the right to allow sale and distribution of the Work, in whole or in part, by ProQuest and its sales agents and distributors and (2) the right to make the Abstract, bibliographic data and any meta data associated with the Work available to search engines and harvesters.

- Restrictions. ProQuest will use commercially reasonable efforts to restrict the distribution of the Work as provided under the publishing option selected by Author or as later elected by Author through direct contact with ProQuest. Such election is subject to Author's Degree Granting Institution Directives. With respect to restrictions requested after submission of the Work, Author acknowledges that ProQuest cannot recall or amend previously distributed versions of the Work.

- Removal of Work from the Program. ProQuest may elect not to distribute the Work if it believes that all necessary rights of third parties have not been secured. Refer to the website http://www.proquest.com/products_umi/dissertations/for information about copyright and your dissertation or thesis. If Author's degree is rescinded, and/or the degree-granting institution so directs, ProQuest will expunge the Work from its publishing program in accordance with its then current publishing policies.

- $\quad$ Degree Granting Institution Directives. Author is solely responsible for any conflict between policies and directions of Author's degree-granting institution, Author's choice of publishing model, and/or any restriction Author places on the use of the Work. For the avoidance of doubt, ProQuest is not responsible for access to the Work that is provided by Author's degree-granting institution through its library or institutional repository. Author must work directly with Author's degree granting institution to ensure application of any restrictions to access relating to the Work by Author's degree granting institution.

Delivery of the Work. Author shall provide to ProQuest the Work and all necessary supporting documents during the online submission process, according to the Instructions accompanying this agreement.

Rights Verification. Author represents and warrants that Author is the copyright holder of the Work and has obtained all necessary rights to permit ProQuest to reproduce and distribute third party materials contained in any part of the Work, including all necessary licenses for any non-public, third party software necessary to access, display, and run or print the Work. Author is solely responsible and will indemnify ProQuest for any third party claims related to the Work as submitted for publication.

Open Access Publishing Plus

I want the broadest possible dissemination of my work, and I want to provide free global access to the electronic copy of my work via the internet.

$\square \quad$ I understand that I will not be eligible to receive royalties.

I want major search engines (e.g. Google, Yahoo) to discover my work. Learn more: http://www.proquest.com/en-

US/products/dissertations/google.shtml

$\sqrt{ }$ Yes

$\square \quad$ No

Acknowledgment: I have read, understand and agree to this ProQuest Publishing Formatted: Indent: Left: $0.75 "$, Bulleted Level: 2 + Aligned at: $0.81^{\prime \prime}+$ Indent at: 1.06

the publishing option chosen by me as indicated

(Print Name)_David Wing

Institution conferring degree Univessily of Micligan

Formatted: Indent: Left: $0.69 "$, First line: $0.07 "$, Bulleted + Level: 1 + Aligned at: $0.69^{\prime \prime}$ + Indent at: $0.78^{\prime \prime}$ 


\section{Dissertation/Master's Thesis Submission Form}

Please print clearly in block letters

\section{Personal Information}

Last Name $\frac{\text { Winn }}{\text { First Name }}$

_ M (Master's Thesis)

$\underline{\text { D }}$ (Dissertation)

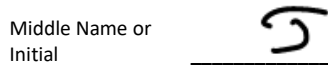

Country (ies) of Citizenship

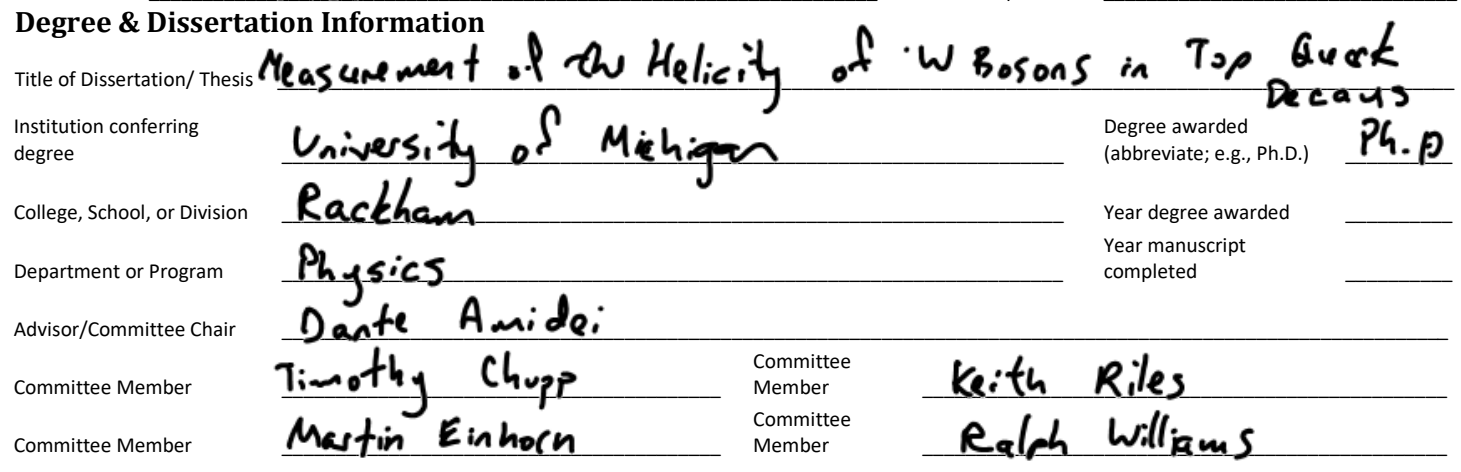




\section{English}

Primary Subject Category: Enter the 4-digit code and category name from the Subject Category Guide that most closely describes the area of your research.

You may suggest two additional subject categories that may aid in the discovery of your work in our digital database.

\section{code 0605 category Physies code__tegory \\ Provide up to 6 keywords or short phrases for citation indices, library cataloging, and database searching.}

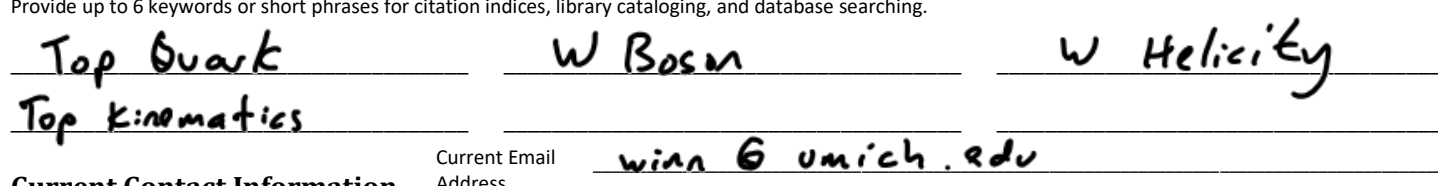

Current Contact Information Address

\section{Randall Lah // 500 East univerity}

Please provide your postal address if you are interested in receiving royalties on sales of your thesis.

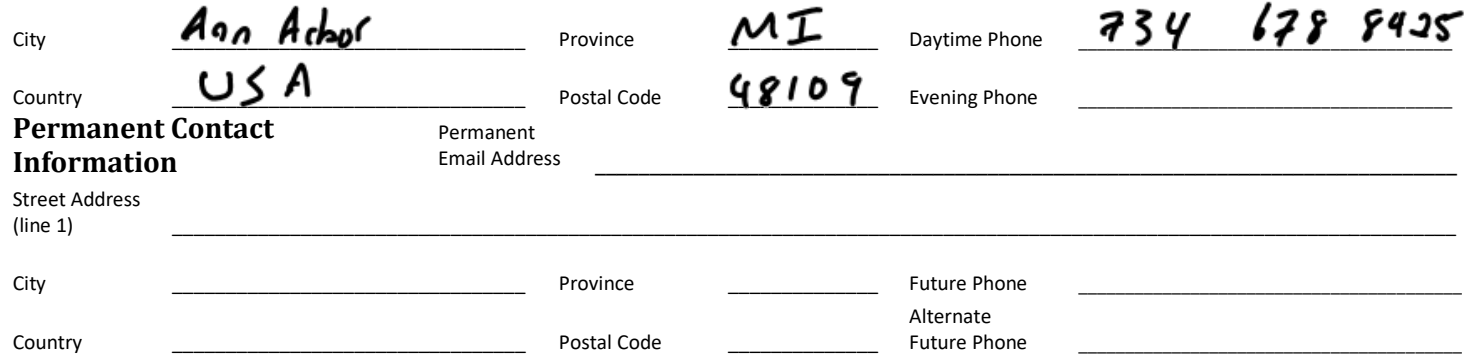

THIS PAGE MUST ACCOMPANY YOUR MANUSCRIPT AND THE REST OF YOUR SUBMISSION MATERIALS

Subject Categories

The ProQuest Dissertations \& Theses (PQDT) database and the ProQuest citation indices are arranged by subject categories. Please select the one category below that best describes your field of research or creative work. You may add one or two additional categories on your submission form that will also be associated with your work as secondary subjects.

\section{Arts, Business, Education, Humanities, and Social Sciences}

\begin{tabular}{ll} 
AREA, ETHNIC, AND GENDER \\
\hline STUDIES \\
\hline African American studies & 0296 \\
African studies & 0293 \\
American studies & 0323 \\
Asian American studies & 0343 \\
Asian studies & 0342 \\
Baltic studies & 0361 \\
Black studies & 0325 \\
Canadian studies & 0385 \\
Caribbean studies & 0432 \\
Classical studies & 0434 \\
East European studies & 0437 \\
Ethnic studies & 0631 \\
European studies & 0440 \\
French Canadian culture & 0482 \\
Gender studies & 0733 \\
GLBT studies & 0492 \\
Hispanic American studies & 0737
\end{tabular}

$\begin{array}{lllr}\text { Holocaust studies } & 0507 & & \\ \text { Islamic culture } & 0512 & \text { BUSINESS } & \\ \text { Judaic studies } & 0751 & \text { Accounting } & 0272 \\ \text { Latin American studies } & 0550 & \text { Arts management } & 0424 \\ \text { Middle Eastern studies } & 0555 & \text { Banking } & 0770 \\ \text { Native American studies } & 0740 & \text { Business } & 0310 \\ \text { Near Eastern studies } & 0559 & \text { Entrepreneurship } & 0429 \\ \text { North African studies } & 0560 & \text { Finance } & 0508 \\ \text { Pacific Rim studies } & 0561 & \text { Management } & 0454 \\ \text { Regional studies } & 0604 & \text { Marketing } & 0338 \\ \text { Scandinavian studies } & 0613 & \text { Sports management } & 0430 \\ \text { Slavic studies } & 0614 & \text { COMMUNICATIONS AND } & \\ \text { South African studies } & 0654 & \text { INFORMATION SCIENCES } & \\ \text { South Asian studies } & 0638 & \text { Communication } & 0459 \\ \text { Sub Saharan Africa studies } & 0639 & \text { Information science } & 0723 \\ \text { Women's studies } & 0453 & \text { Journalism } & 0391 \\ & & \text { Library science } & 0399 \\ & & \text { Mass communication } & 0708 \\ & & \text { Technical communication } & 0643\end{array}$


FINE AND PERFORMING ARTS

\section{Art criticism}

Art history

Cinematography

Dance

Design

Film studies

Fine arts

Music

Performing arts

Theater

Theater history

\section{EDUCATION}

Adult education

Art education

Bilingual education

Business education

Community college education

Continuing education 0651

Curriculum development $\quad 0727$

Early childhood education 0518

Education 0515

Education finance

Education policy

Educational administration

Educational evaluation $\quad 0443$

Educational leadership $\quad 0449$

Educational psychology 0525

Educational technology 0710

Educational tests \& measurements 0288

Elementary education 0524

English as a second language $\quad 0441$

Foreign language instruction $\quad 0444$

Gifted education

Health education

Higher education

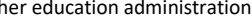

History of education

Home economics education

Industrial arts education

Instructional design

Language arts

Mathematics education

Middle school education

Multicultural education

Music education

Pedagogy

Performing arts education

Philosophy of education

Physical education

Reading instruction

Religious education

School counseling

Science education

Secondary education

Social sciences education

Sociology of education

Special education

Teacher education

Vocational education
0516

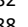

(1)

(2)

4

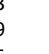

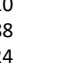

\section{HUMANITIES}

\begin{tabular}{ll} 
HISTORY & \\
\hline African history & 0331 \\
American history & 0337 \\
Ancient history & 0579 \\
Asian history & 0332 \\
Black history & 0328 \\
Canadian history & 0334 \\
European history & 0335 \\
History & 0578 \\
History of Oceania & 0504 \\
History of science & 0585 \\
Latin American history & 0336 \\
Medieval history & 0581 \\
Middle Eastern history & 0333 \\
Military history & 0772 \\
Modern history & 0582 \\
Russian history & 0724 \\
World history & 0506
\end{tabular}

LANGUAGE \& LITERATURE African literature 0316 American literature 0591 Ancient languages $\quad 0289$ Asian literature 0305 British and Irish literature $\quad 0593$ Canadian literature $\quad 0352$ Caribbean literature 0360 Classical literature $\quad 0294$ Comparative literature $\quad 0295$ English literature 0593 French Canadian literature $\quad 0355$

Germanic literature 0311 Icelandic \& Scandinavian literature 0362 Language 0679 Latin American literature $\quad 0312$ Linguistics $\quad 0290$

Literature 0401

Literature of Oceania $\quad 0356$

Medieval literature $\quad 0297$

Middle Eastern literature $\quad 0315$

Modern language $\quad 0291$

Modern literature $\quad 0298$

Rhetoric 0681

Romance literature $\quad 0313$

Slavic literature $\quad 0314$

PHILOSOPHY AND RELIGION

$\begin{array}{ll}\text { Aesthetics } & 0650 \\ \text { Biblical studies } & 0321 \\ \text { Canon law } & 0375 \\ \text { Clerical studies } & 0319 \\ \text { Comparative religion } & 0618 \\ \text { Divinity } & 0376 \\ \text { Epistemology } & 0393 \\ \text { Ethics } & 0394 \\ \text { Logic } & 0395 \\ \text { Metaphysics } & 0396 \\ \text { Pastoral counseling } & 0397 \\ \text { Philosophy } & 0422 \\ \text { Philosophy of Religion } & 0322 \\ \text { Philosophy of science } & 0402 \\ \text { Religion } & 0318 \\ \text { Religious history } & 0320 \\ \text { Spirituality } & 0647 \\ \text { Theology } & 0469\end{array}$




\section{LAW AND LEGAL STUDIES}

dispute resolution

Intellectual property 0513

International law 0616

Patent law

\section{SOCIAL SCIENCES}

Archaeology

032

Criminology

Cultural anthropology

Demography

Economic history

Economic theory

Economics

Economics, Commerce-Business 0505

Economics, Labor

Folklore

Forensic anthropology

Geography

International relations $\quad 0601$

Military studies

Organization theory

Organizational behavior

Peace studies

Physical anthropology

Political Science

Public administration

Public policy

Recreation and tourism

Social research

Social structure

Social work

Sociolinguistics

Sociology

Transportation planning

Urban planning

\section{INTERDISCIPLINARY}

Alternative energy 0363

Biographies 0304

Climate change $\quad 0404$

Cultural resources management 0436

Energy 0791

Food science 0359

Home economics 0386

Information technology 0489

Multimedia $\quad 0558$

Museum studies $\quad 0730$

Sustainability 0640

Textile research 0994

Wood sciences $\quad 0746$

\section{Behavioral, Natural, and Physical Sciences}

\section{AGRICULTURE}

Agriculture

Agronomy

Animal diseases

Animal sciences

Fisheries and aquatic sciences

Forestry

Horticulture

Plant pathology

Plant sciences

Range management

Soil sciences

Urban forestry

Wildlife management

0473
0285
0476
0475
0792
0478
0471
0480
0479
0777
0481
0281
0286

Behavioral sciences

Clinical psychology

Cognitive psychology

Counseling psychology

Developmental psychology

Experimental psychology

Occupational psychology

Personality psychology

Physiological psychology

Psychobiology

Psychology

Quantitative psychology and

psychometrics

Social psychology

\section{ARCHITECTURE}

\section{Architecture}

Architectural engineering

Landscape architecture

0729
0462

0390

BEHAVIORAL SCIENCES

Animal behavior

\section{BIOLOGICAL SCIENCES}

Biochemistry

Bioinformatics

Biology

Biomechanics

Biophysics

Biostatistics
Cellular biology

Developmental biology

Endocrinology

Entomology

Genetics

Histology

Limnology

Microbiology

Molecular biology

Morphology

Neurosciences

Parasitology

Physiology

Plant biology

Systematic biology

Virology

Zoology 


\section{ECOSYSTEM SCIENCES}

\section{Ecology}

Macroecology

Paleoecology

0329

0420

ENGINEERING

\section{Aerospace engineering}

Artificial intelligence

Automotive engineering

Biomedical engineering

Chemical engineering

Civil engineering

Computer engineering

Computer science

Electrical engineering

Engineering

Geological engineering

Geophysical engineering

Geotechnology

Industrial engineering

Mechanical engineering

Mining engineering

Naval engineering

Nanotechnology

Nuclear engineering

Ocean engineering

Operations research

Packaging

Petroleum engineering

Plastics

Robotics

System science

0538

0800

0540

0541

0542

0543

0464

0984

0544

0537

0466

0467

0428

0546

0548

0551

0468

0652

0552

0547

0796

0549

0765

0795

0790

\section{ENVIRONMENTAL SCIENCES}

\section{Conservation biology}

Environmental economics

Environmental education

Environmental engineering

Environmental geology

Environmental health

Environmental justice

Environmental law

Environmental management

Environmental philosophy

Environmental science

Environmental studies

Land use planning

Natural resource management

Water resources management

Wildlife conservation

0408

0438

0442

0775

0407

0470

0619

0439

0474

0392

0768

0477

0536

0528

0595

0284

MATHEMATICAL AND

\section{PHYSICAL SCIENCES}

Acoustics

Analytical chemistry

Applied mathematics

Astronomy 


$\begin{array}{ll}\text { Astrophysics } & 0596 \\ \text { Atomic physics } & 0748 \\ \text { Chemistry } & 0485 \\ \text { Condensed matter physics } & 0611 \\ \text { Electromagnetics } & 0607 \\ \text { High temperature physics } & 0597 \\ \text { Inorganic chemistry } & 0488 \\ \text { Low temperature physics } & 0598 \\ \text { Materials science } & 0794 \\ \text { Mathematics } & 0405 \\ \text { Mechanics } & 0346 \\ \text { Molecular chemistry } & 0431 \\ \text { Molecular physics } & 0609 \\ \text { Nanoscience } & 0565 \\ \text { Nuclear chemistry } & 0738 \\ \text { Nuclear physics } & 0756 \\ \text { Optics } & 0752 \\ \text { Organic chemistry } & 0490 \\ \text { Particle physics } & 0798 \\ \text { Physical chemistry } & 0494 \\ \text { Physics } & 0605 \\ \text { Plasma physics } & 0759 \\ \text { Polymer chemistry } & 0495 \\ \text { Quantum physics } & 0599 \\ \text { Statistics } & 0463 \\ \text { Theoretical mathematics } & 0642 \\ \text { Theoretical physics } & 0753 \\ & \end{array}$

